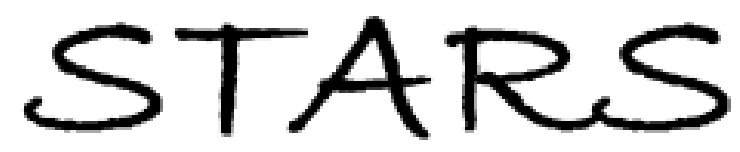

University of Central Florida

STARS

2012

\title{
Engineering Evaluation Of Multi-beam Satellite Antenna Boresight Pointing Using Land/water Crossings
}

Catherine Susan May

University of Central Florida

Part of the Electrical and Electronics Commons

Find similar works at: https://stars.library.ucf.edu/etd

University of Central Florida Libraries http://library.ucf.edu

This Masters Thesis (Open Access) is brought to you for free and open access by STARS. It has been accepted for inclusion in Electronic Theses and Dissertations, 2004-2019 by an authorized administrator of STARS. For more information, please contact STARS@ucf.edu.

\section{STARS Citation}

May, Catherine Susan, "Engineering Evaluation Of Multi-beam Satellite Antenna Boresight Pointing Using Land/water Crossings" (2012). Electronic Theses and Dissertations, 2004-2019. 2154.

https://stars.library.ucf.edu/etd/2154

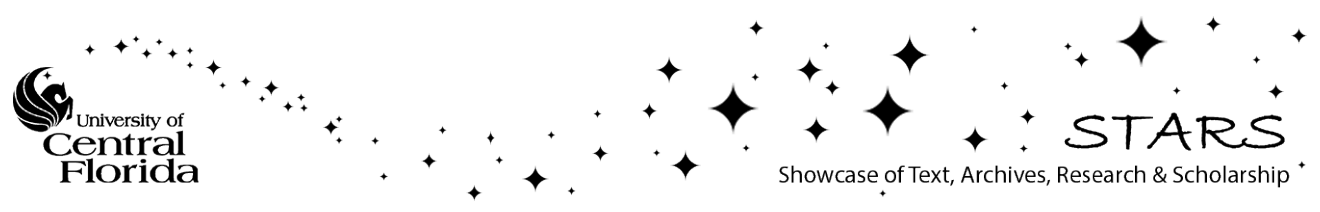




\title{
ENGINEERING EVALUATION OF MULTI-BEAM SATELLITE ANTENNA BORESIGHT POINTING USING LAND/WATER CROSSINGS
}

\author{
by
}

\section{CATHERINE SUSAN MAY}

B.S. University of Nebraska Lincoln

\begin{abstract}
A thesis submitted in partial fulfillment of the requirements for the degree of Master of Science in the School of Electrical Engineering and Computer Science in the College of Engineering and Computer Science at the University of Central Florida Orlando, Florida
\end{abstract}

Spring Term

2012

Major Professor: W. Linwood Jones 


\begin{abstract}
The Microwave Radiometer (MWR) on the Aquarius/SAC-D mission measures microwave radiation from earth and intervening atmosphere in terms of brightness temperature (Tb). It takes measurements in a push-broom fashion at $\mathrm{K}(23.8 \mathrm{GHz})$ and $\mathrm{Ka}(36.5 \mathrm{GHz})$ band frequencies using two separate antenna systems, each producing eight antenna beams. Pre-launch knowledge of the alignment of these beams with respect to the space-craft is used to geolocate the antenna footprints on ground. As a part of MWR's on-orbit engineering check-out, the verification of MWR's pointing accuracy is discussed here. The technique used to assess MWR's pointing involved comparing the radiometer image of land with high-resolution maps. When the beam's instantaneous field of view (IFOV) passes over a land water boundary, the brightness temperature changes from a radiometrically hot land scene to a radiometrically cold ocean scene. This "step-function" change in brightness temperature provides a very sensitive way to characterize the mispointing error of the MWR sensor antenna footprints. This thesis describes the algorithm used for the MWR geolocation calibration. MWR sensor observed boundaries are determined by the absolute maximum $\mathrm{Tb}$ slope location. A system of linear equations is produced for each sensor observed land/water crossing to determine the true intersection of the MWR track with the coastline. The observed and expected boundary locations are compared by means of an error distance. Results, presented for all eight beams of the three MWR channels, show that the mispointing error (standard deviations) are overall less than $15 \mathrm{~km}$ from the true coastline.
\end{abstract}




\section{ACKNOWLEDGEMENT}

First, and foremost, I would like to thank Dr. W. Linwood Jones. By accepting me in to the Central Florida Remote Sensing Lab, he allowed me to pursue a degree, and ultimately a career, in a field which perfectly encompasses my vast interests. Without his effort, time and advice, I would not have been able to complete this master's program in two, short years. I will always carry with me the many things that I have learned from him. He is truly an inspirational, motivating person, and for that I am forever grateful.

I would also like to thank my committee members, Dr. Wasfy Mikhael and Dr. Parveen Wahid. Thank you to all the members of the Central Florida Remote Sensing Lab for your support throughout the last two years, specifically Spencer Farrar for his assistance in this thesis project. I thoroughly enjoyed every minute with all of you. We have made many memories, and a few recipes, that I will never forget.

My family and friends, thank you for the continued support throughout my entire college career. Each one of you helps me in a unique, important way. You inspire me, you keep me sane, you listen, you love, you laugh, but most importantly you are always there for me. Specifically, I want to thank my parents. I am so proud to call myself your daughter. You have instilled in me great values, a drive to succeed and given me a support system that is never wavering. I am truly blessed to have so many amazing people in my life!

Thank you to the SMART Scholarship Program for funding my graduate education. This work was also supported under a NASA Headquarters grant for the NASA Ocean Surface Salinity Team (Aquarius Program). I wish to acknowledge the support of the entire CONAE MWR team and a special thanks to Juan-Cruz Gallo and Daniel Rocca. 


\section{TABLE OF CONTENTS}

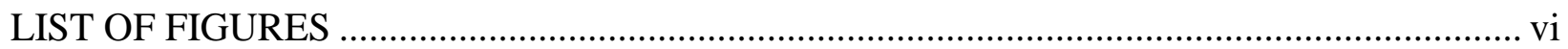

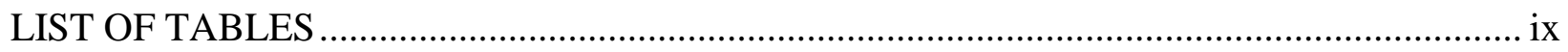

LIST OF ACRONYMS/ABBREVIATIONS ..................................................................

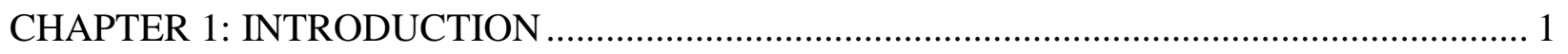

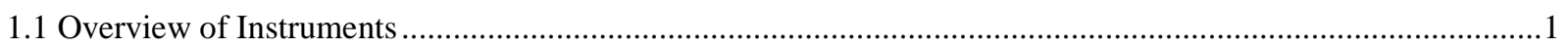

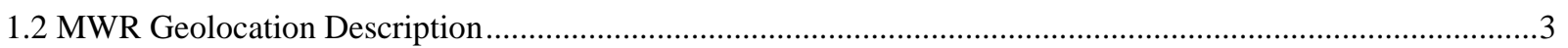

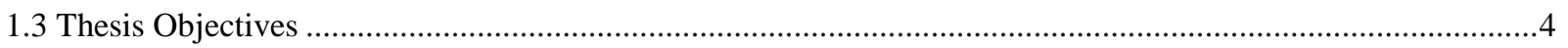

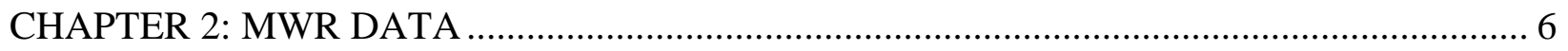

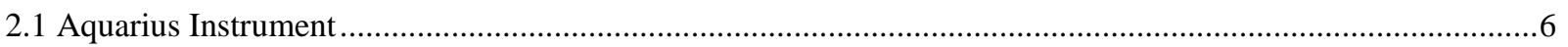

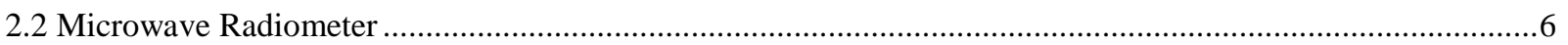

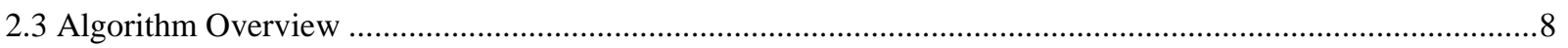

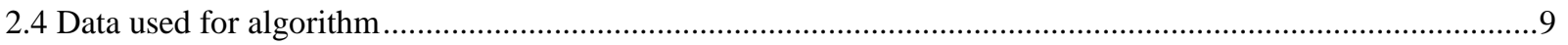

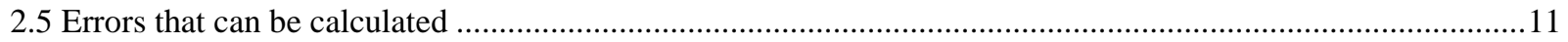

2.5.1 Antenna Pattern Error .......................................................................................................... 11

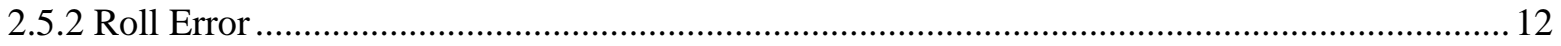

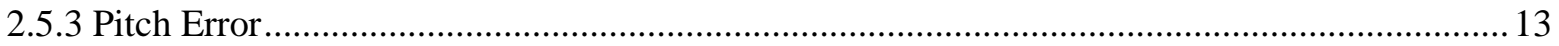

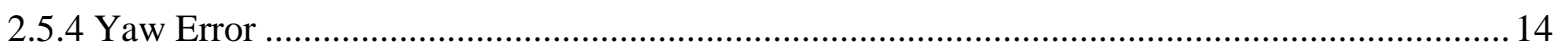

CHAPTER 3: GEOLOCATION ASSESSMENT ALGORITHM ......................................... 15

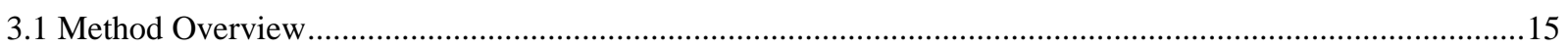

3.2 Experimental Observation of Land/Water Boundary ..............................................................................15

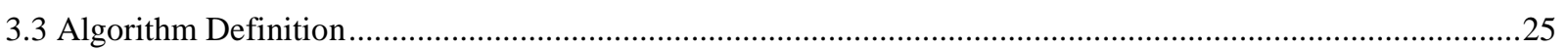

3.3.1 Tb Slope Filter (First three Blocks) ….......................................................................... 28

3.3.2 Assigning Latitude and Longitude to the parabolic maximum slope ...................................... 35

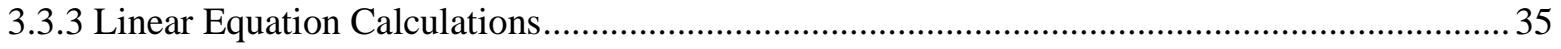

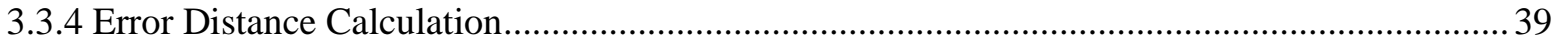

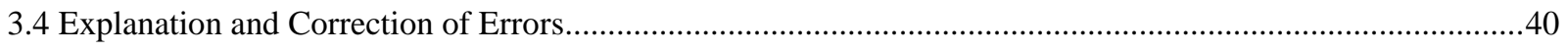

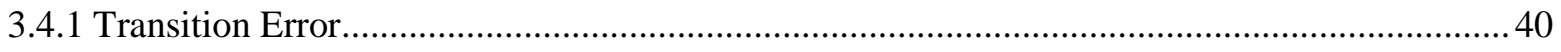

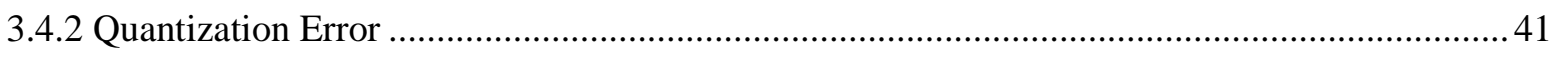




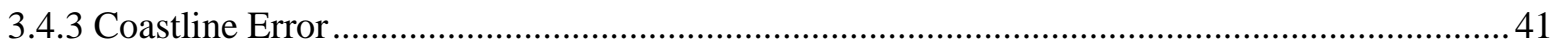

CHAPTER 4: RESULTS AND VALIDATION ................................................................... 43

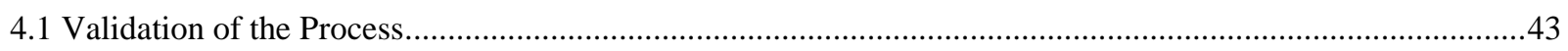

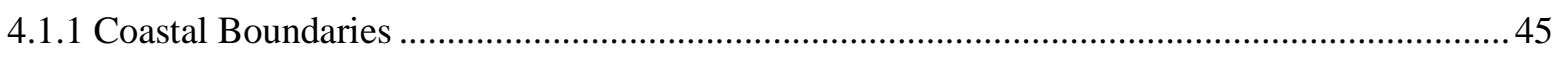

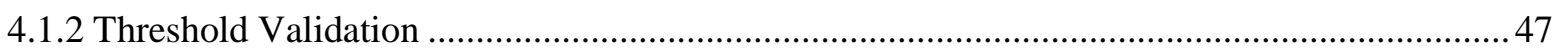

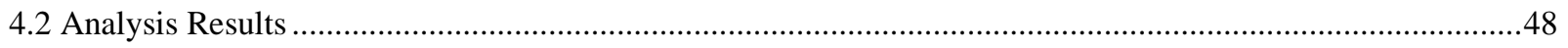

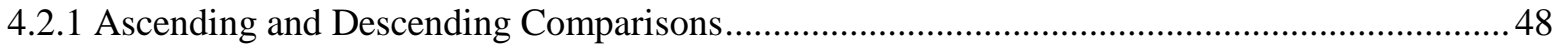

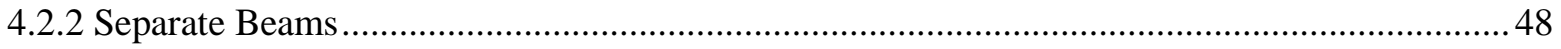

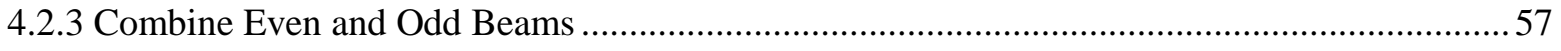

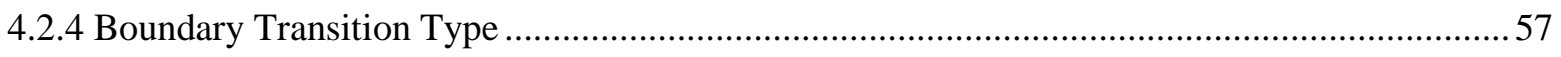

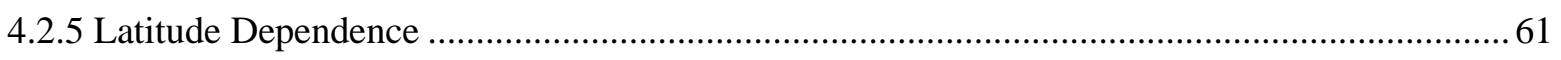

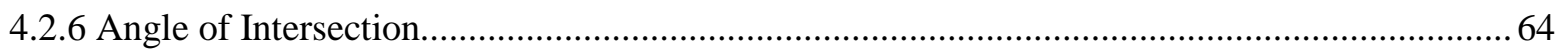

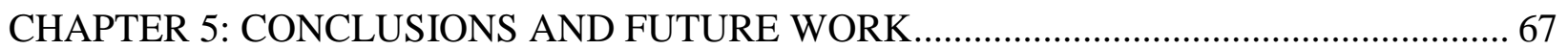

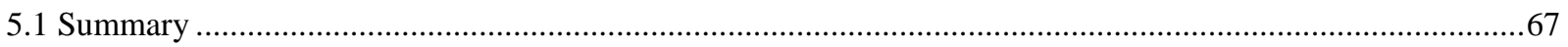

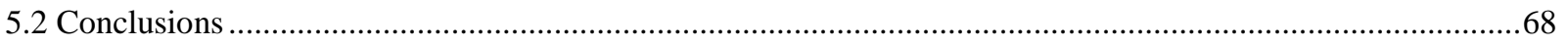

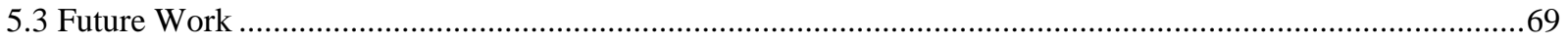

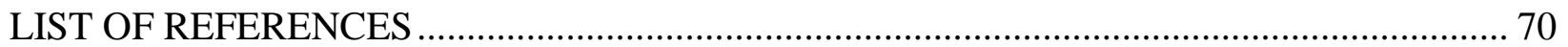




\section{LIST OF FIGURES}

Figure 1-1 Aquarius and MWR measurement geometry ................................................ 2

Figure 2-1 Image of MWR feed horns and reflectors ......................................................... 7

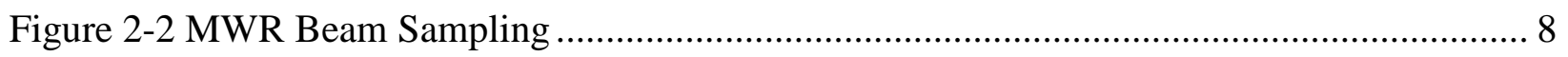

Figure 2-3 All ascending passes used for geolocation assessment (Nov. 14-20, 2011)............. 10

Figure 2-4 All Descending passes used for geolocation assessment (Nov. 14-20, 2011)........... 10

Figure 2-5 Negative roll causes the beam to be displaced to the right on Earth's surface ........... 12

Figure 2-6 Positive pitch means the front is angled higher than the rear of the spacecraft .......... 13

Figure 2-7 Yaw error causes the forward and aft beams to cross the boundary at different

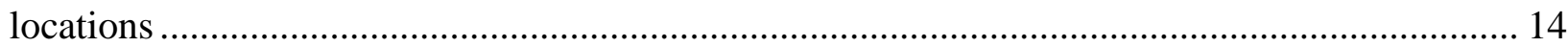

Figure 3-1 MWR track, all eight beams, approximately 20 orbits. The ocean is radiometrically 'cold', and the land is 'hot'.

Figure 3-2 Simulated brightness temperatures: upper panel is the theoretical apparent Tb for an ocean/land transition and lower panel is the simulated MWR antenna temperature................... 18

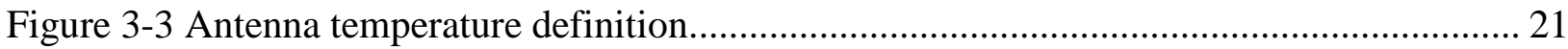

Figure 3-4 MWR 37-H GHz, beam-3 relative antenna pattern ............................................... 23

Figure 3-5 MWR 37-H GHz, beam-3, relative directivity pattern (blue), Gaussian pattern (red) 24

Figure 3-6 Simulated MWR antenna temperature (blue) from Gaussian antenna pattern convolution with step-function surface Tb for an ocean/land crossing. Also shown is the corresponding slope of the convolved brightness temperature (green). .................................... 24

Figure 3-7 Geolocation assessment algorithm block diagram ............................................... 27

Figure 3-8 Beam 3, 37V IFOVs across a coastal boundary ................................................... 29

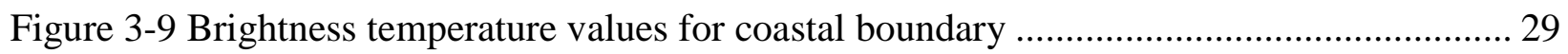

Figure 3-10 Slope threshold applied to a 37V coastal crossing ............................................. 30

Figure 3-11 One dimensional convolution of antenna pattern with land/water Tb step function.

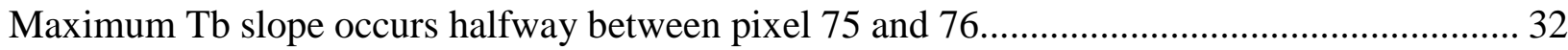

Figure 3-12 Parabolic fit to determine absolute Tb slope maximum...................................... 33

Figure 3-13 Comparison of MWR and parabolic fit, land to water maximum slope (red circleMWR observed boundary, blue triangle - parabolic max)..................................................... 34

Figure 3-14 MWR track linear equation representation ...................................................... 36 
Figure 3-15 Coastline approximation of coastal points within a +/- 0.1 degree box.................. 37

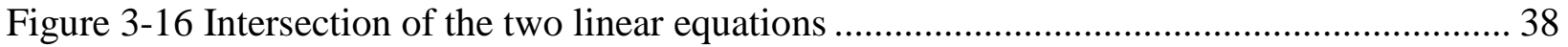

Figure 4-1 Slope value for MWR pixels (absolute maximum indicated as open circle)............. 44

Figure 4-2 Linear equations and intersection point (error distance is calculated from open circle

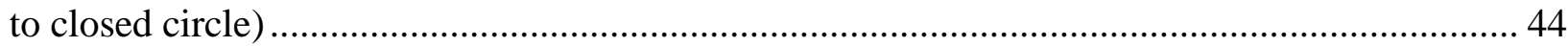

Figure 4-3 MWR sensor observed boundaries and Matlab generated coast (blue-descending,

red-ascending) with beam-4 IFOVs shown for an ascending orbit 46

Figure 4-4 MWR sensor observed boundaries and 1 km resolution coast (blue-descending, red-

ascending) with beam-4 IFOVs shown for an ascending orbit.............................................. 46

Figure 4-5 Beam 1, 23H channel, descending error distance .............................................. 50

Figure 4-6 Beam 2, 23H channel, descending error distance .............................................. 50

Figure 4-7 Beam 3, 23H channel, descending error distance .............................................. 51

Figure 4-8 Beam 4, 23H channel, descending error distance ............................................. 51

Figure 4-9 Beam 5, 23H channel, descending error distance ............................................... 52

Figure 4-10 Beam 6, 23H channel, descending error distance ............................................... 52

Figure 4-11 Beam 7, 23H channel, descending error distance .............................................. 53

Figure 4-12 Beam 8, 23H channel, descending error distance .............................................. 53

Figure 4-13 Original mean and standard deviation error bars, 23H ...................................... 54

Figure 4-14 Parabolic fit mean and standard deviation error bars, 23H................................. 54

Figure 4-15 Original mean and standard deviation error bars, 37V ....................................... 55

Figure 4-16 Parabolic fit mean and standard deviation error bars, 37V ................................... 55

Figure 4-17 Original mean and standard deviation error bars, 37H ....................................... 56

Figure 4-18 Parabolic fit mean and standard deviation error bars, 37H ................................. 56

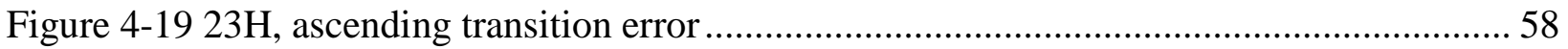

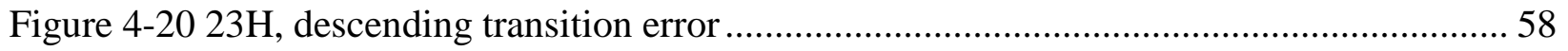

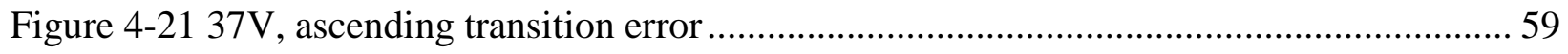

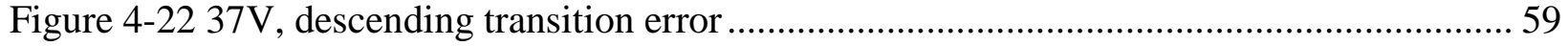

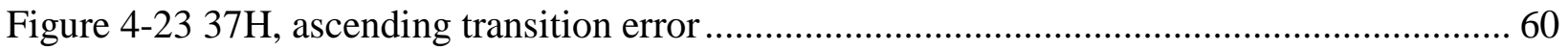

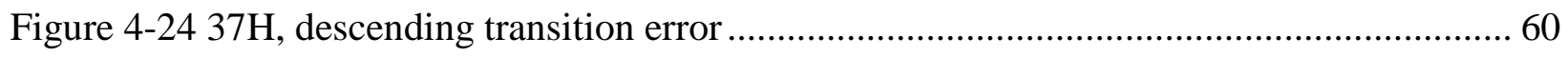

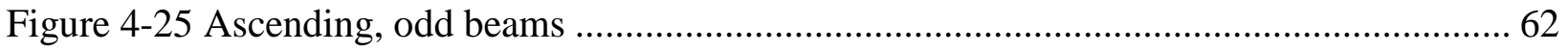




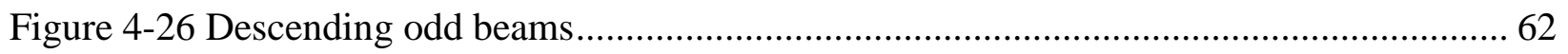

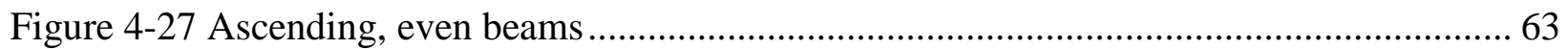

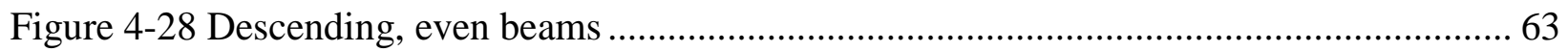

Figure 4-29 Angle of intersection used to determine attitude errors: perpendicular and parallel. 65 Figure 4-30 Histogram of MWR and coastline angle of intersection for even beams, 23H........ 66

Figure 4-31 Mean and standard deviation error distance by angle of intersection ..................... 66 


\section{LIST OF TABLES}

Table 1 Number of boundaries used to estimate the error distance statistics for $23 \mathrm{H}$................ 47

Table 2 Number of boundaries used to estimate the error distance statistics for 37V ................ 47

Table 3 Number of boundaries used to estimate the error distance statistics for $37 \mathrm{H} . . . \ldots \ldots \ldots \ldots . . . .47$ 


\section{LIST OF ACRONYMS/ABBREVIATIONS}

CFRSL Central Florida Remote Sensing Lab

CONAE Comisión Nacional de Actividades Espaciales (Argentine Space Agency)

EIA Earth Incidence Angle

IFOV Instantaneous Field of View

MWR Microwave Radiometer

NASA National Aeronautics and Space Administration

Tb Brightness Temperature 


\section{CHAPTER 1：INTRODUCTION}

Aquarius/SAC-D is a mission of discovery developed jointly by National Aeronautics and Space Administration (NASA) and the Comisión Nacional de Actividades Espaciales (Argentine Space Agency, CONAE) [1]. This Earth Science Program was launched June 10, 2011 providing global sea surface salinity (SSS) measurements to advance knowledge of the Earth’s hydrological cycle and its impact on global climate change. Aquarius (AQ) measurements will provide scientists with unprecedented global SSS measurements with high spatial (150 km) and temporal (7 days) sampling. In fact, within the first month after commissioning, Aquarius has provided more measurements than the entire SSS historical data record, previously provided by oceanographic ship and buoy observations.

For Aquarius/SAC-D, NASA served as the mission project management, developed the main instrument, Aquarius, and provided the launch from Vandenburg, CA. For its part, CONAE provided the satellite platform (SAC-D) and several instruments, which includes the Microwave Radiometer (MWR). The Central Florida Remote Sensing Lab (CFRSL) has collaborated with CONAE on the MWR instrument by conducting the on-orbit radiometric (brightness temperature, Tb) calibration [2][3] and geolocation validation, the latter of which is the subject of this thesis.

\section{$\underline{1.1 \text { Overview of Instruments }}$}

Aquarius/SAC-D is a sun-synchronous polar orbiting satellite with the sensors' beams pointing away from the sun to prevent intercepted solar radiation contamination as shown in Fig. 1-1. The Aquarius radiometer/scatterometer is an L-Band sensor with three beams pointing cross-track in a pushbroom fashion producing an approximately $380 \mathrm{~km}$ measurement swath [1]. 
MWR is designed to make eight equally spaced measurements collocated along the AQ swath. The instrument comprises three Dicke radiometer receivers and two reflector antennas; Ka-band looking forward and K-band looking aft. Operating in a pushbroom mode, each antenna produces eight instantaneous field of views (IFOVs) on earth's surface along two conical arcs at earth incidence angles (EIA) of $52^{\circ}$ and $58^{\circ}$.

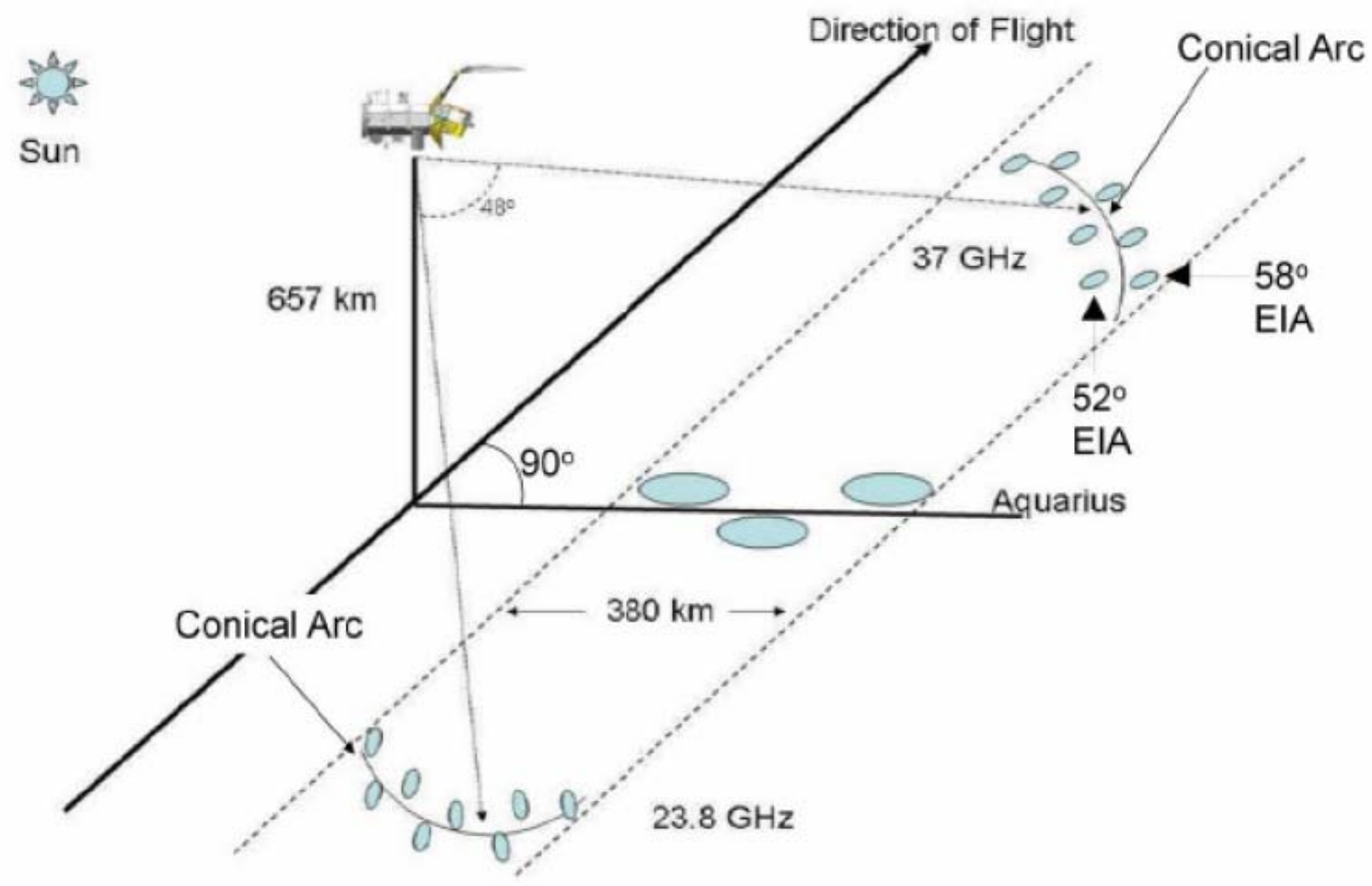

Figure 1-1 Aquarius and MWR measurement geometry 


\subsection{MWR Geolocation Description}

From the standpoint of MWR, geolocation refers to the geographical location of the center of the antenna beams IFOVs on the Earth's surface. Determining this location is a multistep process that is based on two coordinate systems: 1) the satellite's instantaneous location in its orbit relative to an earth-centric coordinate system and 2) the satellite platform geodetic (pointing normal to earth’s surface) coordinate system [4].

First, the satellite must be located in its orbit. The orbit of Aquarius/SAC-D is sun synchronous as determined by Kepler’s Laws [5], which allows dynamic knowledge of the satellite location relative to the center of the Earth. The instantaneous satellite location is given as a three component vector in spherical coordinates $(r, \theta, \varphi)$, where $r$ is the distance from the center of the earth to the satellite, $\theta$ is latitude and $\varphi$ is longitude. This vector defines where the satellite is located at a given instant of time and its location can be determined to a high level of accuracy (typical uncertainties $<1 \mathrm{~m}$ ).

Next, the satellite platform geodetic Cartesian (X, Y, Z) coordinate system is used to locate the IFOVs on the Earth’s surface. On orbit, the satellite continually rotates in pitch (360\% \% orbit) such that it's “-Z axis” is always pointed normal to the surface, and the direction of flight lies along the X-axis. The MWR antenna beam pointing is relative to the satellites Z-axis such that the antenna beams view the surface at a fixed cone angles and varying azimuth angles to the right-hand side of the spacecraft. Using these beam pointing cone and azimuth angles, the beam boresight (center of the IFOV) can be determined on the Earth's surface at a given instant in time, taking into account the instantaneous spacecraft attitude (roll, pitch and yaw). However, the geometry becomes more complicated as the velocity of the spacecraft and the rotation of the 
Earth about its polar axis are taken in to account. This is especially important because the MWR beams look both forward and aft of the satellite direction of flight and the Aquarius footprints.

It is crucial that the MWR surface footprints be properly geolocated. After the Aquarius and MWR sensors were turned on in August 2011, this became a major priority. Many things can cause error in geolocation e.g., satellite orbit position errors, satellite attitude mispointing, improper alignment of the instrument onto the satellite platform, etc. If the entire satellite were misaligned the error would be evident in the Aquarius IFOV geolocation as well as the MWR. However, if the error is instrument based then only that sensor would have geolocation disagreement. These differences can be determined by comparing the MWR forward and aft beam alignment results and comparing MWR to Aquarius mispointing calculations.

\subsection{Thesis Objectives}

The main objective of this thesis is to quantify the mispointing error of the center of MWR IFOV's for all beams on all three channels (23.8 GHz horizontal polarization, and 36.5 GHz vertical and horizontal polarizations). This is accomplished by comparing MWR observed land/water boundaries with surface truth in the form of a high-precision coastline land map. MWR observed boundaries are determined by the point of maximum brightness temperature slope during the transition from the radiometrically cold ocean scene to the radiometrically hot land scene (and vice versa). This method has been used in the past for WindSat and SSMI and has been proven to be quite successful [6] [7].

For this thesis, an algorithm was developed to determine locations of land/water crossings and then compute the differences, compared to the coastline map, on a near-global 
scale. Patterns emerge from comparison of these MWR observed boundaries between separate beams, ascending/descending orbits, land-to-water/water-to-land, latitude, and angle of IFOV/coastline intersection. After extensive analysis, geolocation errors can be estimated for each beam. Further, if all beams produce similar errors, then a generalization can be made for each channel or the entire sensor.

This thesis is organized into five chapters. Following this introduction, Chapter 2 gives a detailed description of: the MWR sensor, the data used for the assessment algorithm, and the errors that can occur in this geolocation analysis. In Chapter 3, the MWR geolocation assessment algorithm is defined. The flaws that are inevitable when using this method and the ways to mitigate these errors are also described in Chapter 3. The fourth chapter presents the MWR geolocation validation results based upon on-orbit MWR measurements. Finally, in Chapter 5, conclusions are presented and suggestions for future work are given. 


\section{CHAPTER 2: MWR DATA}

\subsection{Aquarius Instrument}

The Aquarius L-Band radiometer/scatterometer employs a three meter offset parabolic reflector to project three beams on the Earth's surface perpendicular to the spacecraft's direction of flight. This radiometer system is a passive instrument with a center frequency of $1.4 \mathrm{GHz}$; it has three separate radiometers each with a single feed horn. It is the main instrument for salinity measurements. The active instrument, the Aquarius scatterometer, operates at $1.26 \mathrm{GHz}$ to obtain sea surface wind speed. Ocean surface winds cause an increase in brightness temperature, and if these effects are not properly removed can result in inaccurate brightness temperature measurements. These two instruments utilize the same feed horns generating perfectly coincided footprints spanning $380 \mathrm{~km}$. This pattern produces global coverage every 7 days.

\subsection{Microwave Radiometer}

MWR is another microwave instrument on the AQ satellite. It is comprised of two Dicke switch radiometers utilizing $\mathrm{K}$ and $\mathrm{Ka}$ frequency bands centered at $23.8 \mathrm{GHz}$ (vertically polarized) and $37.5 \mathrm{GHz}$ (vertically and horizontally polarized), respectively. The switch matrix for each band consists of eight feed horns which receive reflected earth surface radiation from an offset parabolic reflector as shown in Figure 2-1. This radiation is then sent through waveguide to a switch matrix for processing [8]. 


\section{Aft pointing Forward pointing}

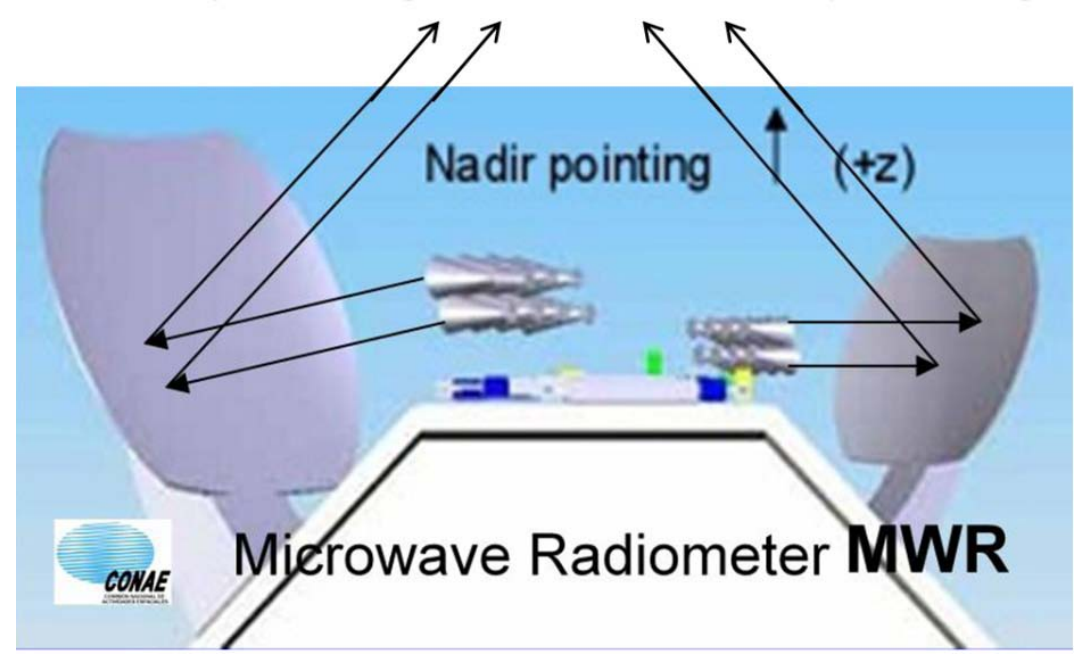

Figure 2-1 Image of MWR feed horns and reflectors

The Ka band reflector is pointed ahead of the spacecraft, and the $\mathrm{K}$ band reflector is directed aft; both take measurements in a pushbroom fashion. Each frequency's eight beams lie along conical arcs on earth’s surface and alternate between an EIA of $52^{\circ}$ and $58^{\circ}$. Because the feed horns are side by side, each beam alternates EIA to prevent signal contamination. The MWR IFOVs are aligned to perfectly overlap Aquarius’380 km swath at an off nadir distance of $272 \mathrm{~km}$ producing a cone angle of $\sim 48$ and azimuth angles ranging from 16-60 .

Each beam is sampled for $240 \mathrm{~ms}$. Since there are eight beams, this leads to an integration time of 1.92 seconds. During a beam’s 240 ms sample the radiometer repeats eight cycles. Like a typical Dicke switch radiometer, one cycle consists of MWR observing the antenna temperature, then the antenna plus random Gaussian white noise, and finally a reference load. This sampling process is demonstrated in Fig. 2-2. 


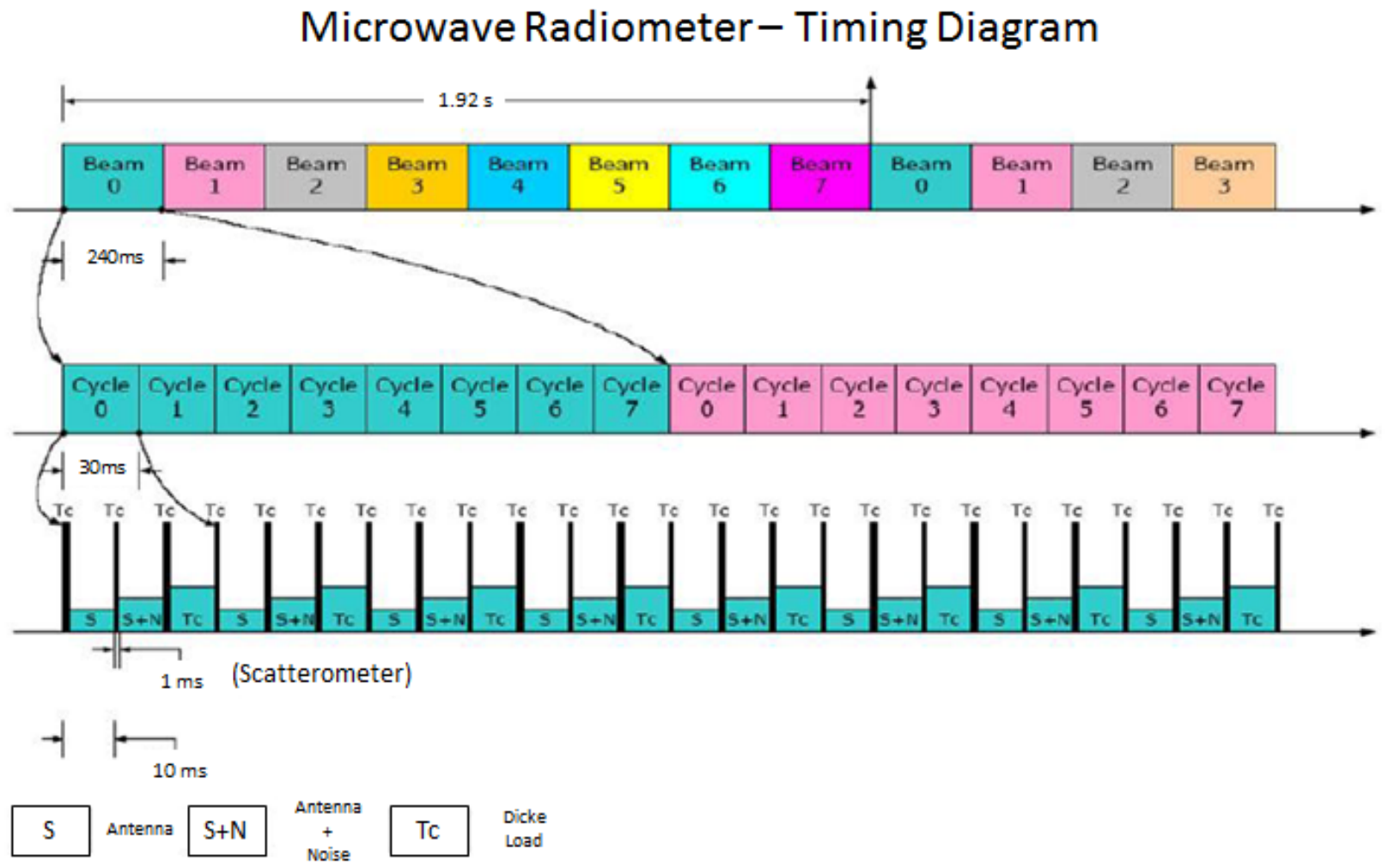

Figure 2-2 MWR Beam Sampling

Aquarius has a ground velocity of $6.82 \mathrm{~km} / \mathrm{sec}$. After applying this velocity to the previously mentioned integration time of 1.92 seconds, the integration distance between sampled IFOVs is approximately $13.1 \mathrm{~km}$. Because the IFOVs are approximately 30x60 km, the beams will overlap in the along track direction.

\subsection{Algorithm Overview}

When an MWR beam crosses over a land/water boundary there is a large change in brightness temperature from a radiometrically hot land scene to a radiometrically cold ocean scene. This transition can be defined by its slope, TB/MWR sample, For this algorithm, it is first proved, then assumed that the boundary (where the antenna beam pattern views half land values 
and half ocean values) is observed at the MWR point of maximum slope. The error of MWR geolocation can be measured by comparing the location of this maximum slope, and a $1 \mathrm{~km}$ resolution land mask, provided by Remote Sensing Systems (REMSS) and taken as truth.

\subsection{Data used for algorithm}

The three input parameters needed for this geolocation assessment are: MWR track latitude, longitude, and brightness temperature (Tb). These three parameters are plotted on earth maps in figures 2-3 and 4. The MWR Tb is used to calculate Tb slope for each beam and is used to define the land/water boundary; however, these spatial location data are needed for determining distance from the coast and must be carried along for later calculations.

All orbits for the week of November 14-20, 2011 are used in this analysis because this represents the satellite ground-track repeat period. Using multiple weeks of data will produce the same amount of land/water boundaries in the same locations. MWR measurements below $40^{\circ}$ South latitude and above $60^{\circ}$ North latitude were not used to avoid transient and unknown ice boundaries.

A statistical analysis of geolocation errors is presented in Chapter 4, where these data are described by five output criteria: beam number (eight beams at each frequency), ascending or descending orbit, and land to water or water to land crossings, latitude of crossing and angle of MWR intersection with the coastline. Analysis is performed by beam because the antenna feed alignments are independent. Separation of the data by ascending and descending pass, angle of intersection and latitude of crossing is used to detect static satellite attitude roll, pitch and yaw offsets. The land to water and water to land criteria is used to validate that an error correction for boundary type does not need to be implemented. 


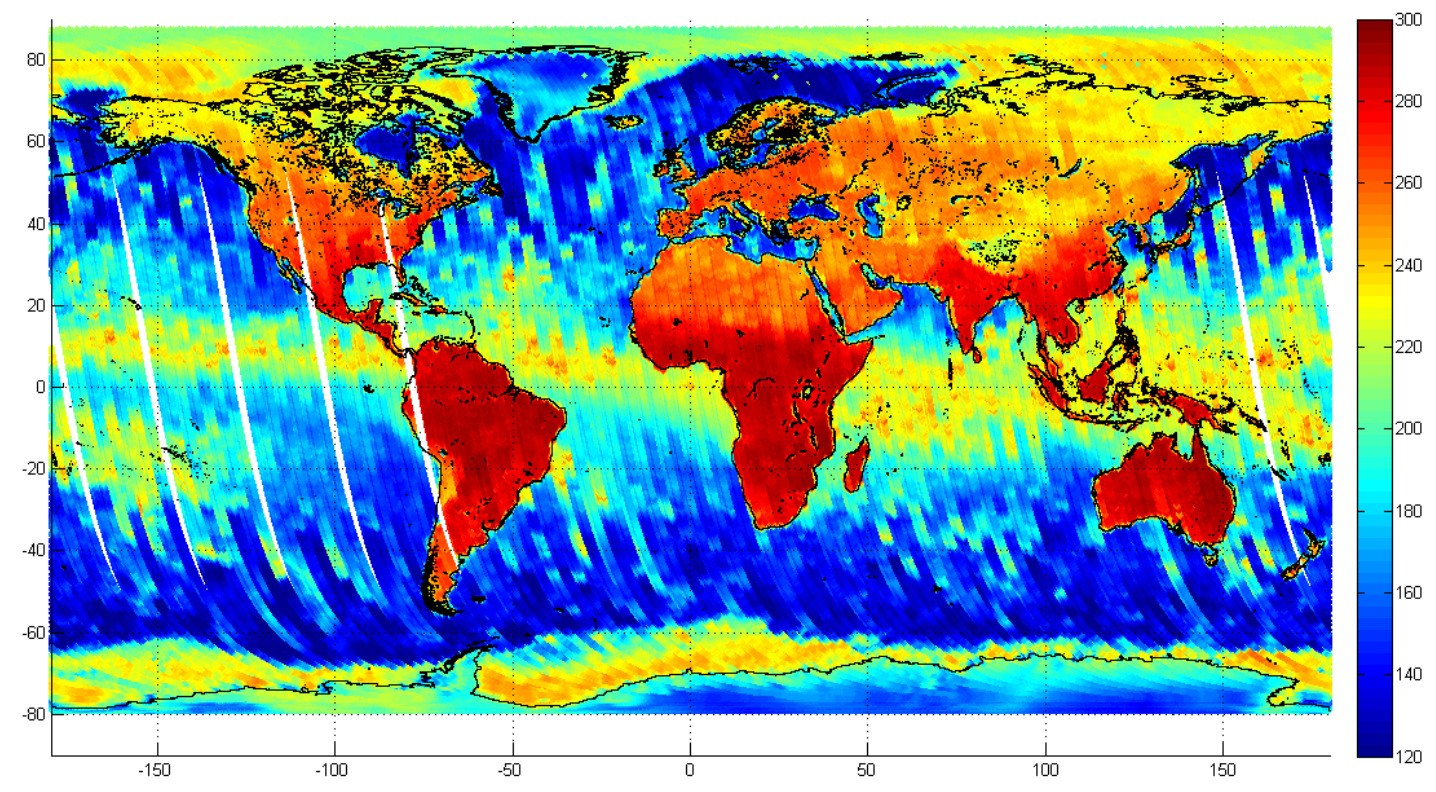

Figure 2-3 All ascending passes used for geolocation assessment (Nov. 14-20, 2011).

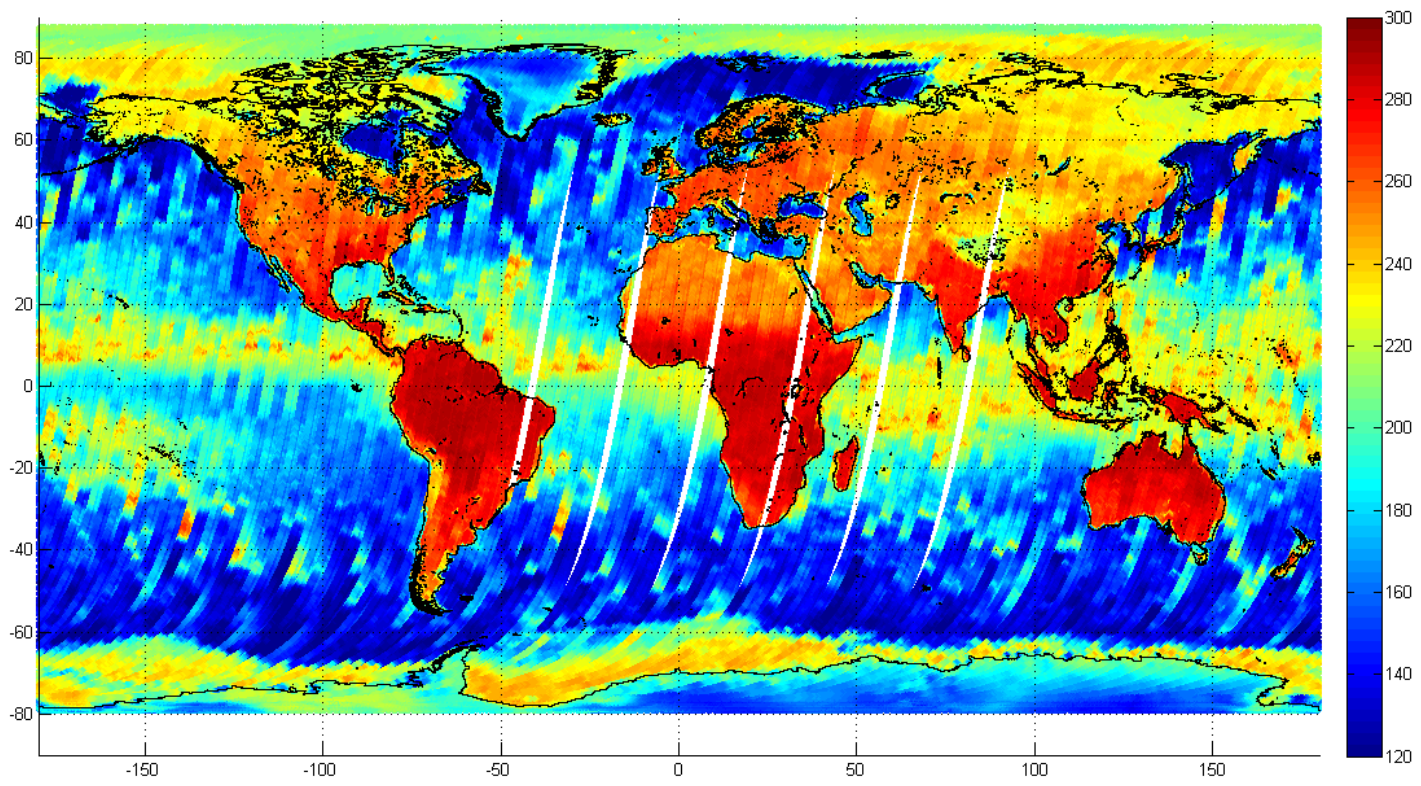

Figure 2-4 All Descending passes used for geolocation assessment (Nov. 14-20, 2011). 


\subsection{Errors that can be calculated}

As mentioned previously, the end goal is to isolate geolocation errors. Many types of error exist and a few are outlined here. Satellite attitude and sensor mounting errors can be characterized by the same type of error namely roll, pitch and yaw. The effects of satellite and sensor error emerge when forward and aft beam geolocation errors are compared.

\subsubsection{Antenna Pattern Error}

An antenna pattern describes the power intensity at various elevation and azimuth angles off boresight directed to the feed horn from the antenna. Each horn has an individual antenna pattern. Asymmetry about the boresight is typical of most horns, and can contribute to a slight geolocation error. The geolocation error attributed to the antenna pattern can be characterized in terms of brightness temperature mislocation on the Earth's surface; however, the maximum $\mathrm{Tb}$ slope location at a boundary is less affected by this asymmetry so the antenna pattern error is not specifically calculated in this thesis. 


\subsubsection{Roll Error}

Roll, pitch, and yaw errors present distinct characteristics as a beam passes over a land/water boundary. Figure 2-5 is an example of roll error. By comparing ascending and descending orbit passes this error can be evaluated. When the satellite is positively rolled the land water boundaries on near parallel crosses will be shifted to the left for both the ascending and descending pass. The west and east coasts of continents are helpful for quantifying this error.

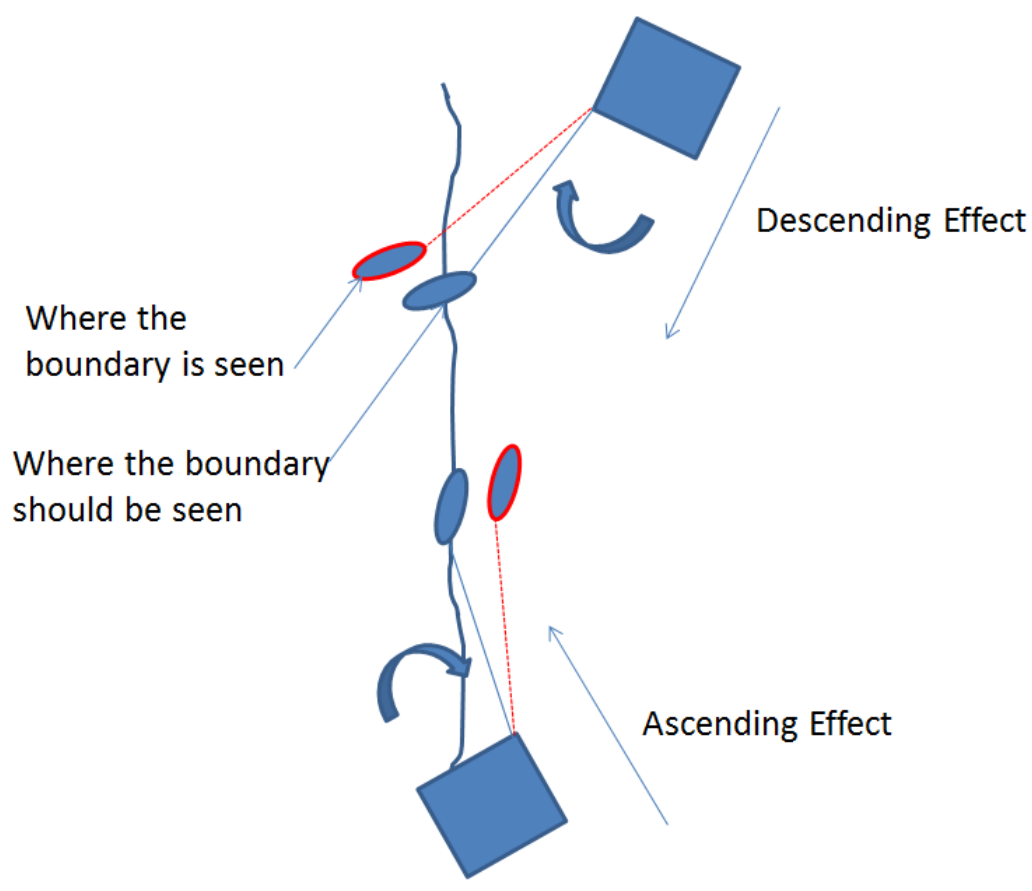

Negative Roll Error

Figure 2-5 Negative roll causes the beam to be displaced to the right on Earth's surface 


\subsubsection{Pitch Error}

There are multiple ways that pitch error can be quantified: displacement of ascending and descending passes on northern and southern boundaries of the continents, comparing the boundary location of forward and aft beams, and isolating near perpendicular boundary intersection angles. The ascending and descending comparison is demonstrated in Fig. 2-6. All boundaries would consistently see the boundary before or after its true location. However, on the continental boundaries this would be evident by the separation of a similarly located ascending and descending pass. Pitch can also be assessed by the magnitude of error distance between forward and aft beams. If the spacecraft was positively pitched, the forward beams would have a larger error distance that the aft beams.

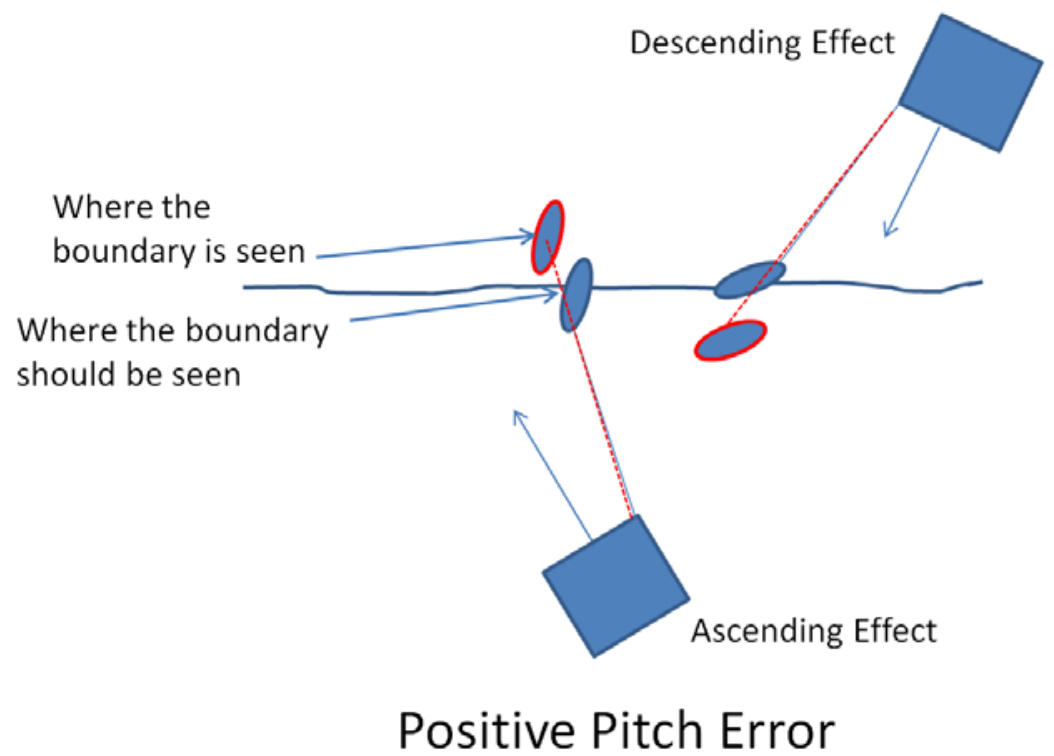

Figure 2-6 Positive pitch means the front is angled higher than the rear of the spacecraft 


\subsubsection{Yaw Error}

Yaw is classified as the rotation of the satellite around the vertical axis. An advantage to the MWR is that it has multiple beams, at varying incidence and azimuth angles on Earth's surface at any given time. Yaw error can be assessed by comparing the relative location of these footprints over time. Due to the movement of the spacecraft and the rotation of the Earth, the location of these beam footprints would naturally drift. However, one important requirement for this mission is that the MWR footprints perfectly overlap the Aquarius swath to provide ancillary data to Aquarius.

This shift is sinusoidal. The yaw error that will occur is greatest at the equator, and drops off at the poles. Yaw steering has been implemented to counteract this drift [9]. By using the forward and aft beams of MWR, yaw error can be determined and the effectiveness of yaw steering can be validated. The methodology for this analysis is represented in Fig. 2-7.

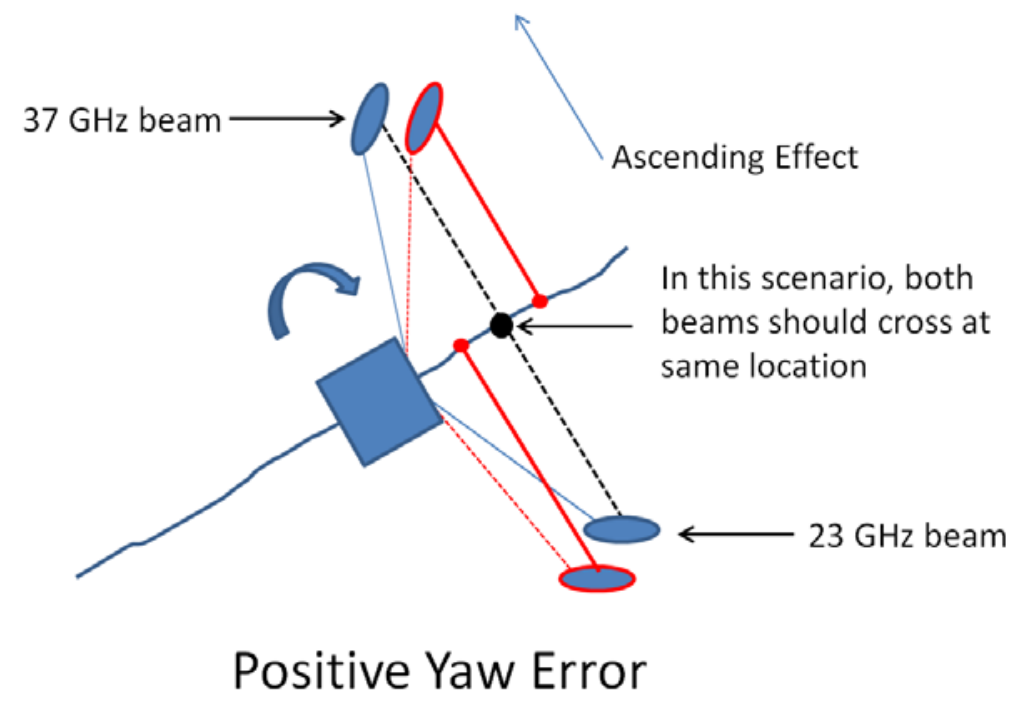

Figure 2-7 Yaw error causes the forward and aft beams to cross the boundary at different locations 


\section{CHAPTER 3: GEOLOCATION ASSESSMENT ALGORITHM}

\subsection{Method Overview}

The MWR data used for this algorithm are a time series of vectors of IFOV center latitude and longitude, and Tb. Major changes in Tb occur when the MWR antenna views ocean and land as shown in Fig. 3-1. Although the dynamic range of these changes depends upon the radiometer frequency, earth incidence angle (EIA) and polarization, the land/water boundaries typically produce $>100 \mathrm{~K}$ brightness temperature contrast, and the largest rate of change in $\mathrm{Tb}$

per sample (Tb slope) is an efficient way of characterizing the location of a land/water boundary.

\subsection{Experimental Observation of Land/Water Boundary}

Previous investigators [6][7] have used the rapid change in Tb at the location of the land/water boundaries to determine the antenna pointing accuracy, and their approach showed that the measured $\mathrm{Tb}$ slope was a maximum at this boundary. We intend to apply a similar approach to the MWR antenna IFOV geolocation validation; however before we do, it is prudent to demonstrate that the maximum slope does indeed occur at the land/water boundary. We use simulation to demonstrate this principle as described below.

The first step of the simulation is to model the ideal Tb transition from water-to-land using the observed 37-H GHz channel ocean and land brightness temperatures at approximately $3 \mathrm{~km}$ steps on the surface. As shown in Fig. 3-2, the resulting theoretical brightness temperature at the water-to-land boundary is a 'step-function', where the transition occurs at an x-value equal to 300. The first 200 points are assigned an ocean value of $130 \mathrm{~K}$, the center point is assigned a 
value of $203.5 \mathrm{~K}$ (mean of land and ocean brightness temperatures), and the last 200 correspond to land and are set to $277 \mathrm{~K}$. 


\section{Tb $23 \mathrm{GHz} \mathrm{Hpol}$}

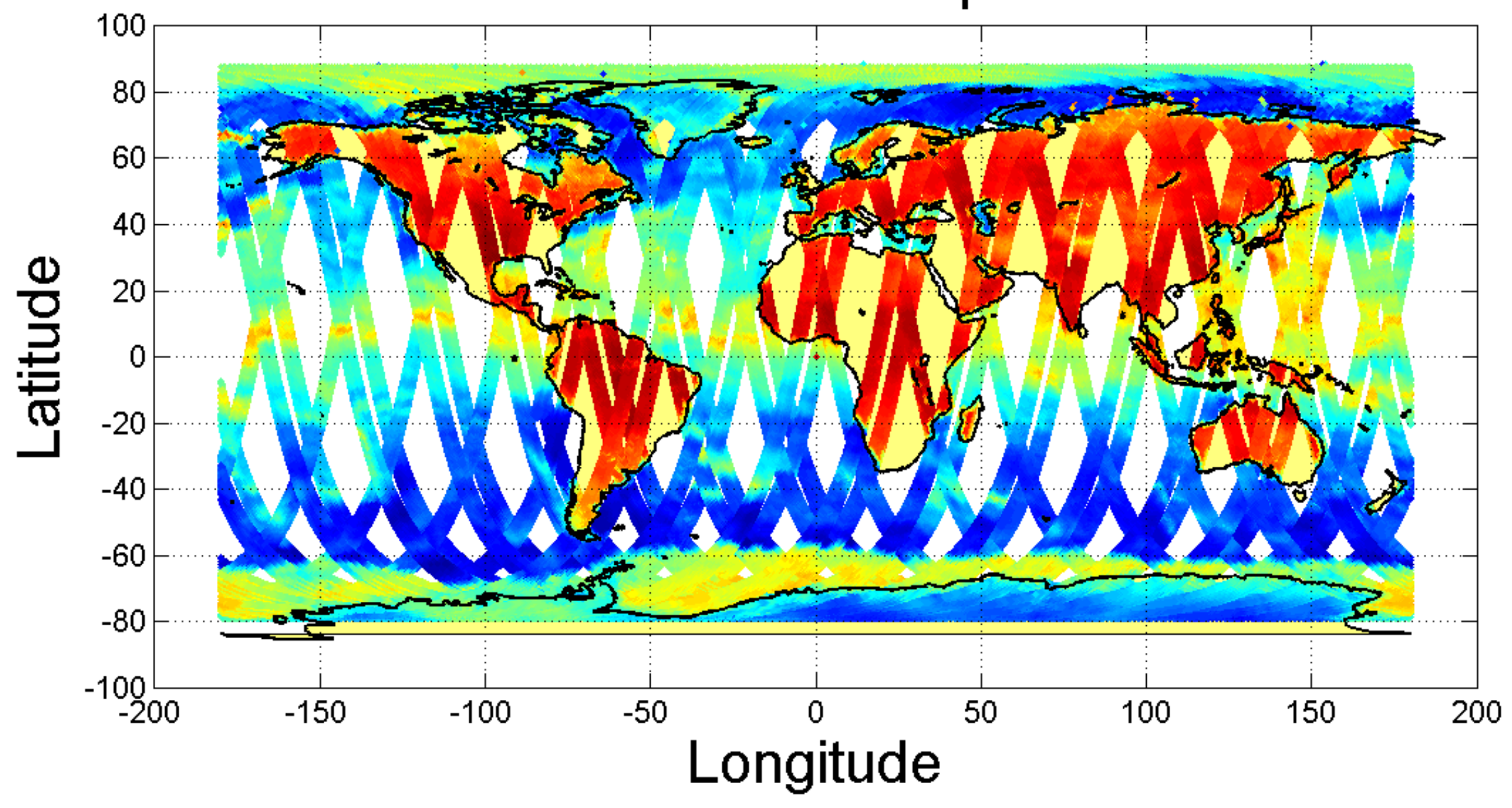

Figure 3-1 MWR track, all eight beams, approximately 20 orbits. The ocean is radiometrically 'cold', and the land is 'hot'. 

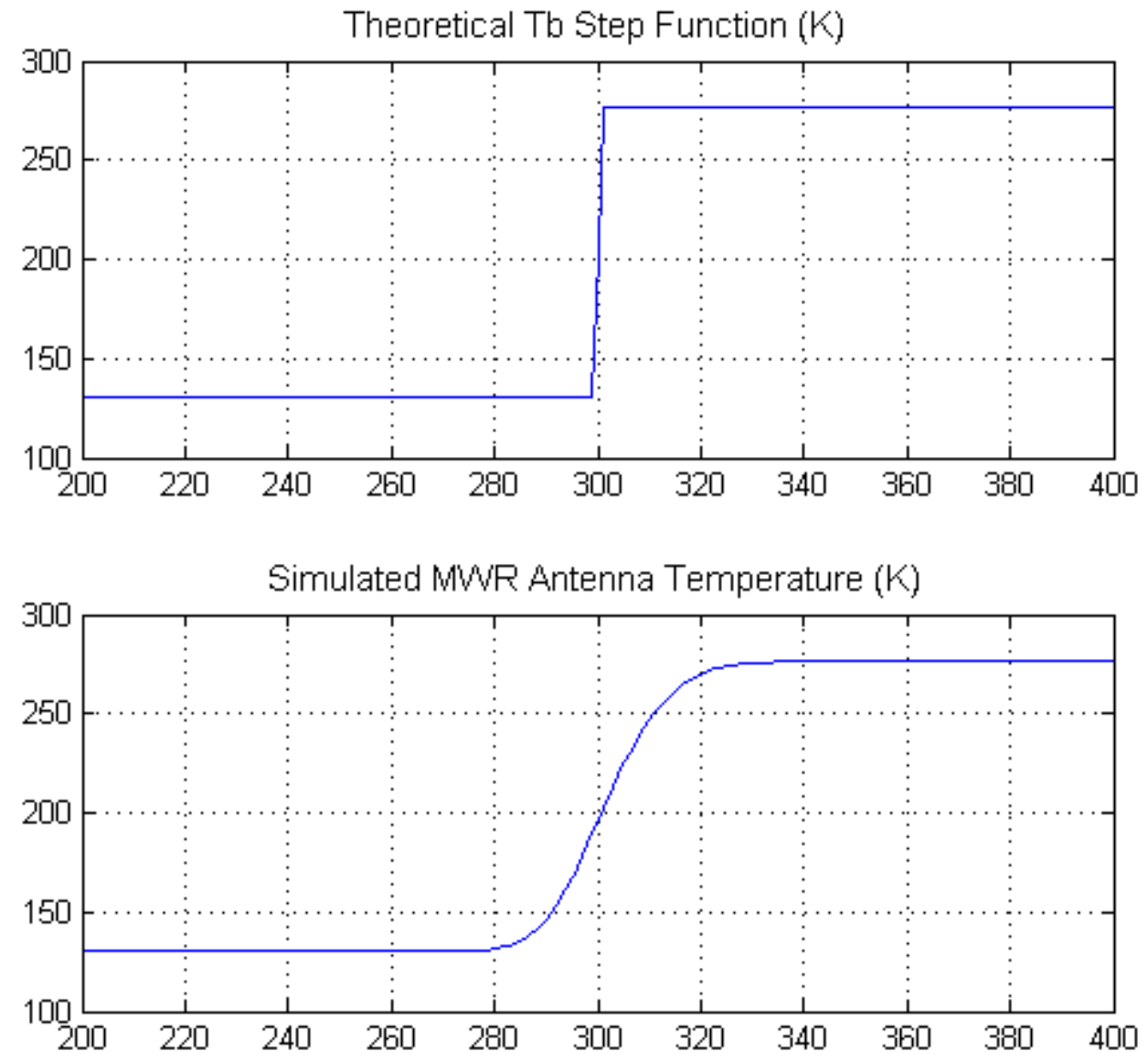

Figure 3-2 Simulated brightness temperatures: upper panel is the theoretical apparent Tb for an ocean/land transition and lower panel is the simulated MWR antenna temperature. 
The second step involves the simulation of the MWR brightness temperature measurement as the IFOV crosses the ocean/land boundary. This process, illustrated in Fig. 3-3 and described in Ulaby, Moore and Fung, involves the weighted summation of the scene apparent brightness temperature by the antenna pattern (known as the antenna pattern convolution integral), which yields the antenna (brightness) temperature given by:

$$
T_{A}=\frac{\iint_{4 \pi} T_{a p}(\theta, \varphi) * F_{n}(\theta, \varphi) * \sin \theta d \theta d \varphi}{\iint_{4 \pi} F_{n}(\theta, \varphi) * \sin \theta d \theta d \varphi}
$$

Where $\theta$ is the antenna pattern elevation angle, and $\varphi$ is the pattern azimuth, $T_{a p}$, is apparent scene brightness temperature, which in our scenario, is the brightness temperature step function, $F_{n}$ is the linear antenna pattern, and $T_{A}$ is the output brightness temperature of the antenna.

For our one-dimensional convolution, we are using only the elevation plane antenna patterns $\left(F_{n}(\theta)\right)$ which eliminates other spherical coordinate, $\sin (\theta) \mathrm{d} \varphi$; therefore, the equation can be reduced to this simplified version.

$$
T_{A}=\frac{\sum_{\theta=-27}^{27} T_{a p}(\theta) * F_{n}(\theta)}{\sum_{\theta=-27}^{27} F_{n}(\theta)}
$$

For Eq. 3-2, we evaluate the integral numerically using the maximum antenna pattern resolution, which is $\Delta \theta=0.1^{\circ}(\sim 3 \mathrm{~km}$ steps $)$ and $\theta$ ranging from -27 to $27^{\circ}$. Thus, the convolution integral yields the simulated antenna temperature time series with a sampling of approximately $3 \mathrm{~km}$ on the surface that is shown in the lower panel of Fig. 3-2. Later we will resample this high-resolution theoretical antenna temperature time series using the MWR 
sampling rate that occurs every $13 \mathrm{~km}$ on the surface (corresponds to $\sim 0.4^{\circ}$ in off-boresight angle). 


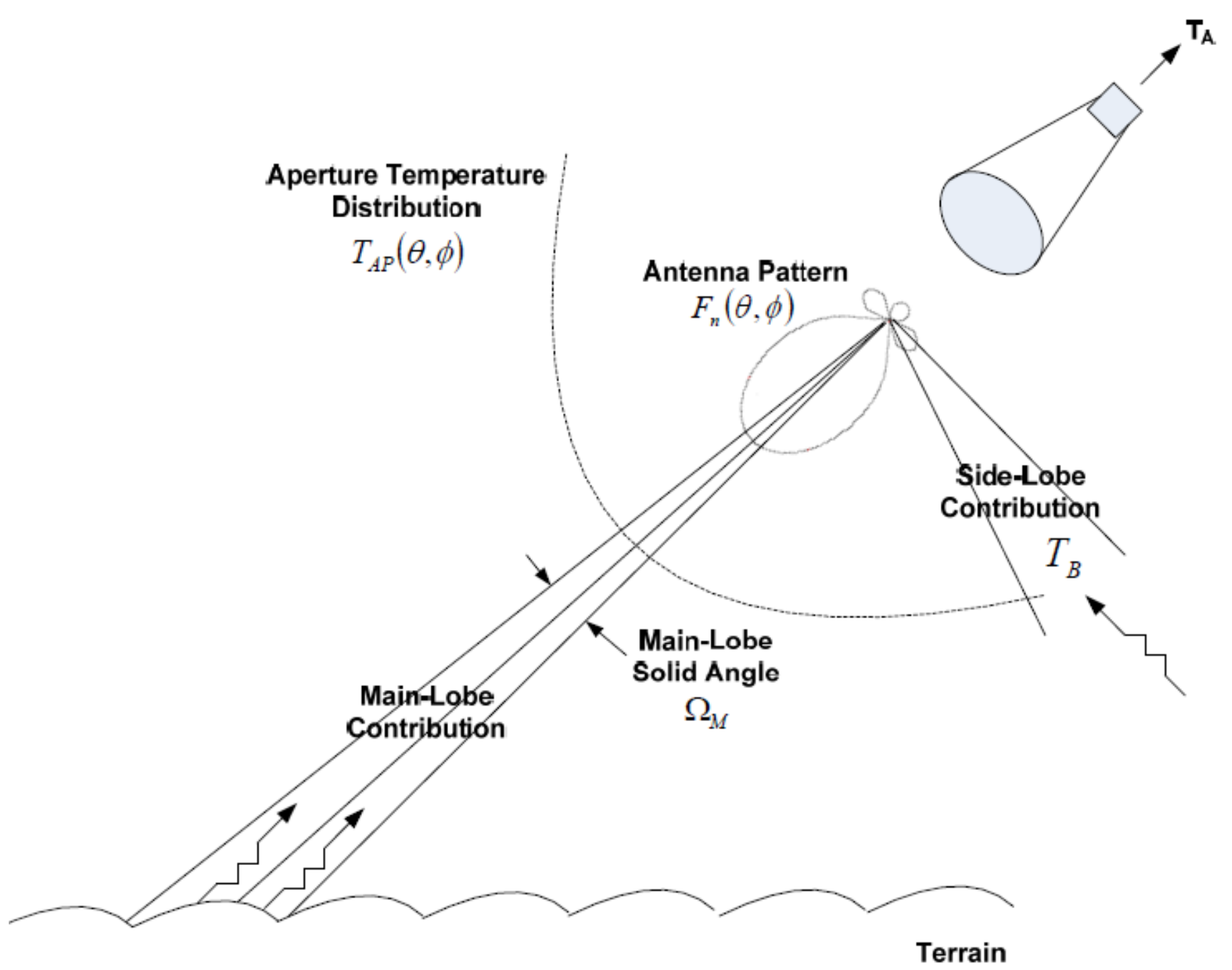

Figure 3-3 Antenna temperature definition 
As mentioned previously, the antenna pattern varies slightly for all 24 beams; and as an example, the beam-3, 37-H channel antenna pattern from the prelaunch anechoic chamber test was chosen [11]. This antenna beam relative directivity pattern, given in Fig. 3-4 (dB units) and Fig. 3-5 (linear units), is the elevation-plane cut for off-boresight angles of $\pm 27^{\circ}$ in $0.1^{\circ}$ steps. A slight asymmetry is apparent in this measured antenna pattern, when compared to a theoretical, symmetrical Gaussian of the same half-power beamwidth (Fig. 3-5).

The convolved antenna temperature for the Gaussian beam (performed in $0.1^{\circ}$ steps) is shown in Fig. 3-6. When the boresight of this Gaussian beam points directly at the boundary (xvalue $=300$ ), the two halves of the elevation antenna pattern observe land or water respectively ( $50 \%$ beam-fill fraction). As expected, the boundary antenna temperature is exactly $203.5 \mathrm{~K}$, and the location of the maximum slope occurs precisely at step 300 (see Fig. 3-6).

However, when the actual MWR antenna pattern is used for the convolution, then the corresponding location of $203.5 \mathrm{~K}$ moves to the right to an $\mathrm{x}$-value of 301.25 . Thus, the location of the boundary occurs later than expected because the antenna pattern favors the lagging side, which lowers the antenna temperature. The distance on the Earth surface corresponding to the shift in the location of the maximum slope of $0.125^{\circ}$ is $\sim 3.75 \mathrm{~km}$.

The third step of the simulation involves determining the geolocation effort based upon the location of the maximum $\mathrm{Tb}$ slope for the antenna temperature time series. Since we performed this simulation, the "true" location of the boundary is known; thus the difference between the MWR observed (convolved) and true boundary represents the geolocation error. Both the Gaussian and the MWR beam-3 pattern were convolved with the Tb step function, which resulted in slight differences in the max slope locations. For the Gaussian, the observed 
max slope location matched the true boundary location (zero error). On the other hand, for the MWR beam-3 pattern, because the antenna beam sidelobes are asymmetric, this causes an apparent "beam-pattern" geolocation error, which is the shift in the location of the maximum Tb slope relative to the "true" land/water boundary. We believe that this bias is stationary and by performing this simulation for all 24 beam patterns, we can characterize this pattern convolution error, which will be applied to the MWR geolocation analysis as a bias associated with each horn antenna pattern.

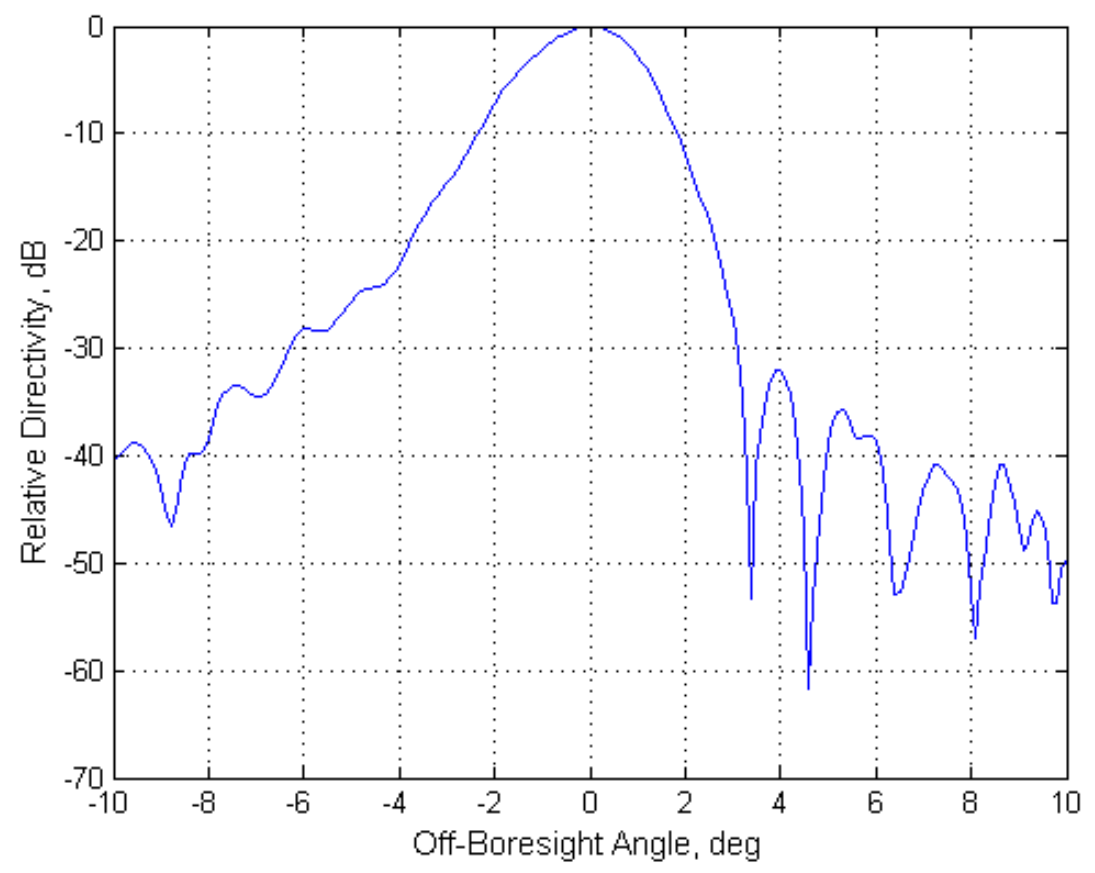

Figure 3-4 MWR 37-H GHz, beam-3 relative antenna pattern 


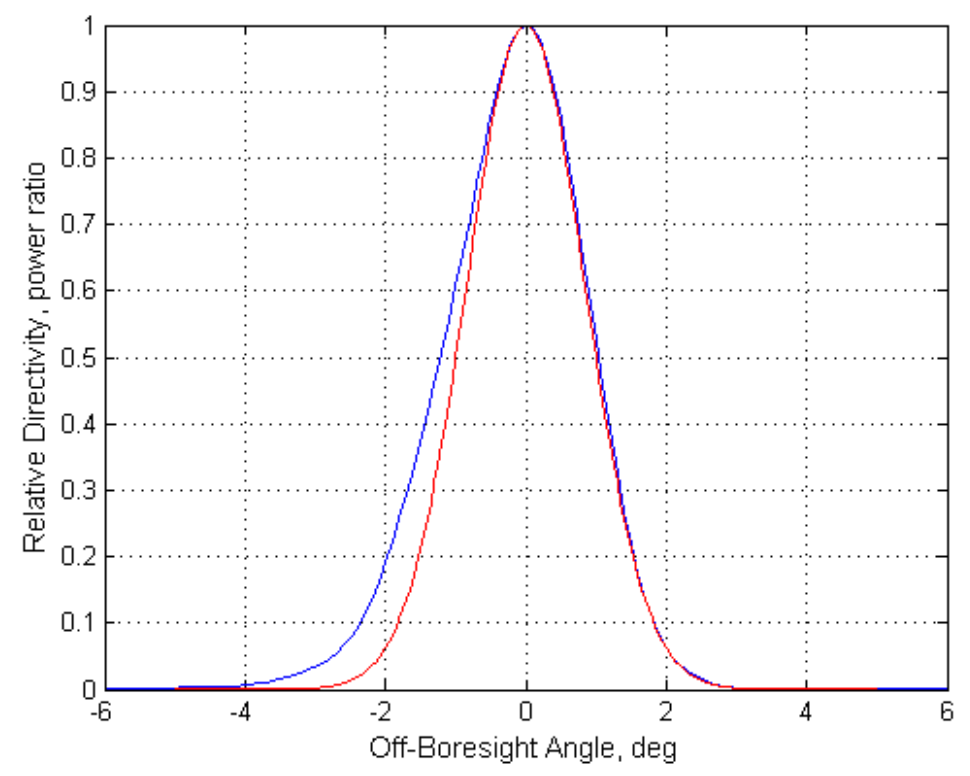

Figure 3-5 MWR 37-H GHz, beam-3, relative directivity pattern (blue), Gaussian pattern (red)

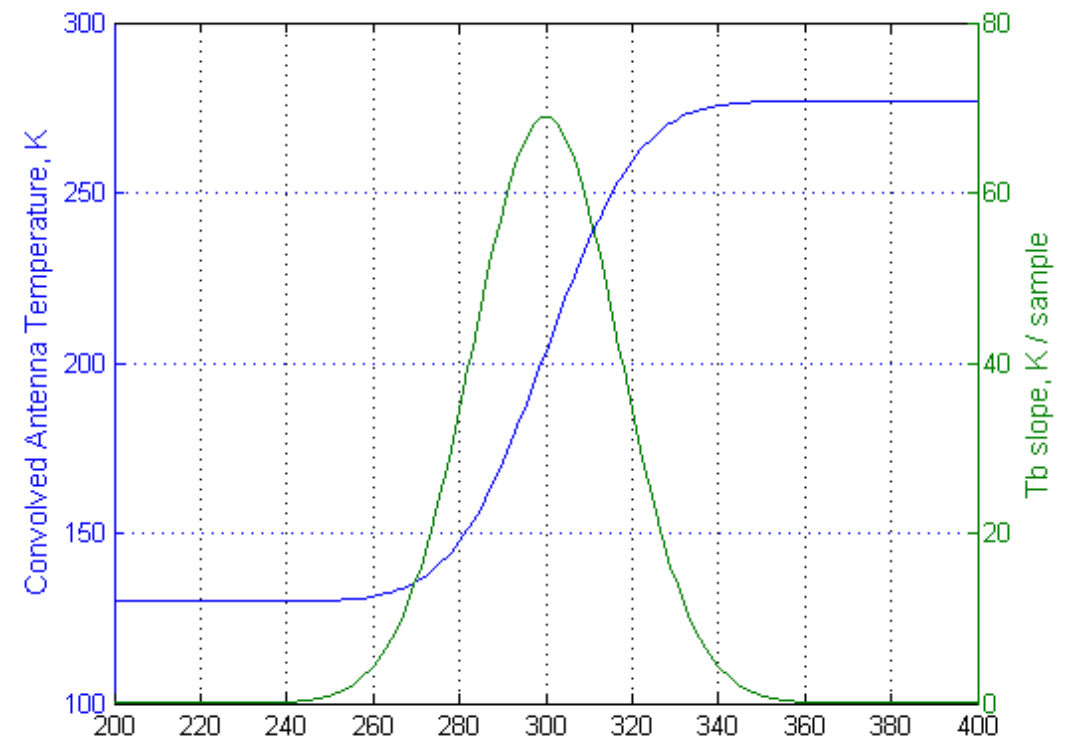

Figure 3-6 Simulated MWR antenna temperature (blue) from Gaussian antenna pattern convolution with step-function surface Tb for an ocean/land crossing. Also shown is the corresponding slope of the convolved brightness temperature (green). 


\subsection{Algorithm Definition}

Figure 3-7 shows a top-level block diagram of the geolocation assessment procedure (algorithm), which is implemented in Matlab. Inputs from the MWR L1B data product comprise; beam\#, antenna boresight latitude, longitude and Tb. The first task is to identify all land/water crossings that occur within 7-day repeating ground-track period (103 orbits). Over this period there are approximately 318,000 MWR observations (pixels)/beam, most of which do not involve coastal crossings; so we use the first three blocks to implement an equivalent band-pass filter to keep only the desired measurements (i.e., near coastal crossings).

First, we impose latitude restrictions to remove the polar regions, where unwanted dynamic boundaries of sea ice exist. Sea ice varies with season, and it would be very difficult to find a map which accurately portrays the ice extent at a given time. Also, this task is unnecessary for this analysis because there are many other land/water boundaries that can be used to characterize the IFOV geolocation error.

Next, we calculate the slope of the MWR brightness temperature separately by beam using a recursive difference between the two surrounding pixels for each MWR pixel. Empirical observations of land/water crossings, reveal that typical $23.8 \mathrm{GHz}$ Tb slopes are $>7 \mathrm{~K} / \mathrm{MWR}$ sample, 36.5-V detects boundaries with slopes > 3.5 K/sample and 36.5-H requires > 8.5 $\mathrm{K} /$ sample. Therefore, we use these values as a threshold to select only the L1B data that are associated with probable land water crossings. Using these thresholds is ideal because the majority of coastal crossings are captured while false boundaries are omitted. 
All MWR Tb’s for each of 103 orbits are passed through this "slope filter" to produce the time series of Tb slopes that occur at land/water crossings. This produces approximately 400 data sets that contain $\sim 5$ points with $13 \mathrm{~km}$ along-track spacings, which reduces the data volume to 2,000 pixels/beam/7-days. Within each data set, the maximum slope value of these threshold points is approximately the location of the MWR sensor observed boundary. But because of the MWR spatial sampling of $13 \mathrm{~km}$, we improve the estimate of the land/water boundary by applying a parabolic fit to the slope curve and finding the location of the parabola maxima.

Once the MWR boundary is determined, two linear equations are developed by regression to represent the MWR IFOV trajectory and the intersected coastline. These linear equations are solved simultaneously to yield the intersection that is the "true land/water crossing” location. Finally, we calculate the distance between the MWR sensor observed boundary (max Tb slope location) and the true coastline, which represents the geolocation error. Output of the geolocation assessment algorithm is error distance for each coastal crossing and other related parameters (e.g., beam number, ascending/descending orbit segment, land-to-water or water-to-land, etc.).

After running the above algorithm, a data set of 6,400 (800 crossings for eight beams) coastal crossing errors were produced and analysis was conducted on each beam for ascending and descending orbit segments. It should be noted that orientation of MWR ground track with respect to the coastline is relatively unimportant; however, the highest slope will occur on the coastline whenever the track of the IFOV center is perpendicular to the coast. Because the orientation of the major axis of the IFOV occurs at an angle of $16^{\circ}-60^{\circ}$ relative to the groundtrack direction, there may be subtle differences in the geolocation error with azimuth angle 
relative to the coastline that will be investigated. The following discussion provides a more detailed description of the algorithm.

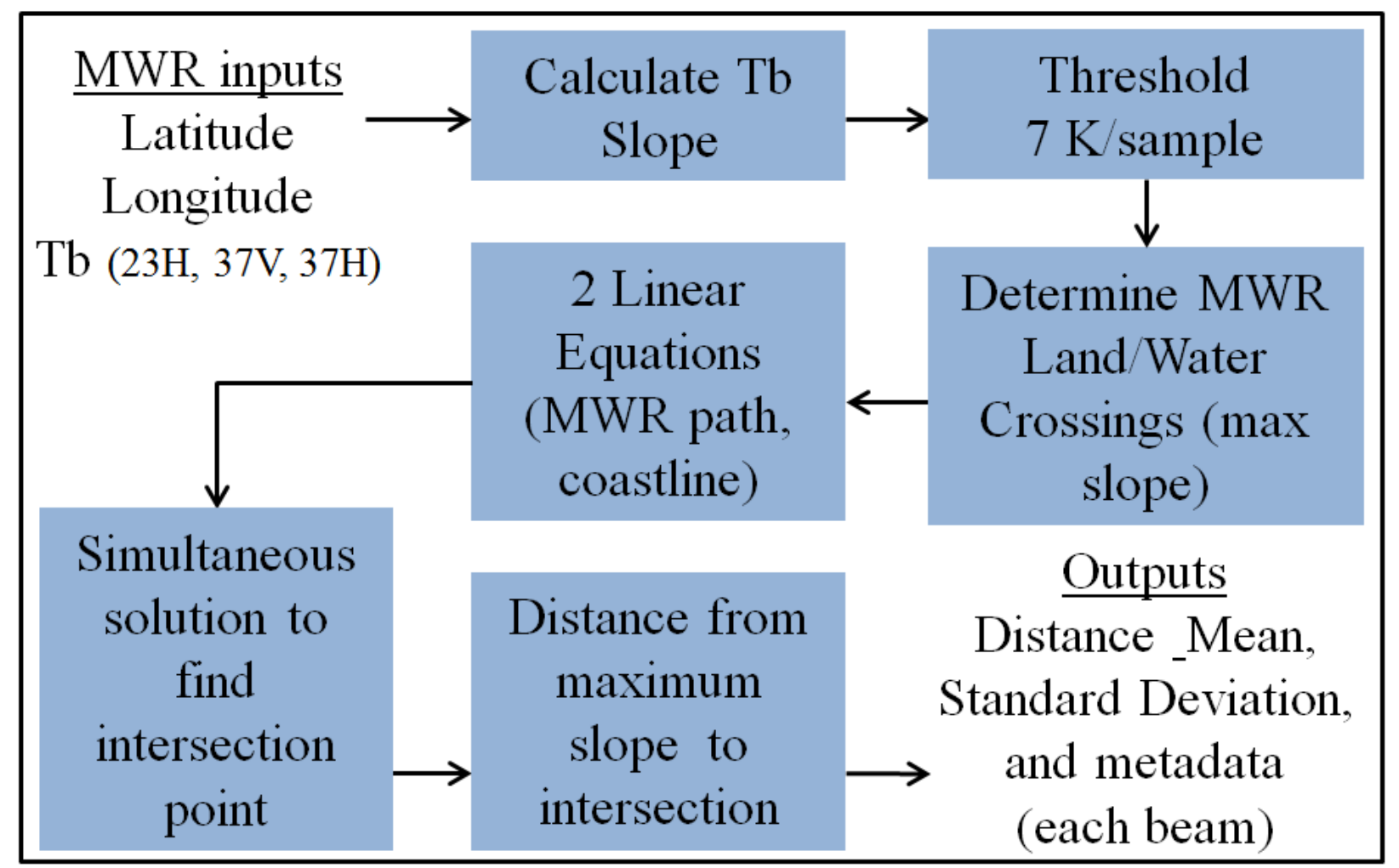

Figure 3-7 Geolocation assessment algorithm block diagram 


\subsubsection{Tb Slope Filter (First three Blocks)}

The first step is to calculate brightness temperature $(\mathrm{Tb})$ slope time series near the land/water transitions. As mentioned previously, the brightness temperature value is assigned to the center location of the IFOV, but the measurement is the convolution of the entire footprint with the Earth's surface. Figure 3-8 is a representation of the size of MWR IFOVs for an ascending pass of 37V channel at a water to land boundary on the west coast of Africa. The brightness temperature for each IFOV across this boundary is given in Fig. 3-9. Using these brightness temperatures, a Tb slope is calculated for each IFOV. For the " $\mathrm{t}^{\text {th" }}$ pixel, this is done with a rise over run equation using the two surrounding MWR Tb observations.

$$
\text { Slope }_{i}=\Delta T b / \text { sample }=\left(T b_{i+1}-T b_{i-1}\right) / 2
$$

As previously discussed, the point of maximum slope is selected as the MWR observed land/water crossing location; however, for practical considerations, we use an empirically determined $\mathrm{Tb}$ slope threshold to remove false-alarms caused by natural Tb variability in the earth scene. Therefore, it is optimum to use only points with slopes larger than an absolute value of $7 \mathrm{~K} /$ sample for $23-\mathrm{H}, 3.5 \mathrm{~K} /$ sample for $37-\mathrm{V}$, and $8.5 \mathrm{~K} /$ sample for $37-\mathrm{H}$ because it minimizes the bogus land/water boundaries while maintaining a large number of samples. Figure 3-10 is an example of the slope threshold test. There are seven points along this MWR coastal crossing that meet or exceed the threshold. Those points are extracted and continue through the geolocation assessment algorithm. 


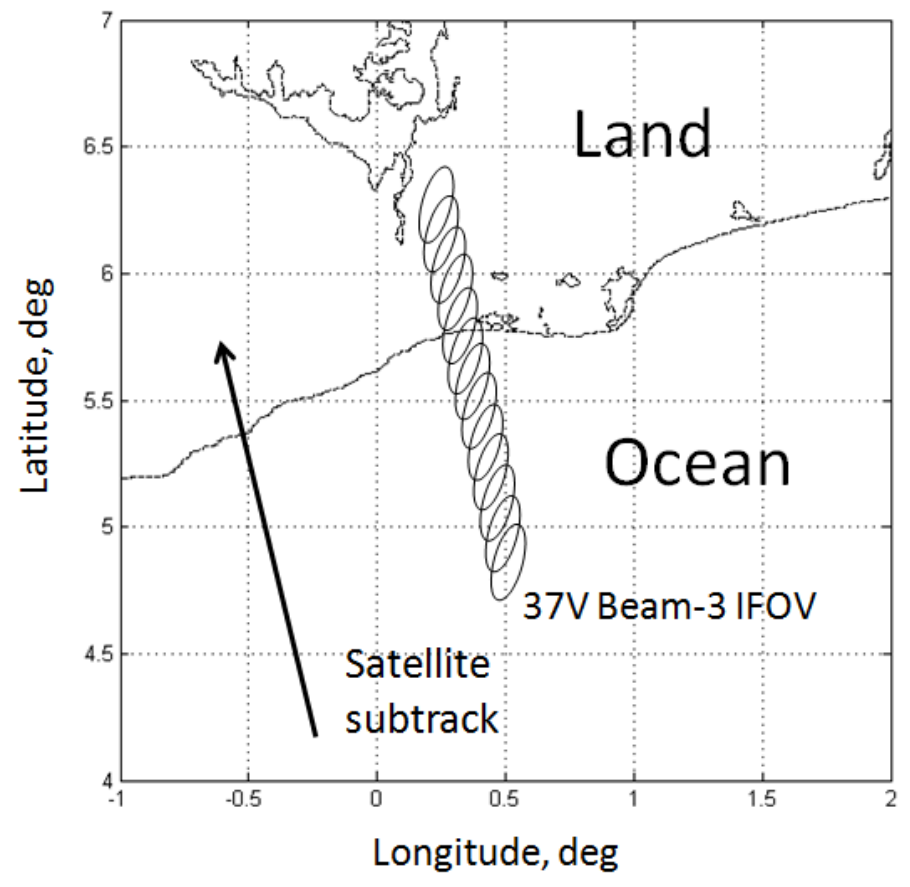

Figure 3-8 Beam 3, 37V IFOVs across a coastal boundary

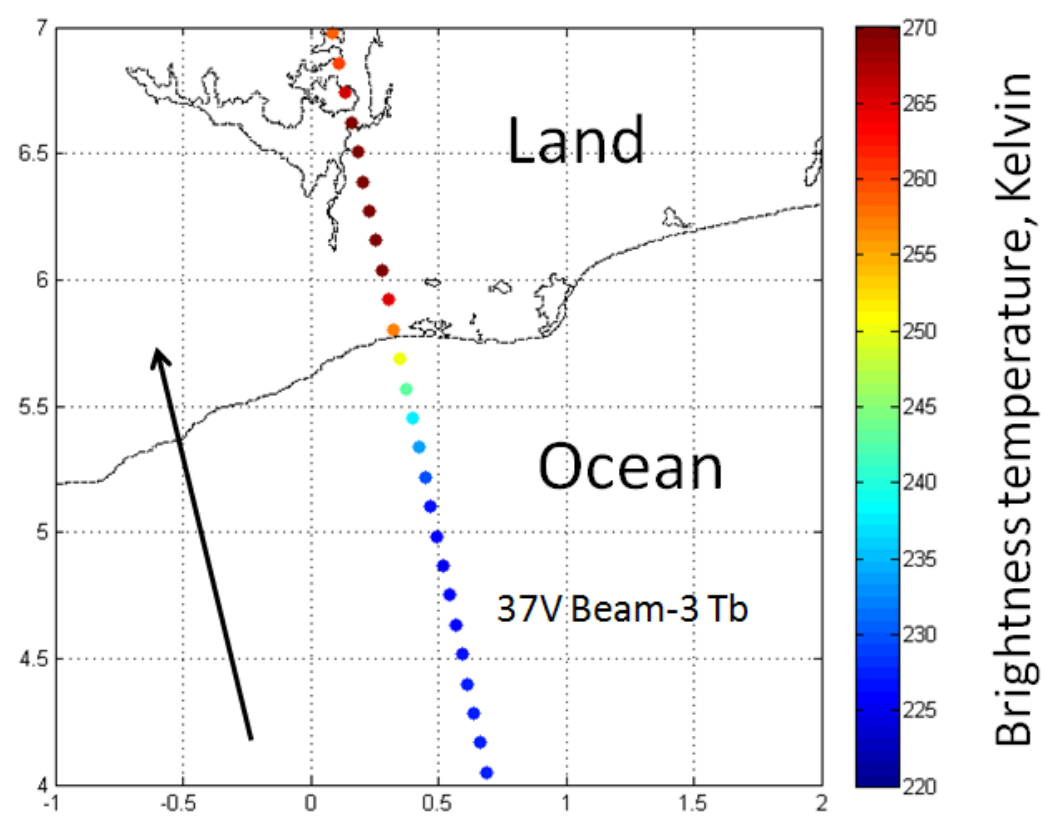

Figure 3-9 Brightness temperature values for coastal boundary 


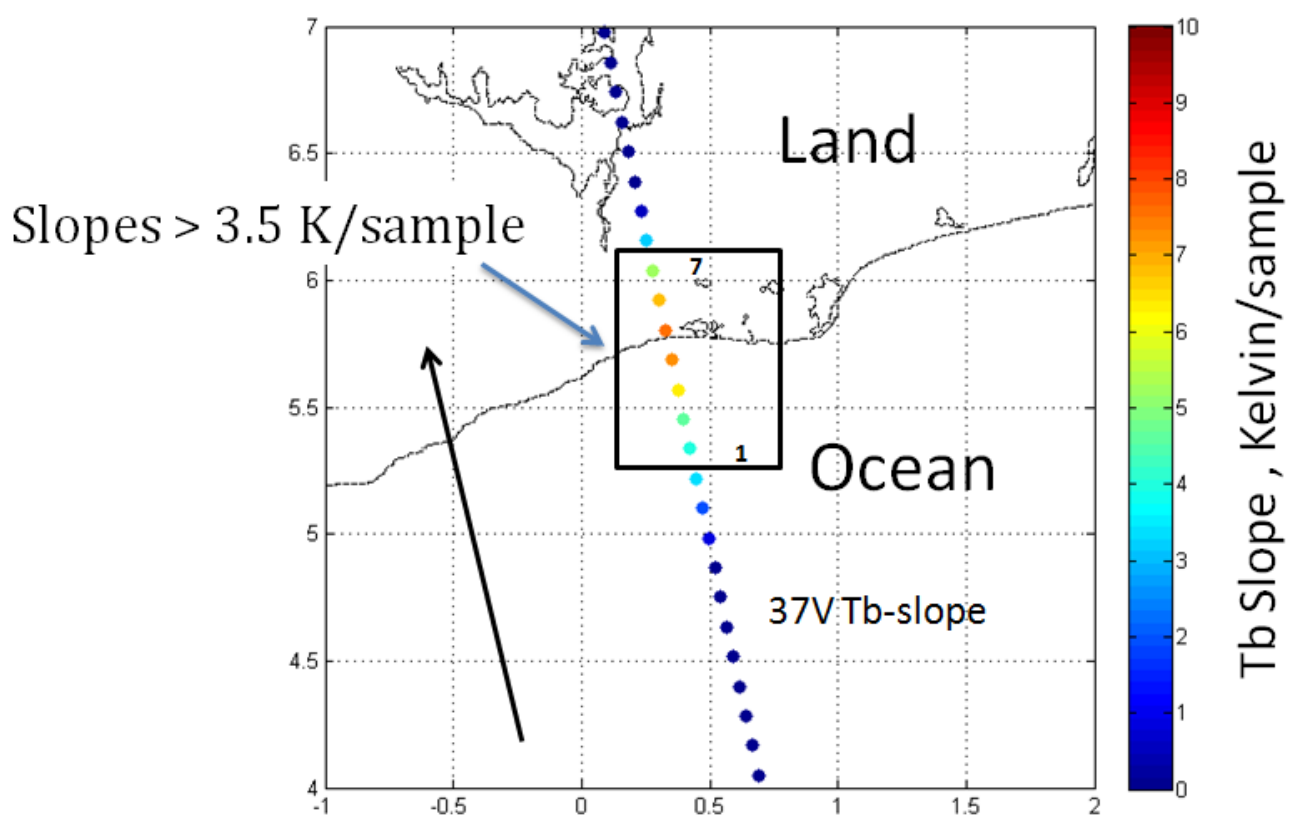

Figure 3-10 Slope threshold applied to a 37V coastal crossing

A second order regression is applied to the points passing the slope threshold test, the latitude/longitude location of each set's maxima is determined to be the crossing location and necessary parameters are extracted. This point of maximum slope at each crossing is considered the sensor location of the coastal boundary and is used for the error distance calculations.

This algorithm is based on the notion that the maximum slope location is found where the -3 dB IFOV encompasses half land and half water (50\% beam-fill fraction). This assumption was confirmed by convolving a one-dimensional MWR antenna elevation pattern with a Tb step function representing the land/water boundary, as seen in Fig. 3-11. However, the step size of this convolution was much smaller than the actual MWR sample distance. The following 
example demonstrates the importance of utilizing a parabolic fit to account for this maximum slope location difference when performing distance calculations.

As displayed earlier, two patterns were convolved with the step function: a Gaussian distribution, and the true antenna pattern. Slight variations were observed, but overall they produced the boundary at the same location. The simulated antenna pattern used for Fig. 3-11 has a Gaussian shape with the same half-power beamwidth as the MWR antenna beams. The top panel displays the step function, brightness temperature that the boresight of the MWR sample views, with a sample distance of $\sim 13 \mathrm{~km}$. Convolution of MWR elevation angle with the step function produces the middle panel. Finally, in the bottom panel, the blue line and points represent the MWR samples' slope values and the red line is the parabolic fit to the MWR slope curve. Note that the parabolic fit places the boundary correctly (in between the MWR observations) that is used as the land/water boundary when calculating geolocation error. Were the parabola not used, the maximum slope location would have been displaced by $6.5 \mathrm{~km}$. This magnitude of error is unacceptable. The parabolic fit must be used for error distance calculations. 


\section{Step Function ( $K$ )}
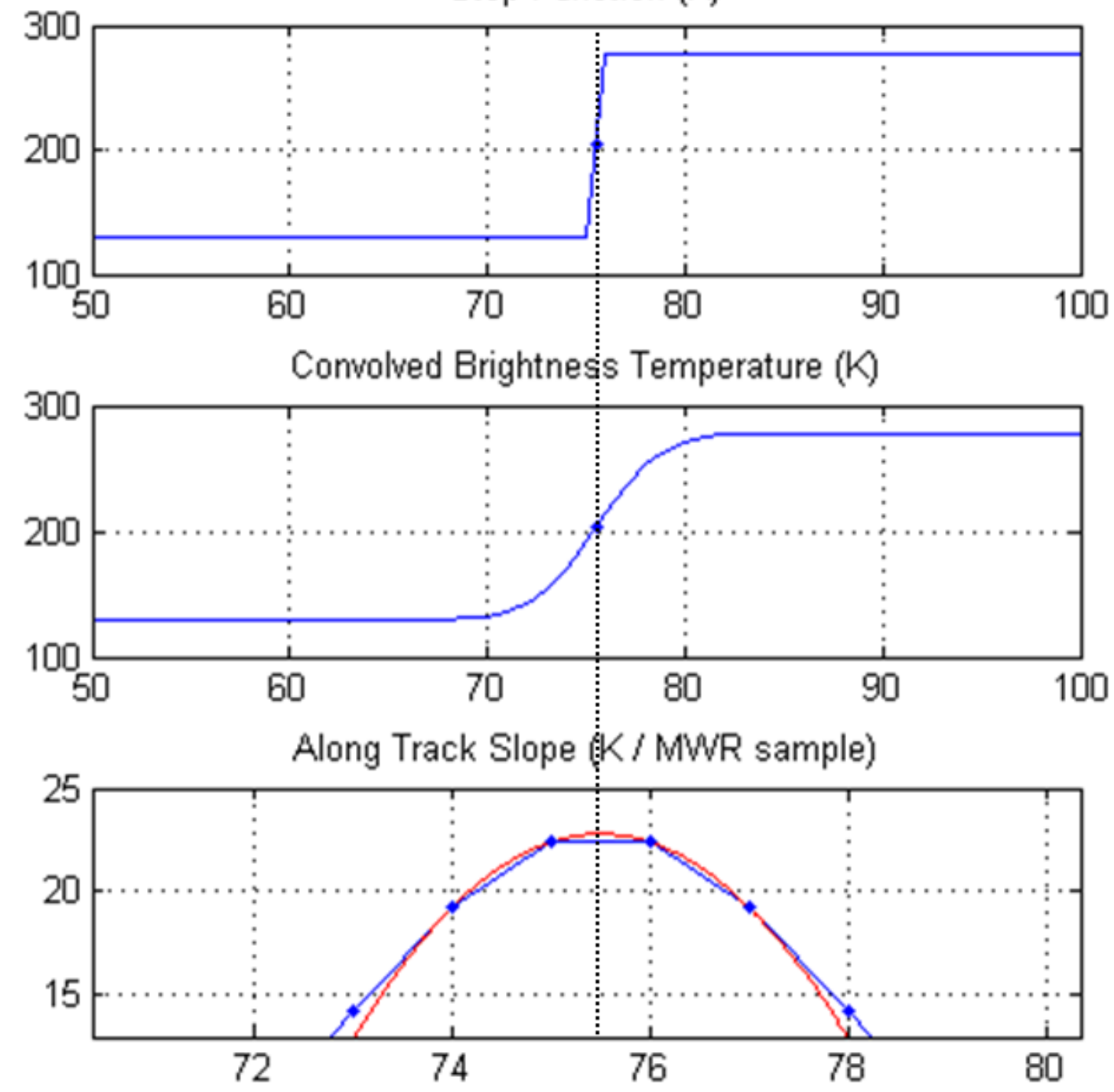

Figure 3-11 One dimensional convolution of antenna pattern with land/water Tb step function. Maximum Tb slope occurs halfway between pixel 75 and 76. 
Since the true MWR sample distance between pixels is $\sim 13 \mathrm{~km}$, the maximum slope location may not occur precisely at this land/water boundary and thereby a random quantization error of $\pm 6.5 \mathrm{~km}$ will be introduced. Applying this parabolic fit to MWR pixels near the maximum Tb slope significantly reduces this quantization error.

An example of the MWR observation parabolic fit to the beam 3, 37V, West Africa coastal crossing is given in Fig. 3-12. The green line defines the brightness temperature for the 7 MWR samples which met the threshold criteria, the blue line is the corresponding slope values for each pixel, and the red line is the parabolic fit to the maximum slope sample and its two leading and lagging pixels. The maximum point of the parabola occurs slightly before the MWR sampled maximum leading to a shift of approximately $4 \mathrm{~km}$ along the MWR track.

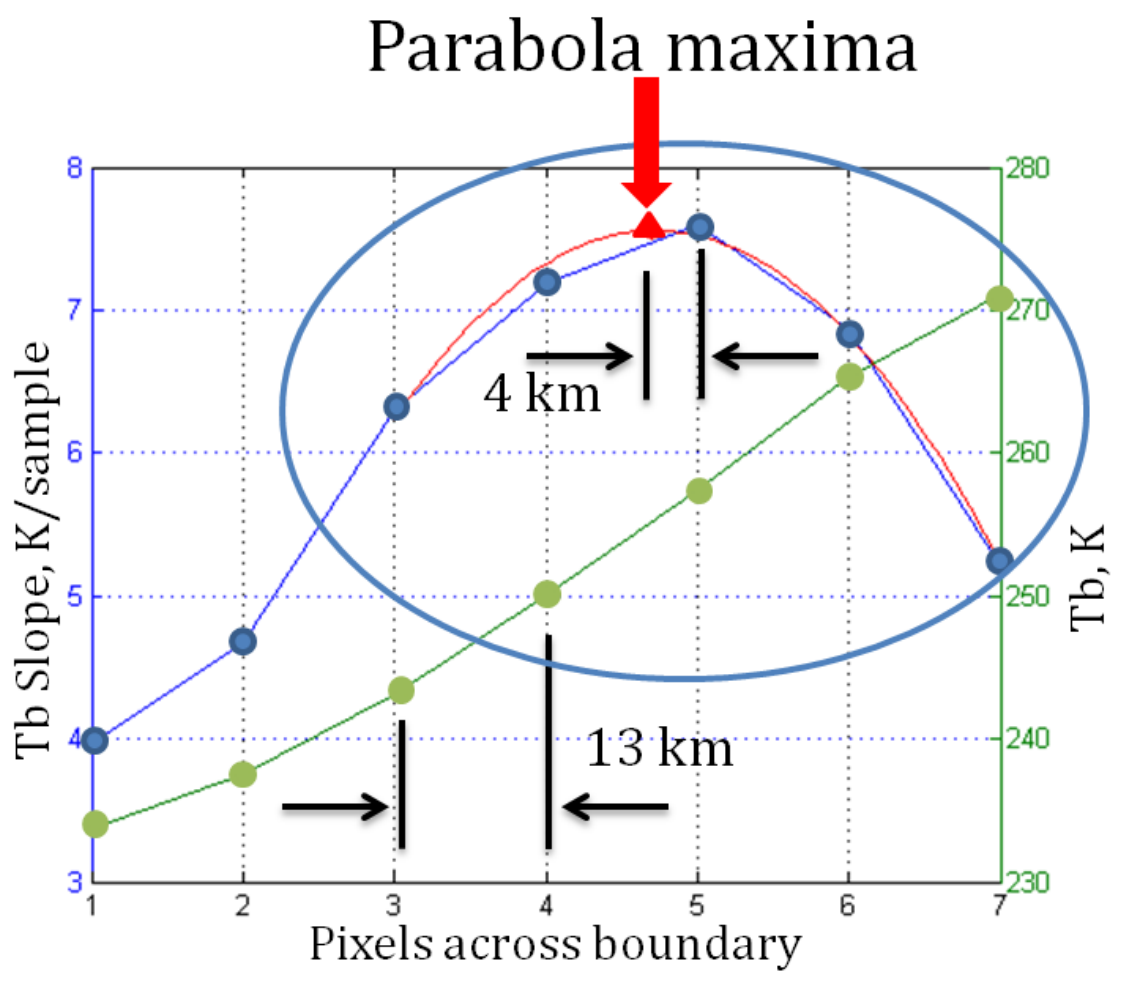

Figure 3-12 Parabolic fit to determine absolute Tb slope maximum 
The new location of the absolute maximum is shown in Fig. 3-13 where the red circle is the MWR maximum Tb slope point and the blue triangle is the parabolic fit maximum slope location. The colors are reversed from Fig. 3-12 because the colors in Fig. 3-13 indicate whether the location is seen before the coastline, or after the coastline. This will be discussed further in an upcoming section. Before the parabolic fit was applied, an error distance calculation of approximately $+4 \mathrm{~km}$ was produced; however, when the location of the new maximum slope from the parabolic fit is used, an improved estimate of the "true" distance from the sensor observed boundary to the coastline is obtained. The new error distance is reduced significantly to less than $1 \mathrm{~km}$. It is necessary that this parabolic fit be applied to account for the quantization error associated with MWR sampling distances.

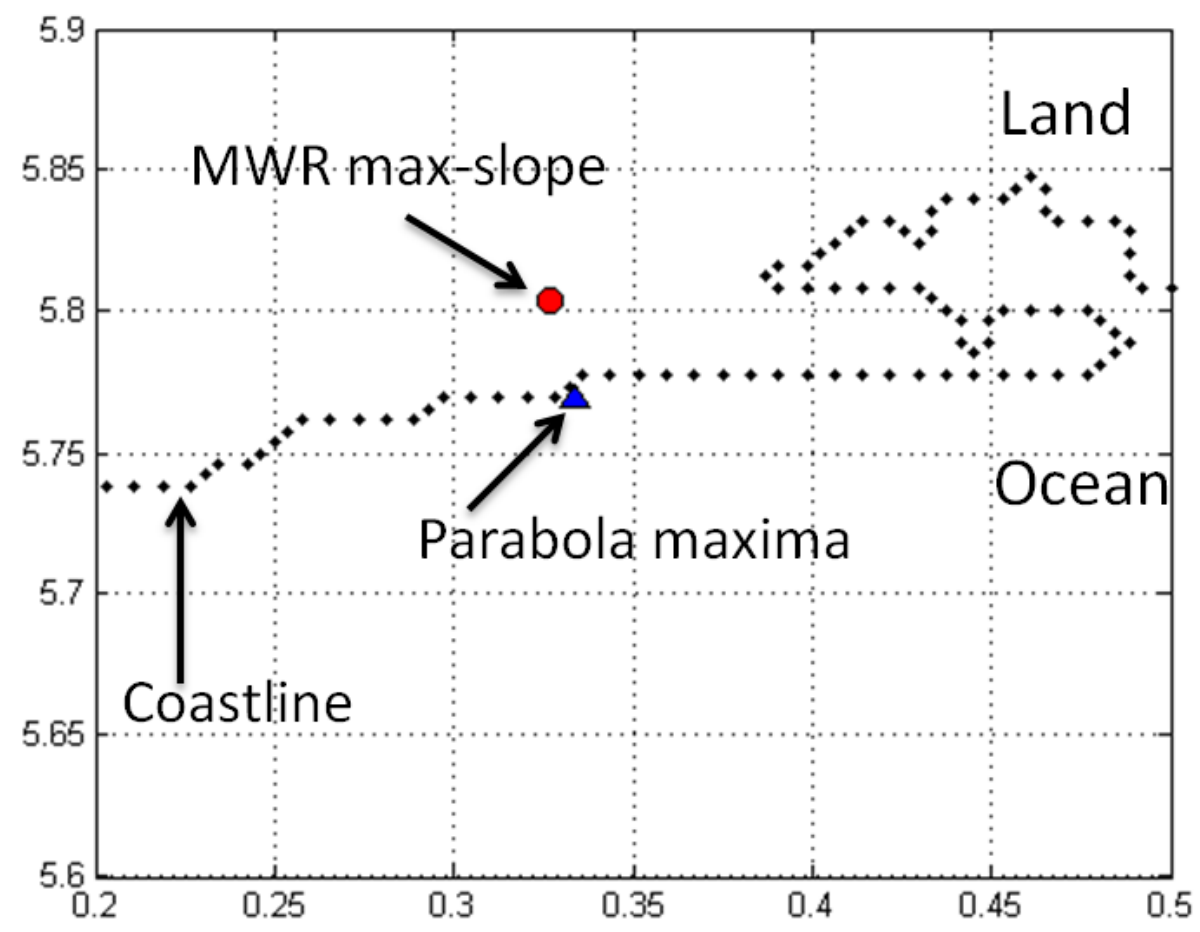

Figure 3-13 Comparison of MWR and parabolic fit, land to water maximum slope (red circleMWR observed boundary, blue triangle - parabolic max) 


\subsubsection{Assigning Latitude and Longitude to the parabolic maximum slope}

The original MWR sensor observed boundary, the two previous, and two lagging pixels are currently used for the parabolic fit. It is fit along the MWR track dimension. Direction and magnitude of the maximum shift must be applied to the original maximum location. Since a vector can always be expressed in terms of its components, the shift of maximum slope along the MWR track can be calculated in terms of latitude and longitude. Spherical coordinates are not necessary for this small shift. This can be characterized in terms of a planar surface. A delta latitude and longitude is determined based on the surrounding MWR pixels from the maximum parabola point. The delta distance along the MWR track from the parabolic maximum location shift can be thought of as the hypotenuse to a right triangle with delta longitude as the change in $\mathrm{x}$ coordinate and delta latitude as change in $\mathrm{y}$. When we have these three distances along with the fraction of change in the hypotenuse distance we can obtain the final delta latitude and longitude with Pythagorean's theorem. To obtain the new parabolic fit boundary, these delta values are applied to the original MWR sensor observed boundary location. This location is used for the sensor boundary error distance calculations.

\subsubsection{Linear Equation Calculations}

Next, the algorithm finds the distance from the absolute maximum slope point to the 1 km resolution map land boundary [12], and for this calculation, two linear equations must be formulated. The first is the locus of the MWR beam boresight intersection with the earth. This "IFOV ground track" equation is calculated using the MWR IFOV center locations surrounding maximum slope location during each crossing, as seen in Fig. 3-14. For this equation, we use a "flat-earth" approximation by constructing a plane tangent to the maximum slope point. Next, we 
use the leading and lagging pixel, relative to the MWR slope maximum (pixel 'i'), to calculate a slope and offset for the MWR track linear equation, with earth latitude ("l”) and longitude (" $n$ ”) coordinates as:

$$
\boldsymbol{Y}=\left(\frac{l_{i+1}-l_{i-1}}{n_{i+1}-n_{i-1}}\right) \boldsymbol{X}+\left(l_{i+1}-\left(\frac{l_{i+1}-l_{i-1}}{n_{i+1}-n_{i-1}}\right) n_{i+1}\right)
$$

Because the distances on the plane are small $(<50 \mathrm{~km})$ and the curvature of the earth is also small, this equation provides an excellent approximation for the actual MWR IFOV ground track at the boundary location.

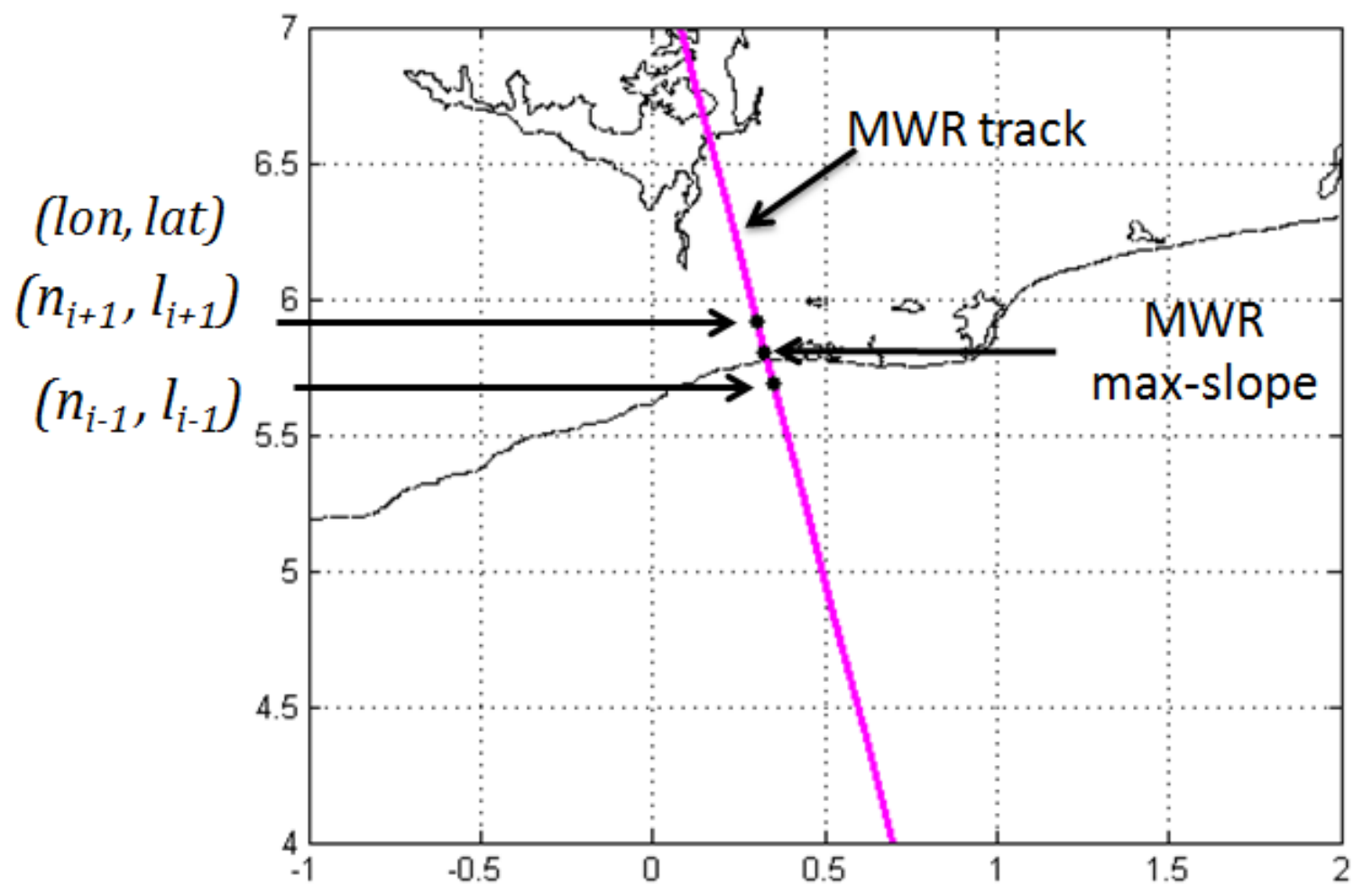

Figure 3-14 MWR track linear equation representation 
The second equation is more difficult to determine as it represents the "coastline", which is calculated using the surrounding pixels of the coastline pixel with minimum distance from the sensor observed land/water boundary. A $\pm 0.1^{\circ}$ latitude by $\pm 0.1^{\circ}$ longitude box of coastline pixels is used to formulate a representative coastline linear equation, defined by the red box in Fig. 315. It is best to fit a regression to many coastal points rather than forming an equation based on the two surrounding coast pixels to the closest pixel, as demonstrated in the MWR track equation. This magenta linear fit is more representative of the overall orientation of the coastline since the one kilometer resolution map can have sharper directional changes due to its improved resolution. Orientation is important for defining angle of intersection, which will be discussed in Chapter 4.

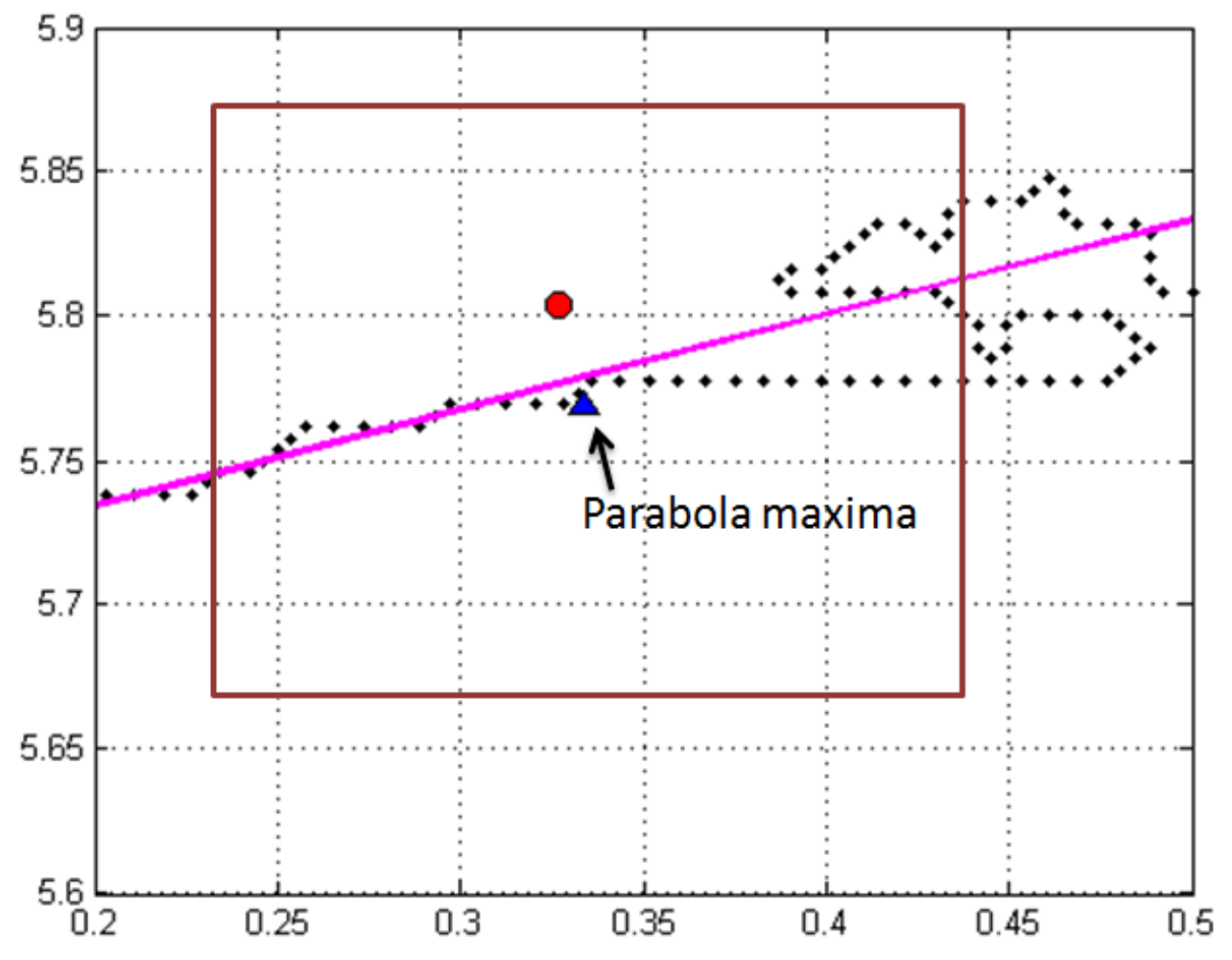

Figure 3-15 Coastline approximation of coastal points within a +/- 0.1 degree box 
The intersection point of the antenna beam ground track with the coastline is found by equating these two linear equations and solving for the mutual latitude and longitude in Fig. 316. This is the exact location where the MWR IFOV should be observing 50\% land and 50\% water. The error distance is calculated from this point, which is assumed to be the true coastal boundary.

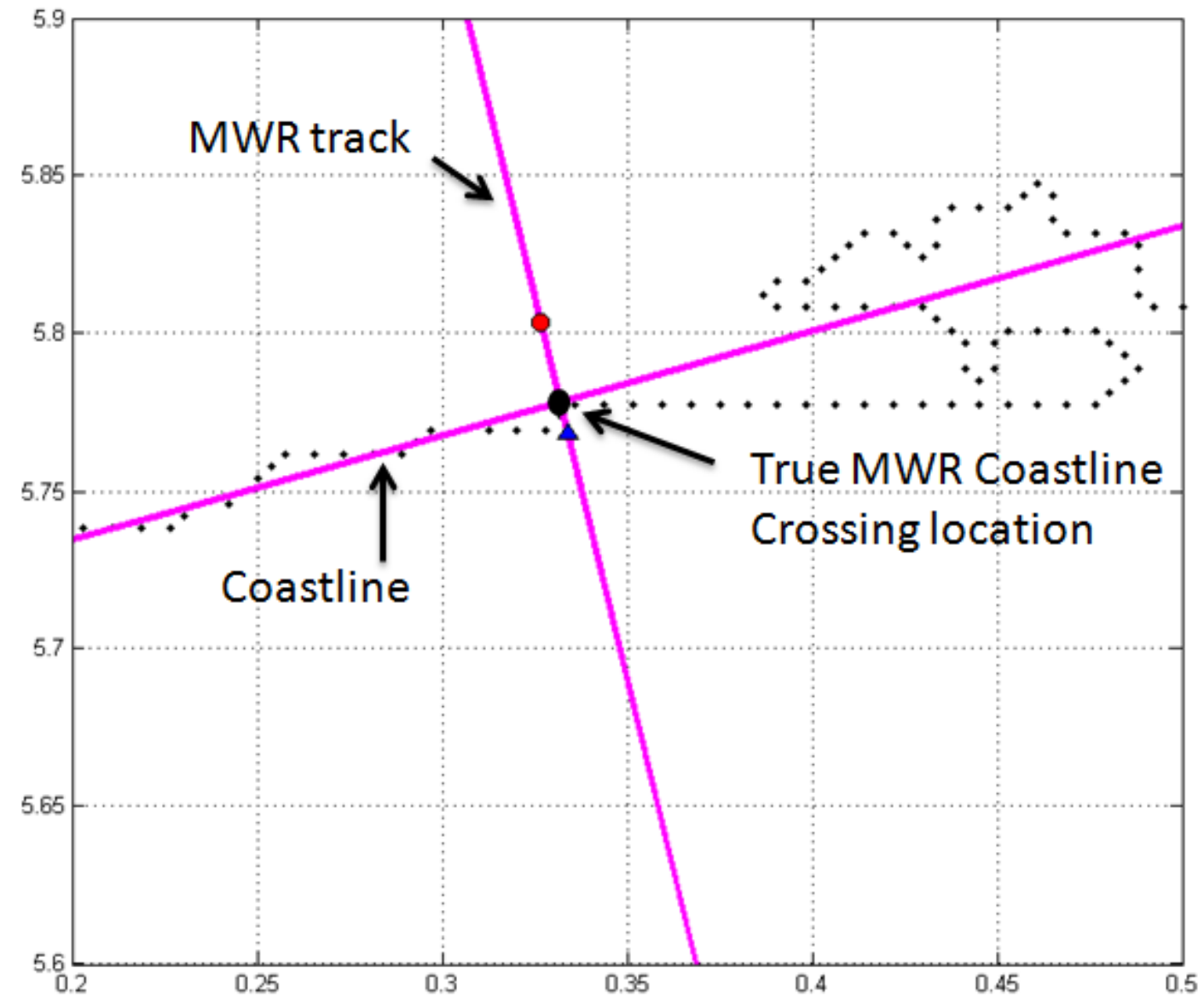

Figure 3-16 Intersection of the two linear equations 


\subsubsection{Error Distance Calculation}

The antenna beam pointing error (in terms of distance on the earth's surface) was performed along the ground tracks of the eight MWR beams by finding the intersection of antenna beam track and coastline, and then calculating the distance between this point of intersection and maximum slope location. Both of these points lie on the MWR antenna beam ground track, and the error distance between the true map coast and the MWR observed coast, in kilometers, is given as;

$$
d=\sqrt{\left[\left(l_{i}-l_{p}\right) 111.12\right]^{2}+\left[\left(n_{i}-n_{p}\right) 111.12 \cos \left(\frac{\left(l_{i}+l_{p}\right)}{2}\right)\right]^{2}}
$$

The subscripts in this error distance equation are as follows: ' $i$ ' is the intersection point of the linear equations and 'p' refers to the maximum slope parabolic fit location. In this equation, the distances are slightly larger than the previous calculations, thus the surface of the earth is assumed spherical to account for changes in longitudinal distance with latitudinal shifts. Therefore, spherical coordinates are used for this calculation. Latitudinal distance is approximated as $111.12 \mathrm{~km}$ per degree latitude and longitudinal distance is a function of latitude, given as $111.12 \mathrm{~km}$ multiplied by the cosine of latitude for every one degree longitude.

As mentioned previously, a positive or negative sign is assigned to each error distance to center the mean error distance about zero. The sign is determined by which distance to the MWR and coastal intersection point is smaller, the leading or lagging pixel of the MWR sensor observed boundary. If the leading pixel is closer to the true coastline, it implies that the sensor is seeing the boundary before it should and a negative value is assigned to the error distance. When 
plotting the errors, positive error values are represented by the color red, while the negative error values are plotted as blue.

This error distance calculation should be precise. Many preemptive measures have been taken to prevent other extraneous errors leading to inaccurate results. Some of them are discussed in the following section.

\subsection{Explanation and Correction of Errors}

Many errors can be encountered when applying this algorithm. Some are attributed to the imperfect brightness temperature 'step’ at a land/water boundary, the boresight never exactly intercepting the boundary, and the coastal map resolution. While all of these errors cannot be perfectly resolved, they can be minimized.

\subsubsection{Transition Error}

If a boundary is a perfect step function, then the maximum slope location is exactly where the $-3 \mathrm{~dB}$ beam footprint encompasses $50 \%$ water and $50 \%$ land. Unfortunately, the $\mathrm{Tb}$ transition from water to land can be drawn-out over many kilometers making it very difficult to justify that the true boundary is found at the maximum slope location. While we cannot define the effect of swamplands or sandbars on maximum slope location, it can be assumed that the transition error occurs on the same side of the coast consistently (ie. the maximum slope will always either be farther inland than expected or vice versa). For later statistical analysis, only the most well defined transitions will be used. 


\subsubsection{Quantization Error}

Another error that can be accounted for is the MWR spatial sample error, or the error due to quantization. MWR takes observations on earth’s surface approximately every $13.1 \mathrm{~km}$. Many times this causes the antenna boresight to be displaced from the coastline; in effect, MWR will not exactly observe 50\% land and water at each land/water transition. On average, this MWR sample distance quantization could produce a $6.55 \mathrm{~km}$ error. To counteract this error, implement a parabolic fit to the surrounding slope samples and find the location of the maximum of the parabola. We use this value as the best estimate of the true MWR max slope location that is the land/water observed boundary. The parabolic fit must be implemented into the assessment algorithm to ensure that quantization error is minimized. Our way to mitigate this error was discussed previously in this chapter.

\subsubsection{Coastline Error}

For this algorithm, the MWR sensor observed boundary is compared to a coastal map, taken as truth. Errors associated with the sensor boundary have been addressed, but error can also be caused by the map. Suppose that a coastal map has a resolution of $10 \mathrm{~km}$. While this would be considered fairly high resolution for some applications, the expected geolocation errors are expected to be much less than that. As a result, a one kilometer coastal map was provided by Remote Sensing Systems (REMSS) [12] and is used for the algorithm. The error associated with the coastal map is assumed to be less than $1 \mathrm{~km}$.

Another error that can be caused by the coastline in this algorithm is actually caused by

the increased resolution. When the coastline is very fine, many times the edges jut back and forth making the linear definition of the coastline highly difficult to accurately portray. This difficulty 
has been mitigated by fitting a linear regression to approximately 15 coastal points. A regression analysis is performed on this fit and regressions with R-squared values less than 0.6 are considered to be inaccurate coastal representations, and are removed from the geolocation assessment. 


\section{CHAPTER 4: RESULTS AND VALIDATION}

\subsection{Validation of the Process}

The geolocation assessment algorithm was tested extensively before the geolocation statistical analysis was performed. An example of this is given in Fig. 4-1, and results demonstrate that the geolocation assessment algorithm performs as desired. This case is a 23 GHz, beam-8, water-to-land crossing off the southeastern coast of Yemen. Note that the symbols plotted are the IFOV center locations, not the entire $-3 \mathrm{~dB}$ footprint. All of the plotted pixels are MWR measurements that meet the threshold criterion of Tb slopes greater than $7 \mathrm{~K} /$ sample and are color coded to indicate the slope magnitude. The maximum MWR slope measurement is represented by the black triangle, but the best estimate of the land/water boundary location was derived from the vertex of the parabolic curve fit to the surrounding slope pixels. This location is plotted as the open black circle.

In Fig. 4-2, the MWR track (magenta line) and the coastline (cyan) linear equations were generated and equated producing the true coastline intersection point represented by the filled black circle. The geolocation error is the distance between the maximum slope point and this intersection. This example results in a negative error distance because the coastline is observed by MWR before map coastline. 


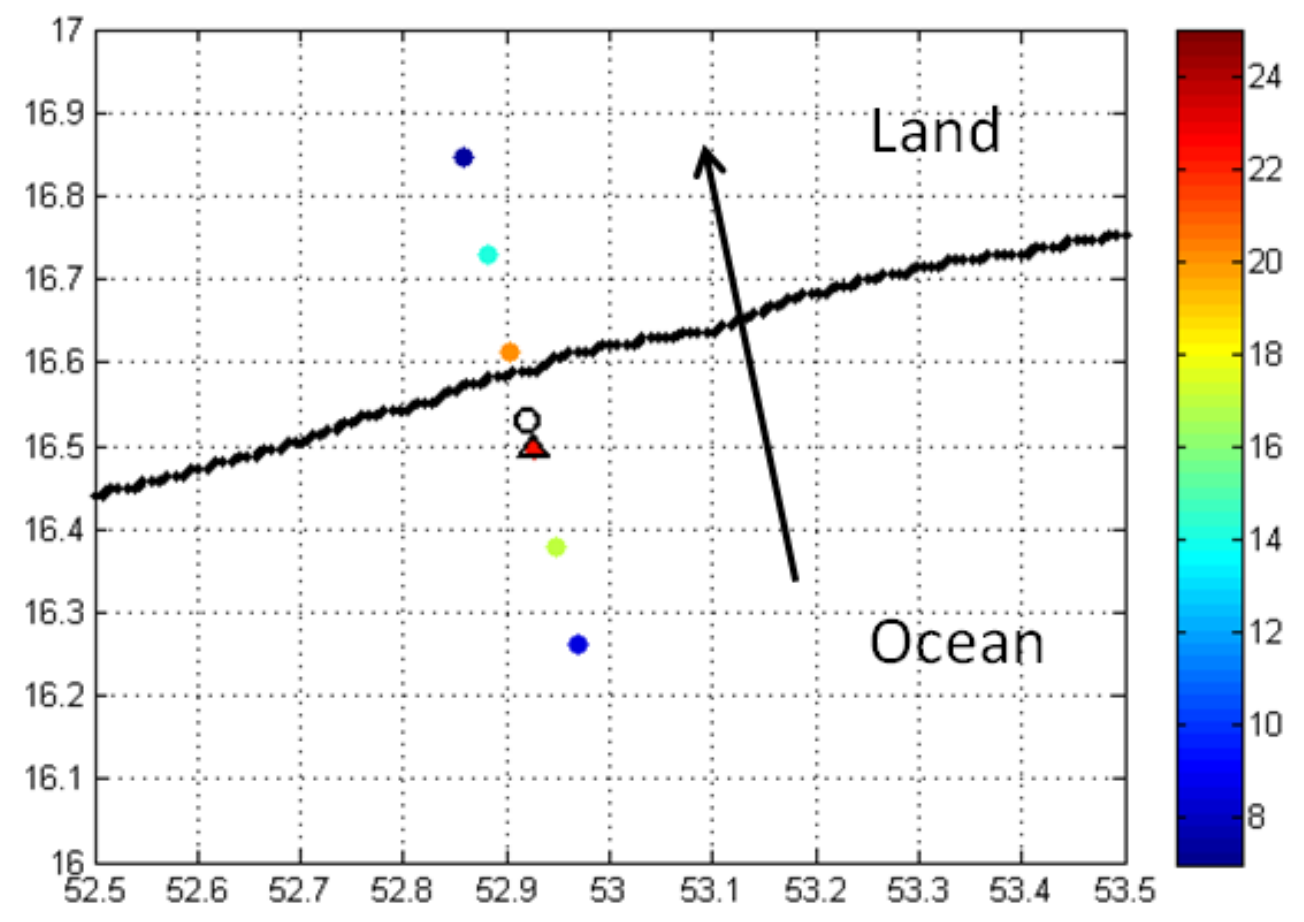

Figure 4-1 Slope value for MWR pixels (absolute maximum indicated as open circle)

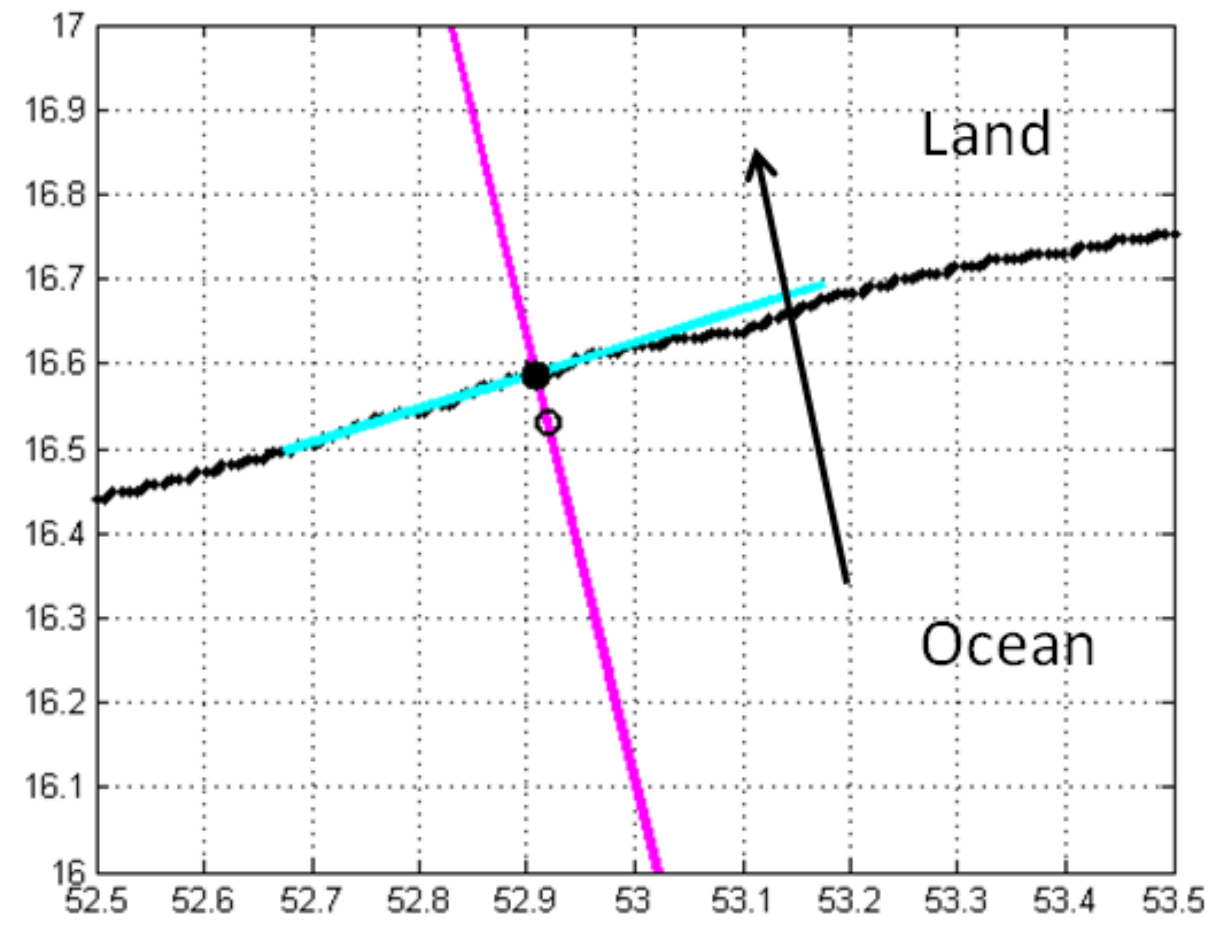

Figure 4-2 Linear equations and intersection point (error distance is calculated from open circle to closed circle) 


\subsubsection{Coastal Boundaries}

Figures $4-2 \mathrm{a}-\mathrm{b}$ are overlays of all $(23 \mathrm{H}, 37 \mathrm{~V} \& \mathrm{H})$ sensor observed land/water crossings on a coastal maps of the northeastern United States for a 7-day (103 orbits) period, where ascending crossings are plotted in red and descending are plotted in blue. Both the Matlab generated coastlines (Fig. 4-3) and the one-kilometer coastal map (Fig. 4-4) are plotted to emphasize the importance of a high resolution map in this algorithm. Lakes that are detected by MWR using the maximum slope criterion will be excluded from the algorithm statistics when using the low resolution map because the geolocation error distance is $>50 \mathrm{~km}$. By using the high resolution map, we are able to include many of the MWR land/water crossings that occur for lakes.

It is impressive how well the algorithm is able to differentiate boundaries; surprisingly, even the Great Lakes and Canada’s Lac-Saint Jean are observed when using the maximum slope method. This image verifies that the majority of land/water crossings are preserved, while simultaneously preventing numerous outliers. The MWR beam-4 IFOVs of an ascending pass at approximately 40 degrees latitude are plotted to illustrate the size of a $-3 \mathrm{~dB}$ footprint. These beams have an incidence angles of $58^{\circ}$ and relative azimuth angles of $29.6^{\circ}$.

Occasionally anomalous pixels (outliers) were produced by the slope-filter, but they occurred in the ocean (far away from any coastline) or over land (in the presence of lakes that are not included in this coastal map). The algorithm only performs statistics on locations with error distances $<50 \mathrm{~km}$; therefore, these bogus boundary points were excluded from the geolocation error analysis. 


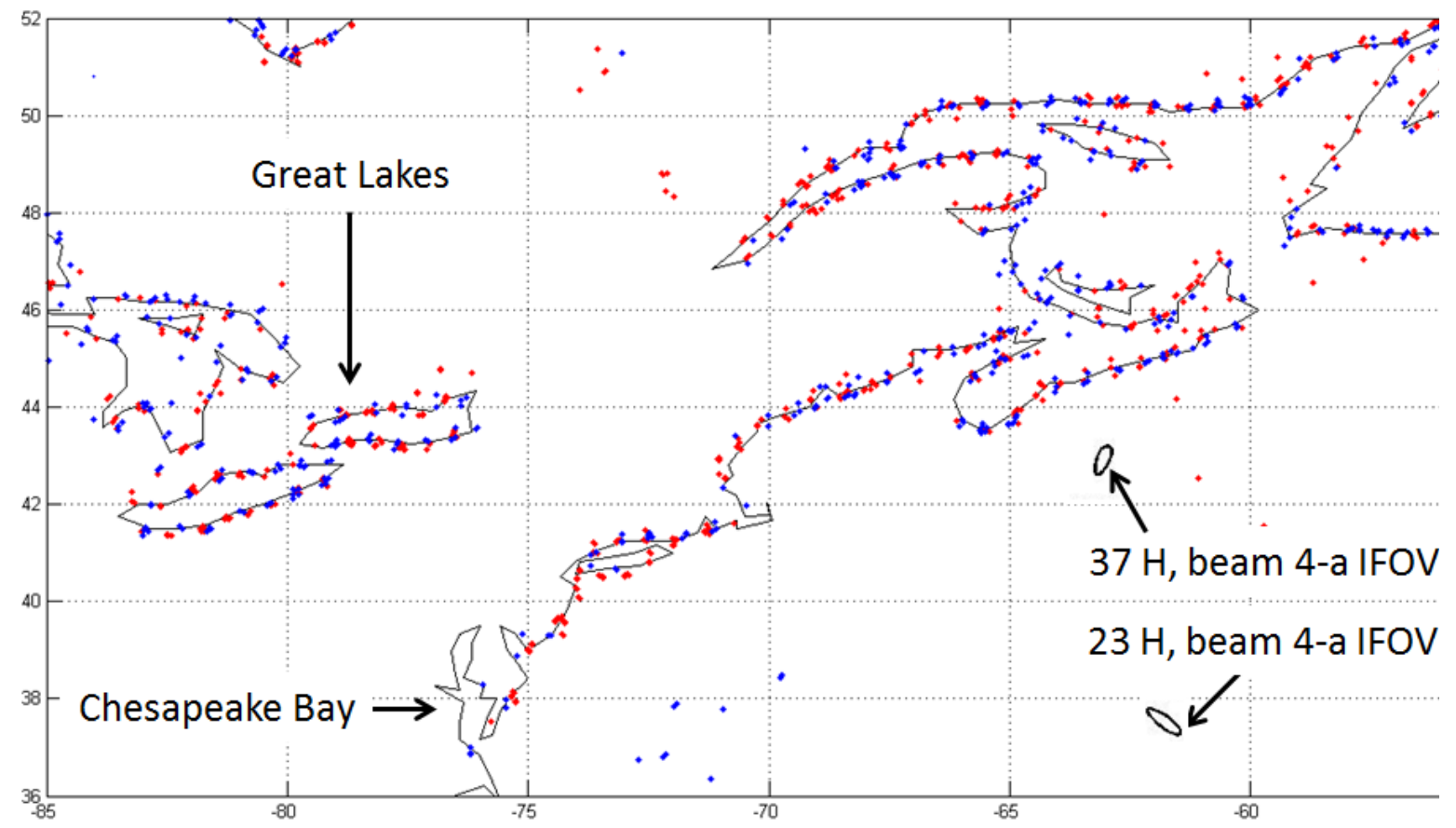

Figure 4-3 MWR sensor observed boundaries and Matlab generated coast (blue-descending, red-ascending) with beam-4 IFOVs shown for an ascending orbit

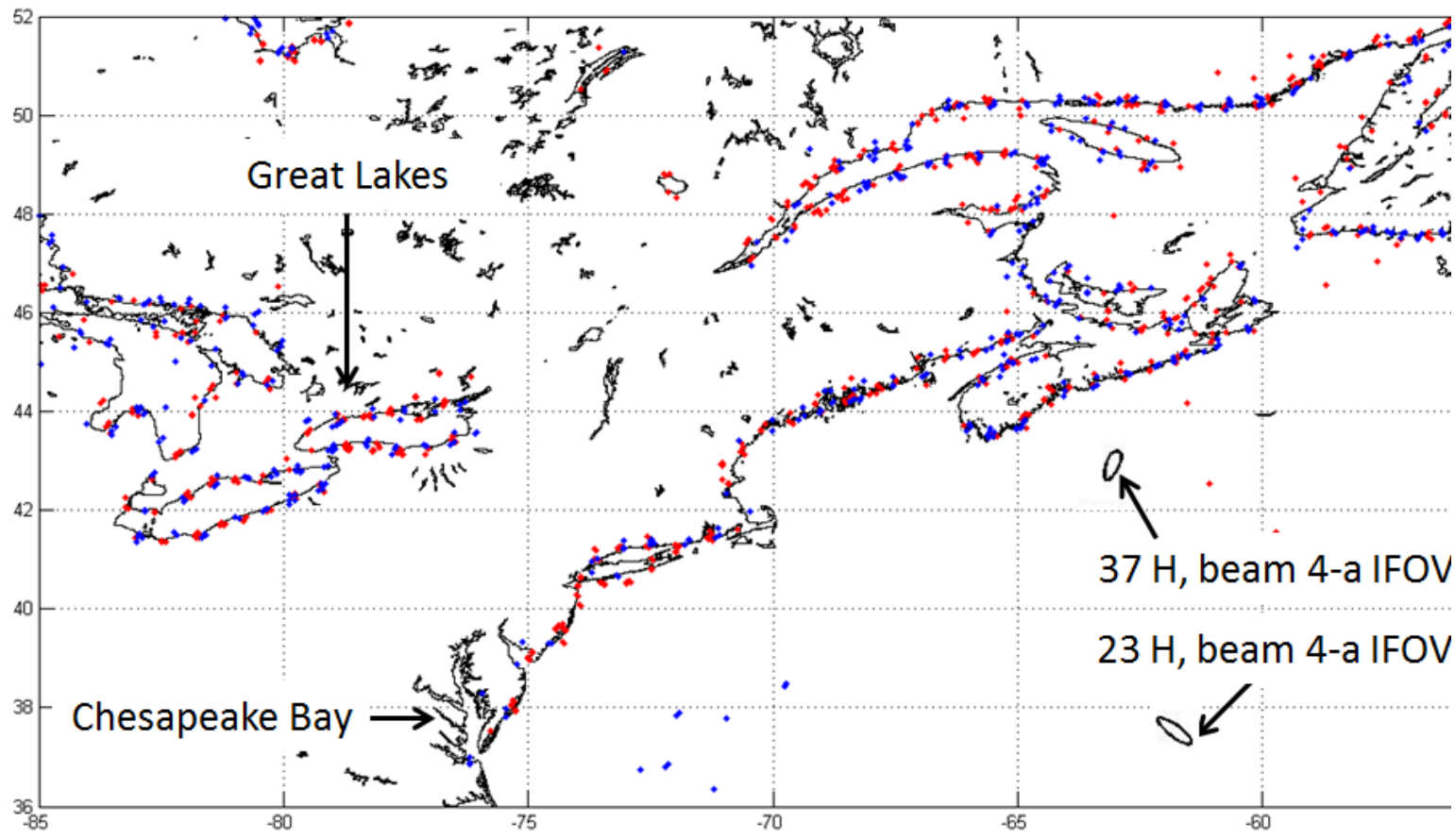

Figure 4-4 MWR sensor observed boundaries and $1 \mathrm{~km}$ resolution coast (blue-descending, redascending) with beam-4 IFOVs shown for an ascending orbit 


\subsubsection{Threshold Validation}

As mentioned previously, the thresholds for determining boundaries were set so each channel produces approximately the same number of crossings. It can be seen from Tables 1-3 that the numbers of crossings are near 800 for each beam of each channel.

The exception is beam-2, which may be caused by higher than normal antenna pattern sidelobes [2]. This sidelobe issue is manifested as a reduction in the Tb slopes at land/water boundaries compared to the other seven beams. While the slope is lower and fewer points meet the threshold, the algorithm still performs reasonably well for this beam, and the geolocation errors are similar to other beams.

Table 1 Number of boundaries used to estimate the error distance statistics for $23 \mathrm{H}$

\begin{tabular}{|l|l|l|l|l|l|l|l|l|l|l|}
\hline Beam \# & $\mathbf{1}$ & $\mathbf{2}$ & $\mathbf{3}$ & $\mathbf{4}$ & $\mathbf{5}$ & $\mathbf{6}$ & $\mathbf{7}$ & $\mathbf{8}$ \\
\hline Asc \# Pixels & 418 & 366 & 478 & 403 & 434 & 466 & 438 & 471 \\
\hline Des \# Pixels & 366 & 329 & 446 & 392 & 437 & 412 & 394 & 416 \\
\hline Total & 784 & 695 & 924 & 795 & 871 & 878 & 832 & 887 \\
\hline
\end{tabular}

Table 2 Number of boundaries used to estimate the error distance statistics for $37 \mathrm{~V}$

\begin{tabular}{|l|l|l|l|l|l|l|l|l|l|l|}
\hline Beam \# & $\mathbf{1}$ & $\mathbf{2}$ & $\mathbf{3}$ & $\mathbf{4}$ & $\mathbf{5}$ & $\mathbf{6}$ & $\mathbf{7}$ & $\mathbf{8}$ \\
\hline Asc \# Pixels & 431 & 273 & 424 & 338 & 447 & 401 & 436 & 357 \\
\hline Des \# Pixels & 346 & 233 & 432 & 312 & 429 & 318 & 389 & 300 \\
\hline Total & 777 & 506 & 856 & 650 & 876 & 719 & 825 & 657 \\
\hline
\end{tabular}

Table 3 Number of boundaries used to estimate the error distance statistics for $37 \mathrm{H}$

\begin{tabular}{|l|l|l|l|l|l|l|l|l|l|l|}
\hline Beam \# & $\mathbf{1}$ & $\mathbf{2}$ & $\mathbf{3}$ & $\mathbf{4}$ & $\mathbf{5}$ & & $\mathbf{6}$ & $\mathbf{7}$ & $\mathbf{8}$ \\
\hline Asc \# Pixels & 446 & 332 & 413 & 389 & 402 & 422 & 373 & 402 \\
\hline Des \# Pixels & 360 & 298 & 426 & 359 & 387 & 389 & 339 & 361 \\
\hline Total & 806 & 630 & 839 & 748 & 789 & 811 & 712 & 763 \\
\hline
\end{tabular}




\subsection{Analysis Results}

The first analysis is performed per beam to compare ascending and descending passes. This will reveal patterns and lead to the next step of analysis, combining beams.

\subsubsection{Ascending and Descending Comparisons}

It is very necessary to classify the pixels as ascending or descending. By analyzing them separately, satellite attitude errors become evident. Chapter 2 described that pitch errors will cause the ascending and descending errors to match, and roll errors are evident when ascending and descending errors span opposite sides of the true boundary.

\subsubsection{Separate Beams}

Each beam was observed separately to determine if error patterns existed for the entire channel, at different incidence angles (even or odd beams), or by beam. Examples of coastline crossing errors for the $23.8 \mathrm{GHz}$ beams for the one-week period of AQ orbits are given below in Figs. 4-5 - 12. In general, these errors were approximately Gaussian distributed, with the majority (> 85\%) of the absolute error distance calculations being less than $20 \mathrm{~km}$. Because the error histograms are Gaussian, the mean and standard deviation are excellent ways to characterize the distribution.

The following plots in Figs. 4-13 - 18 give the geolocation error statistical mean and standard deviation for each beam after removing the bogus outliers. Ascending is plotted in red, and descending in blue. It should be noted that for the $23 \mathrm{GHz}$ channel all of the mean error calculations lie on the negative and relatively small. Initial analysis would show that this pattern is indicative of slight pitch error. Further analysis will be conducted to prove this hypothesis. 
Because the difference between all eight beams' mean error is much less than one standard deviation, all 8 beams can be categorized as one to indicate that an error is not produced by different incidence angles. This will increase the number of points used for later analysis on latitude dependence, angle of intersection, etc.

Also, there seems to be a pattern in the 37V\&H channels. The even beams are producing very small negative errors while the odd beam errors are slightly positive. This pattern allows us to combine all even beams and all odd beams, but does not allow us to combine all eight $37 \mathrm{GHz}$ beams. 


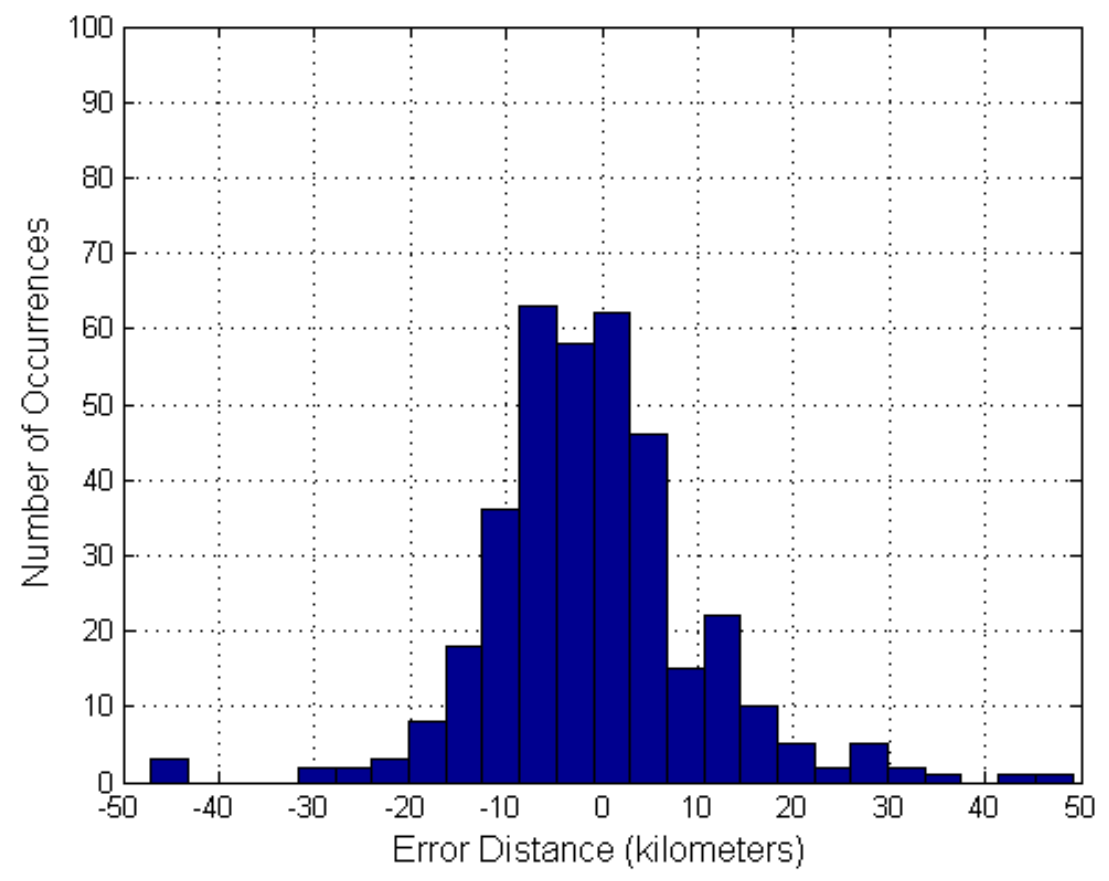

Figure 4-5 Beam 1, 23H channel, descending error distance

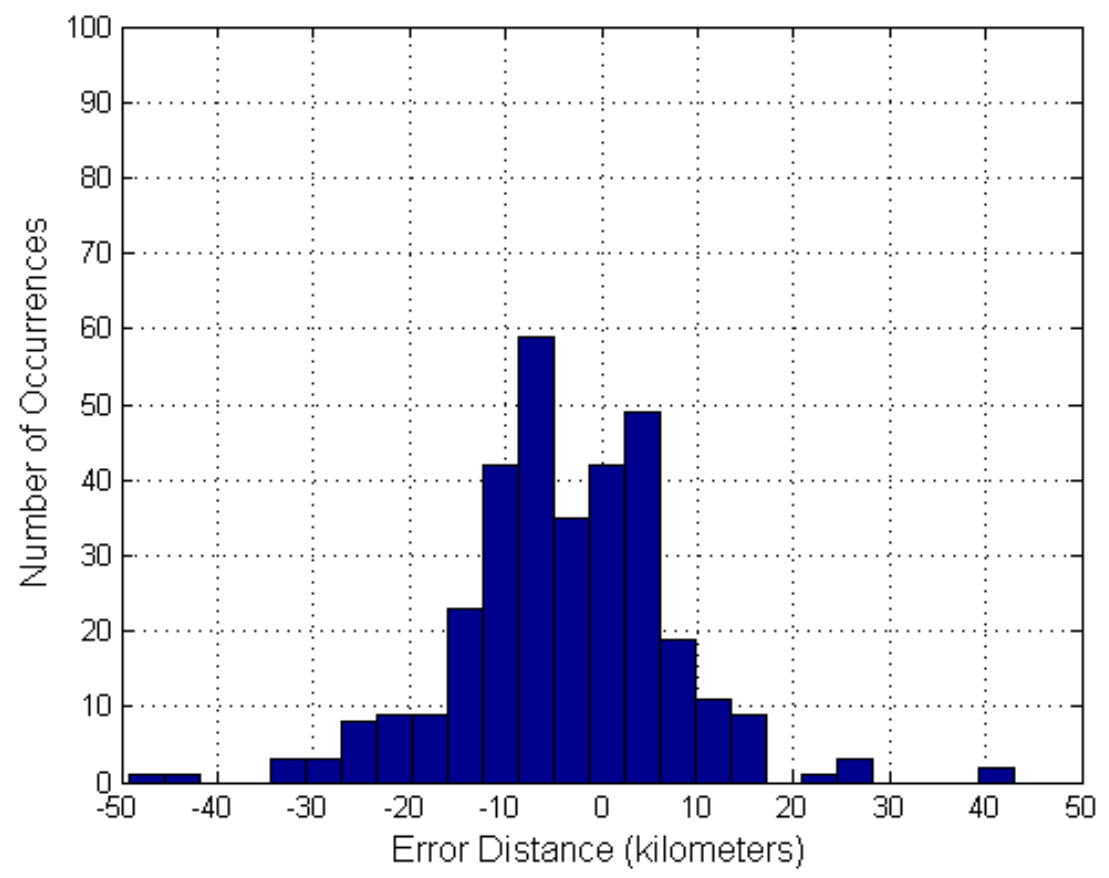

Figure 4-6 Beam 2, 23H channel, descending error distance 


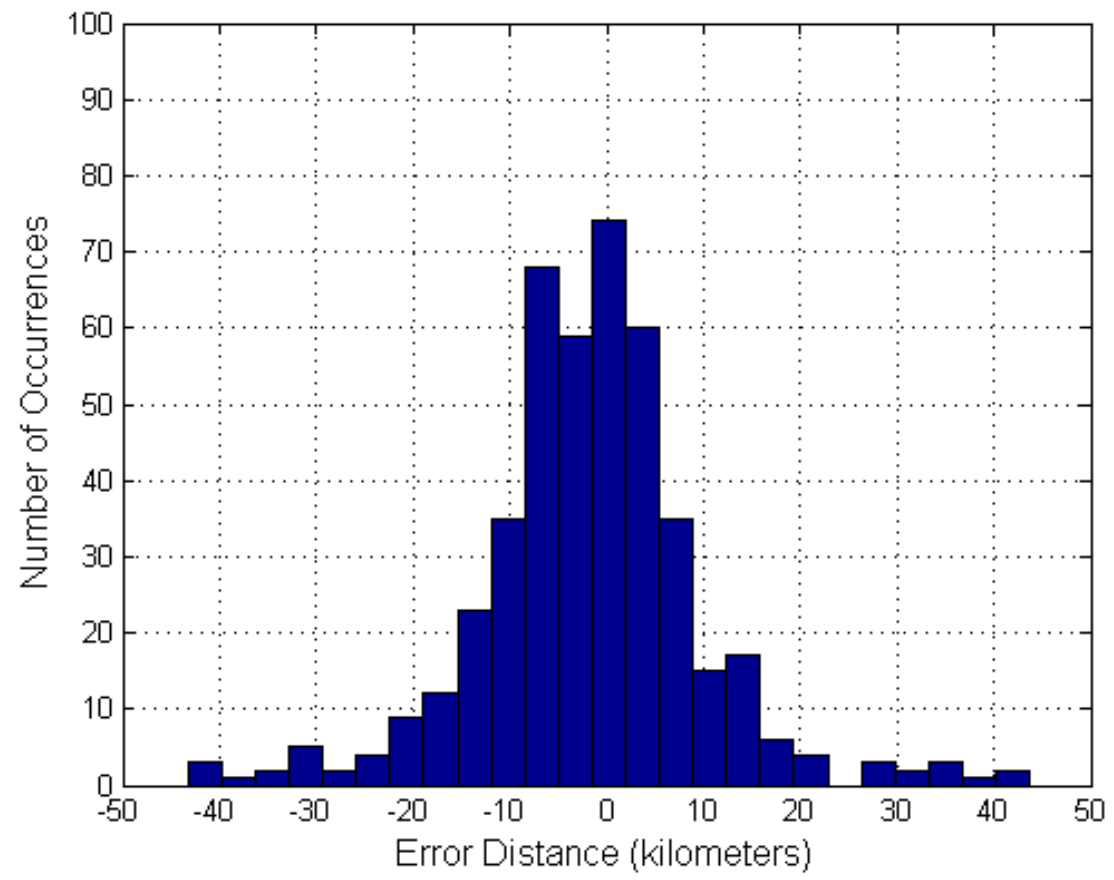

Figure 4-7 Beam 3, 23H channel, descending error distance

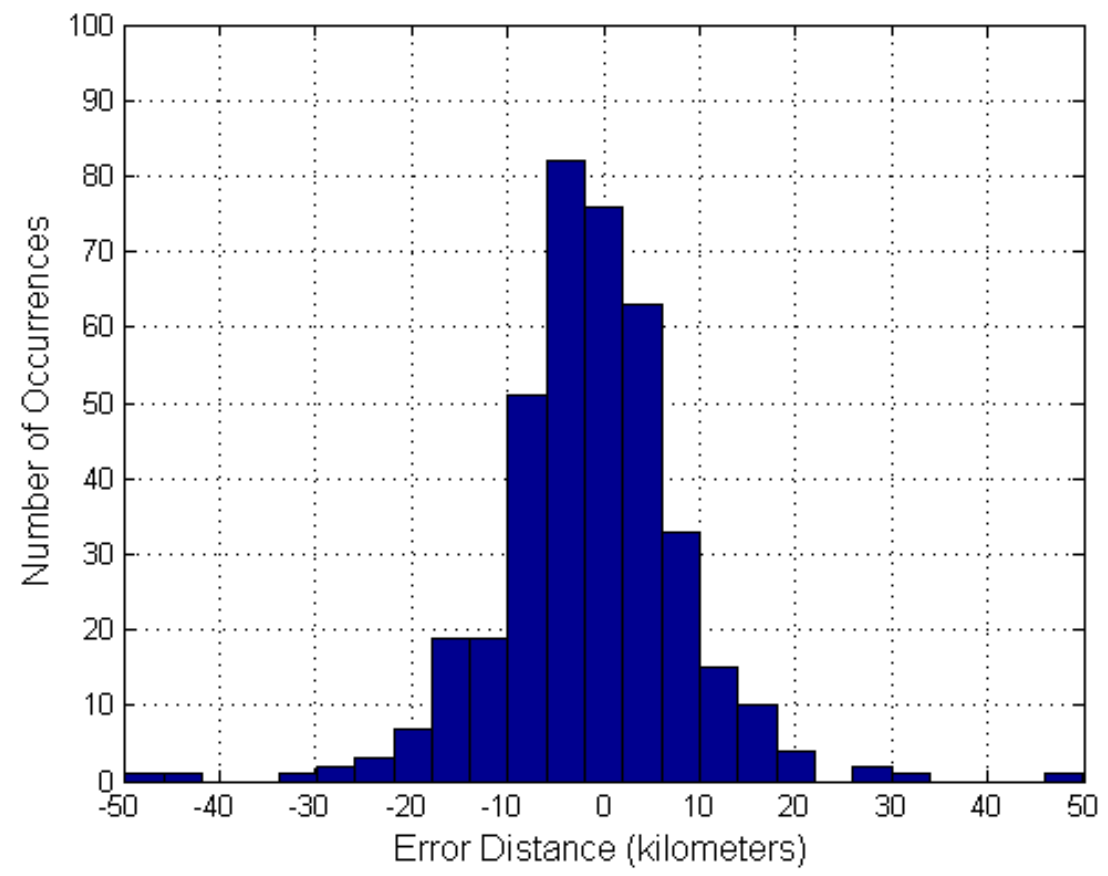

Figure 4-8 Beam 4, 23H channel, descending error distance 


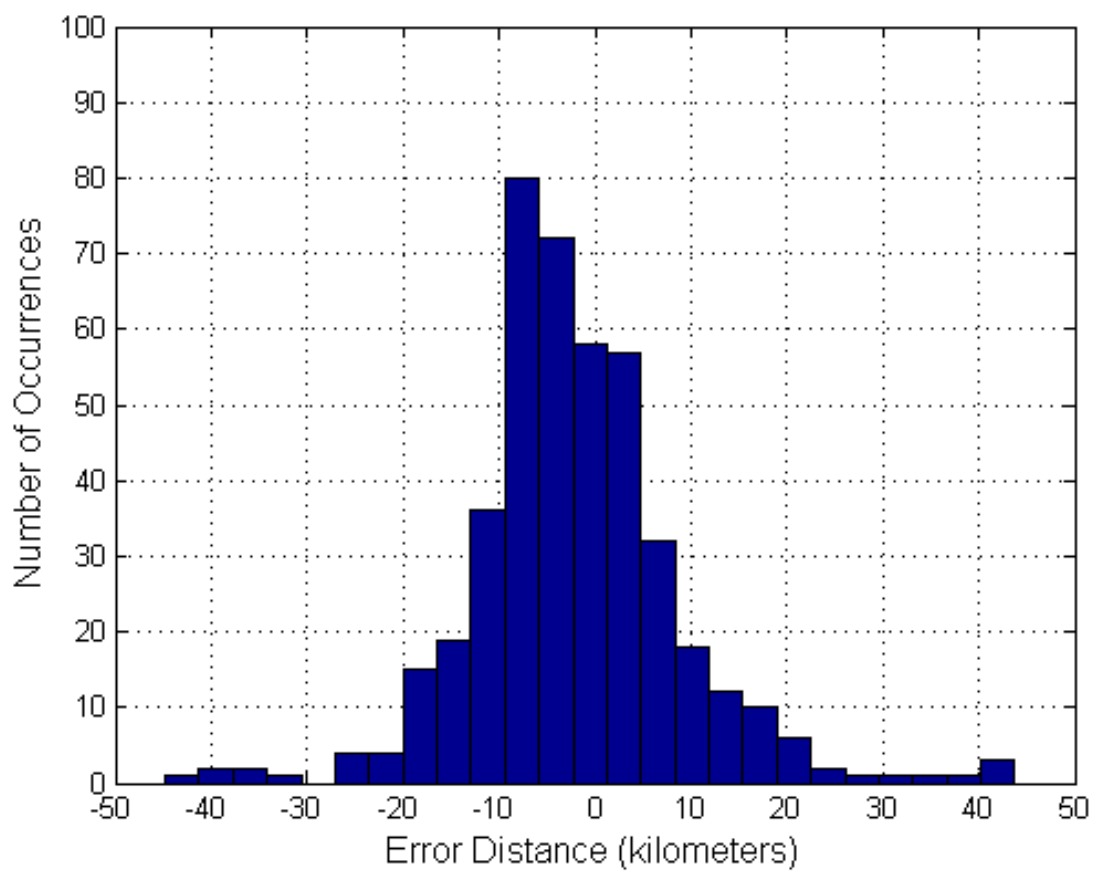

Figure 4-9 Beam 5, 23H channel, descending error distance

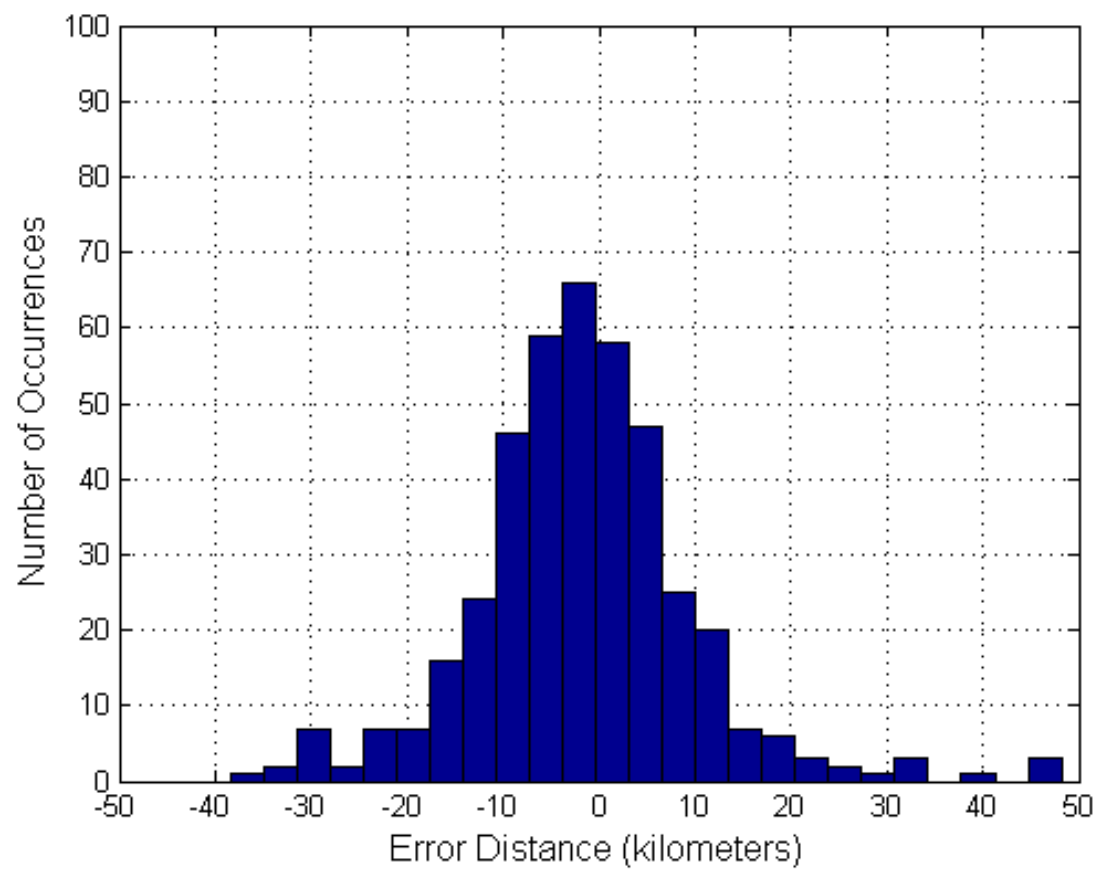

Figure 4-10 Beam 6, 23H channel, descending error distance 


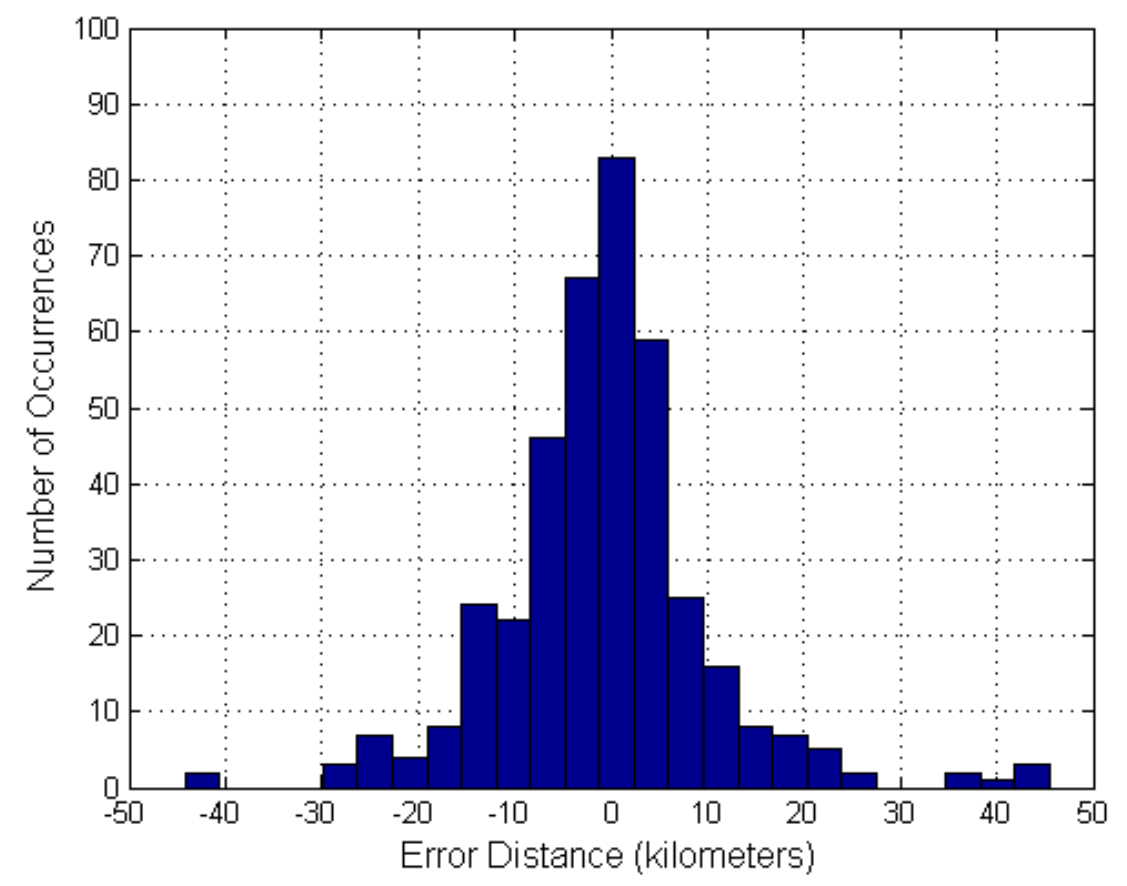

Figure 4-11 Beam 7, 23H channel, descending error distance

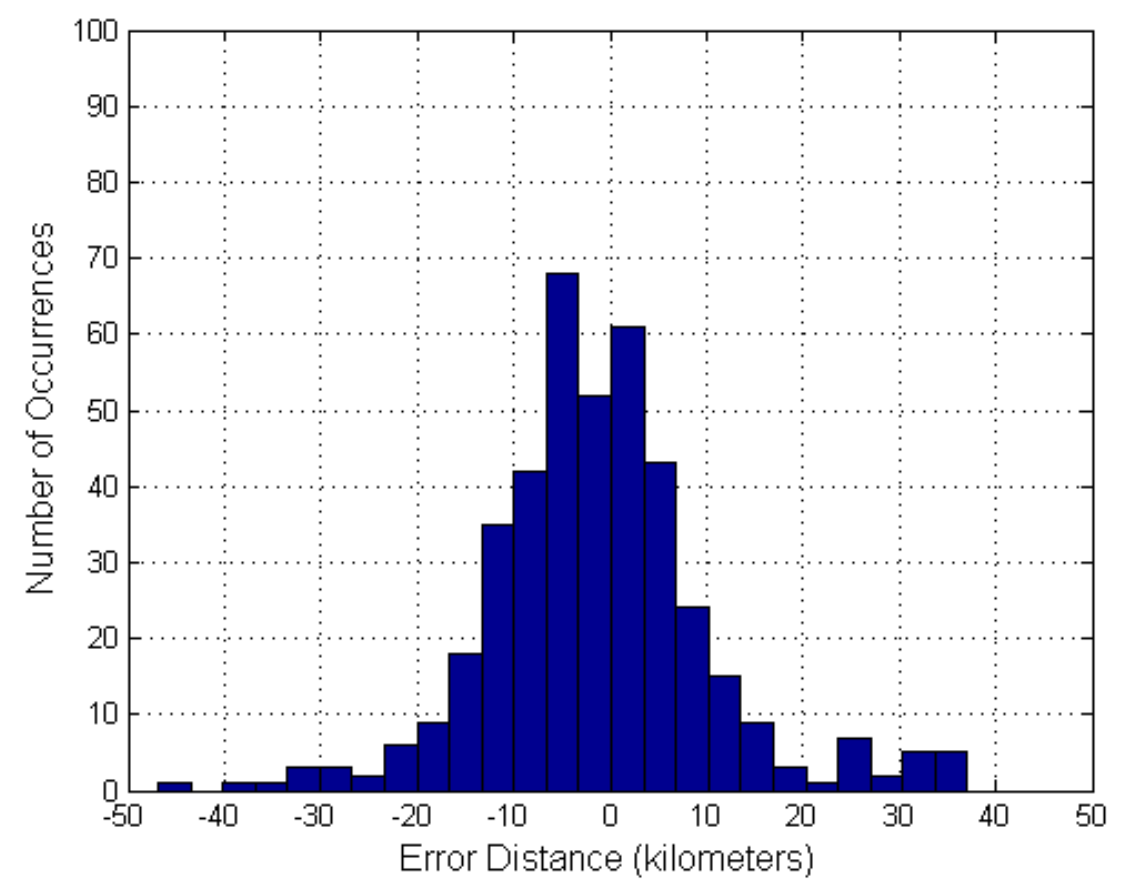

Figure 4-12 Beam 8, 23H channel, descending error distance 


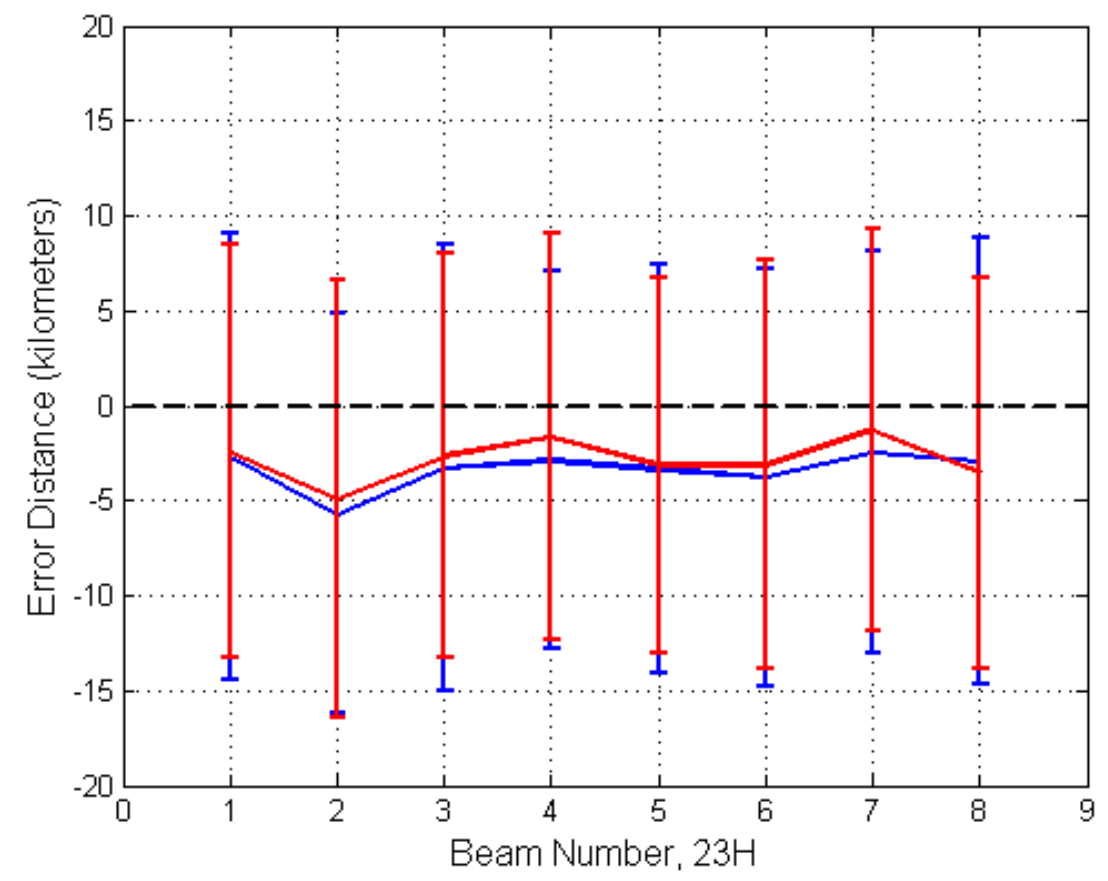

Figure 4-13 Original mean and standard deviation error bars, 23H

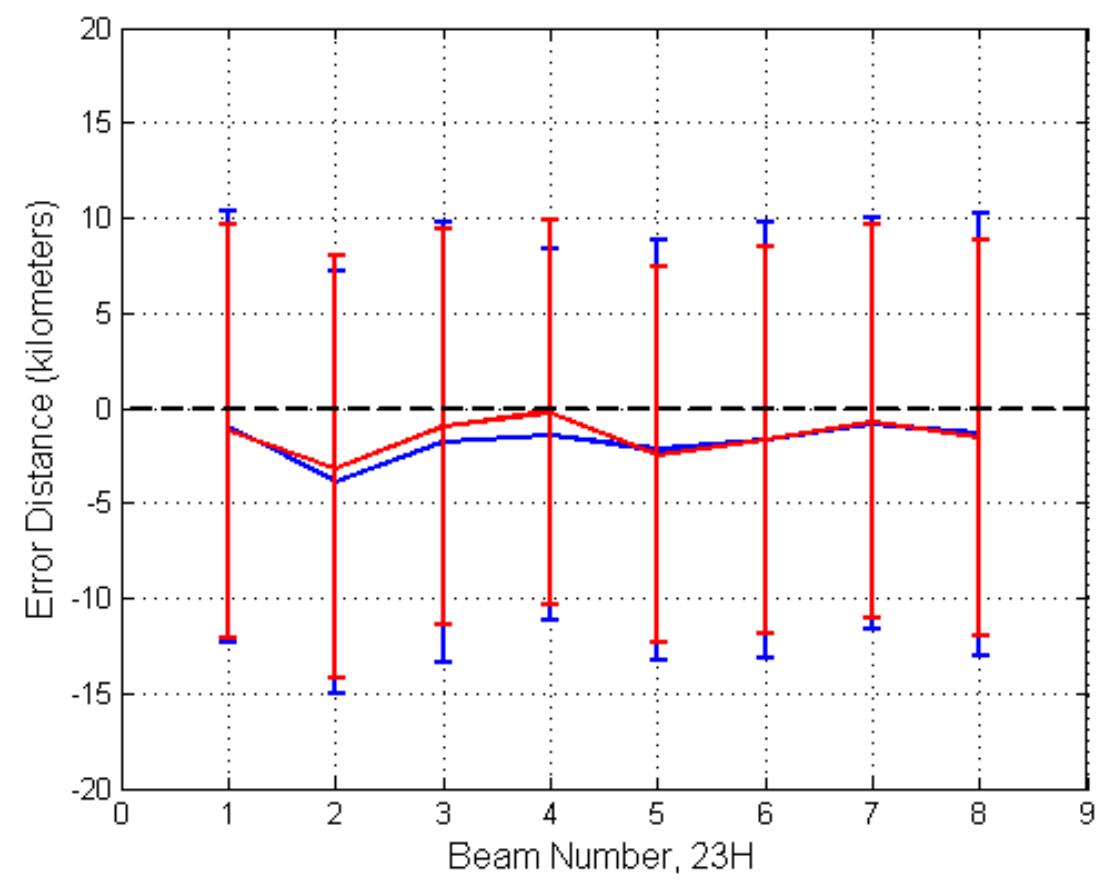

Figure 4-14 Parabolic fit mean and standard deviation error bars, 23H 


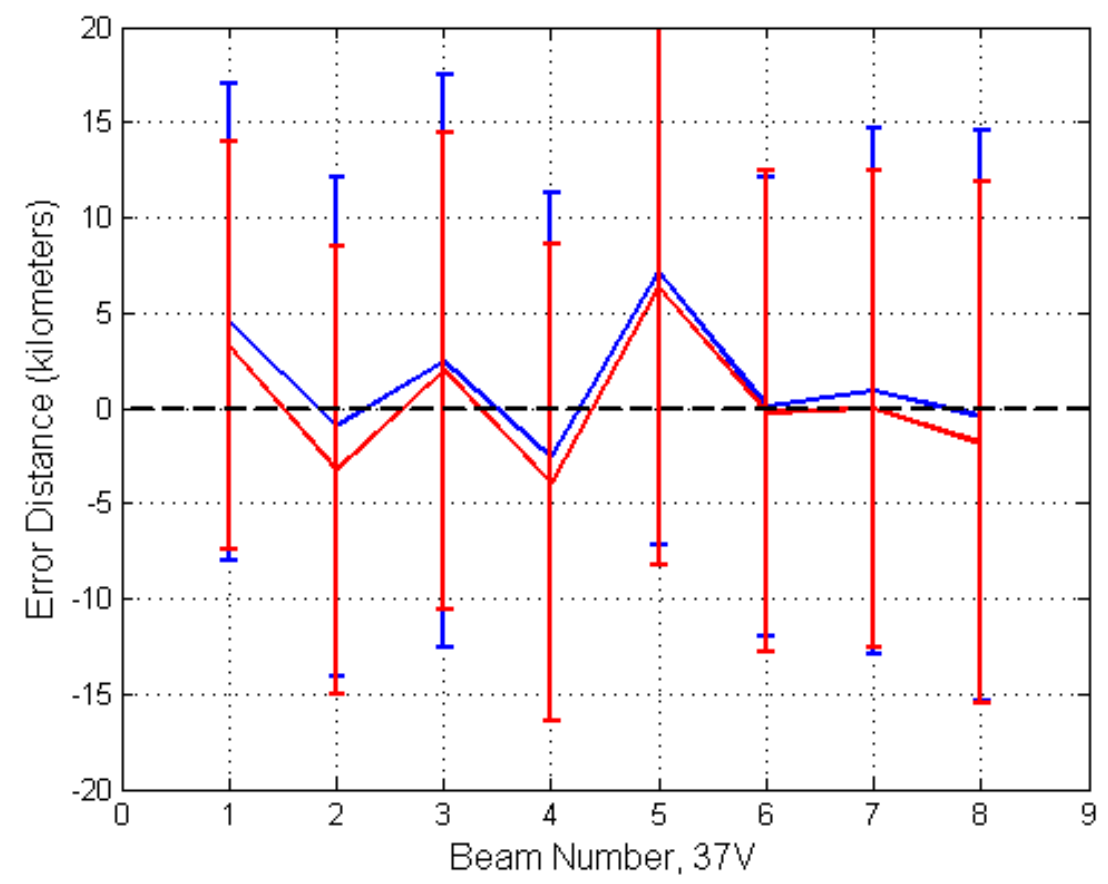

Figure 4-15 Original mean and standard deviation error bars, 37V

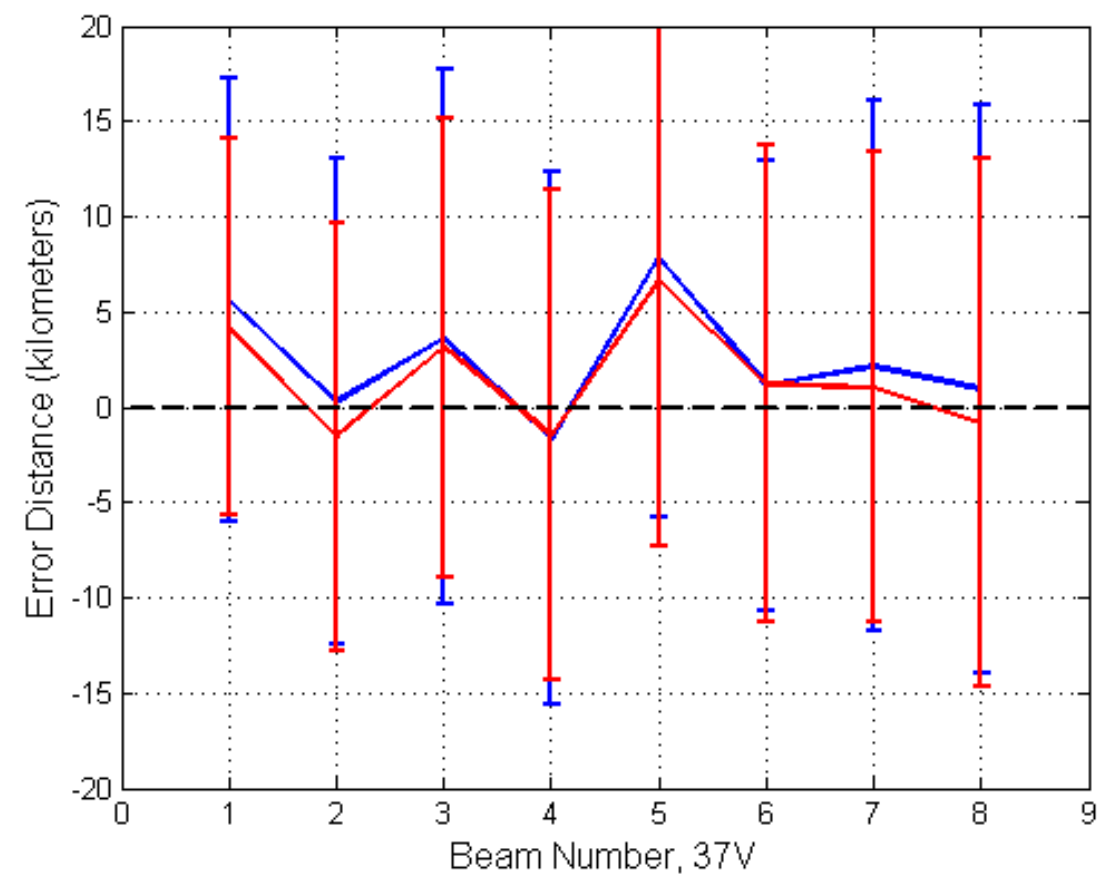

Figure 4-16 Parabolic fit mean and standard deviation error bars, 37V 


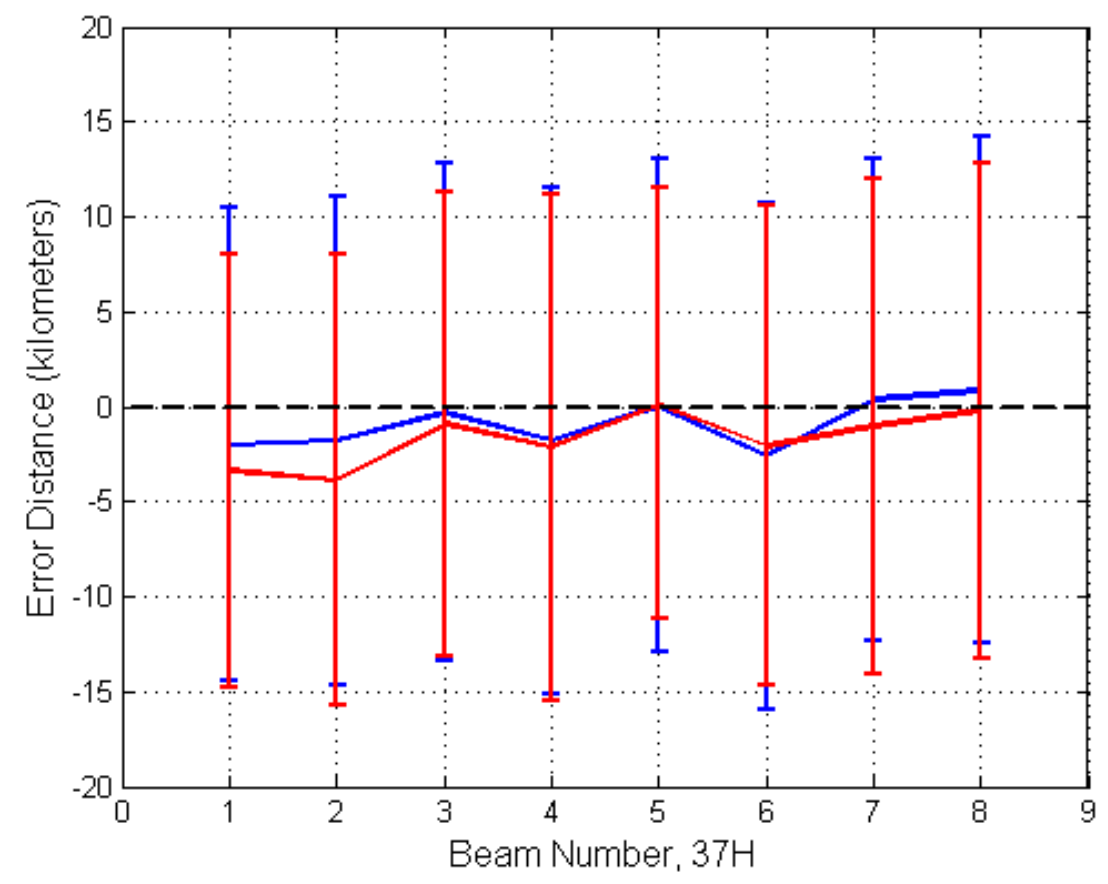

Figure 4-17 Original mean and standard deviation error bars, 37H

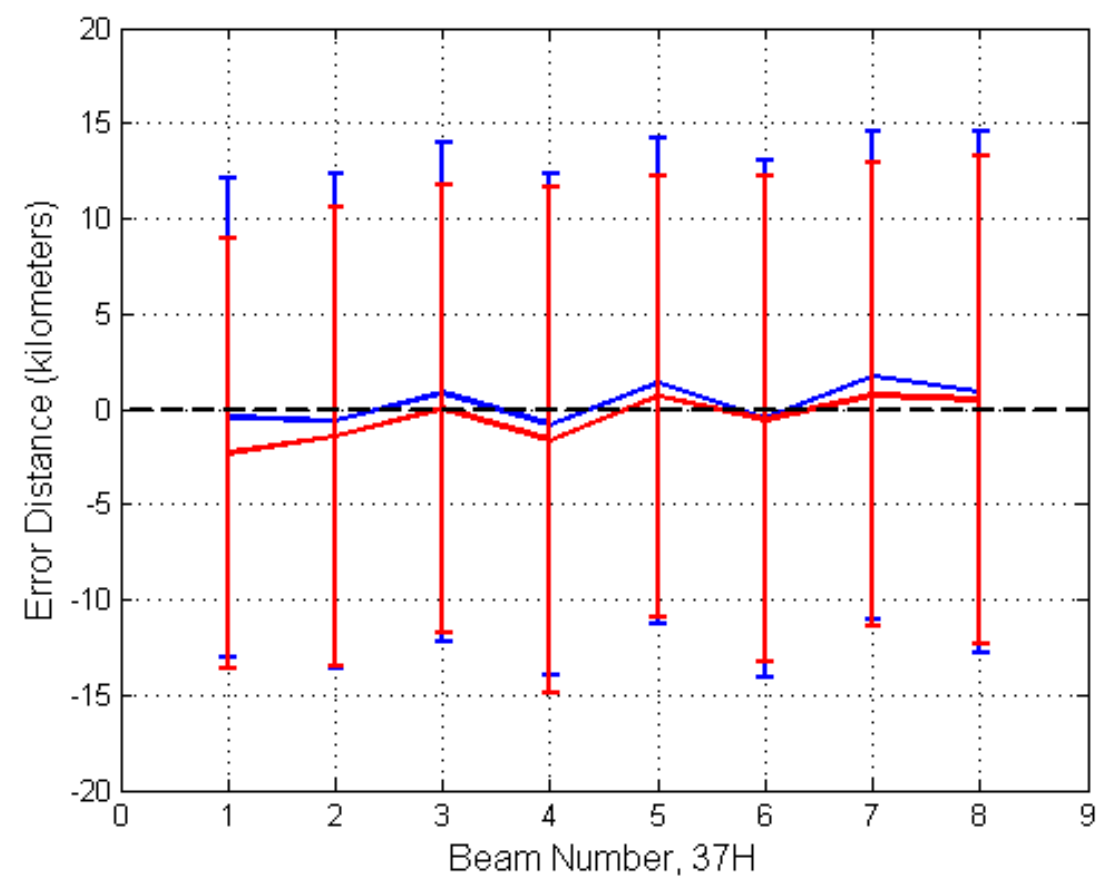

Figure 4-18 Parabolic fit mean and standard deviation error bars, 37H 


\subsubsection{Combine Even and Odd Beams}

The error plots reveal that there may be a slight difference caused by various incidence angles in the $37 \mathrm{GHz}$ channel. Even beams are pointed at an EIA of $58^{\circ}$, while odd beams are angled at $52^{\circ}$. This pattern is evident in the $37 \mathrm{~V} \& H$ channels. Because the error distances for even and odd beams are very similar, they can be analyzed together. This will increase the number of points in our statistical analysis when the boundaries are separated by other criterion such as latitude, land/water or water/land, or angle of intersection.

Based on preliminary analysis, all beam geolocation errors appear to be similarly distributed. Moreover, the mean error distance meets the pointing requirement; however, the standard deviation $( \pm 10 \mathrm{~km})$ of the estimate of the mean error is larger than desired. Further, all eight beams have mean distance errors that are slightly negative. This indicates that the sensor is observing the boundary slightly before the true coastline.

\subsubsection{Boundary Transition Type}

It is important to check that the boundary type does not play a role in the error analysis. It was mentioned earlier, in Chapter 3, that there are two types of transitions we are analyzing, land to water and water to land. The ice boundaries are ignored by setting latitude restrictions. The threshold criterion is an efficient way to eliminate extended boundaries due to an imperfect transition. To validate that there is not a detectable error between error distance associated with land to water and water to land boundaries, the mean and standard deviation will be compared for ascending and descending passes of all channels. The following figures, 4-19 - 24, illustrate the difference in transition type. 


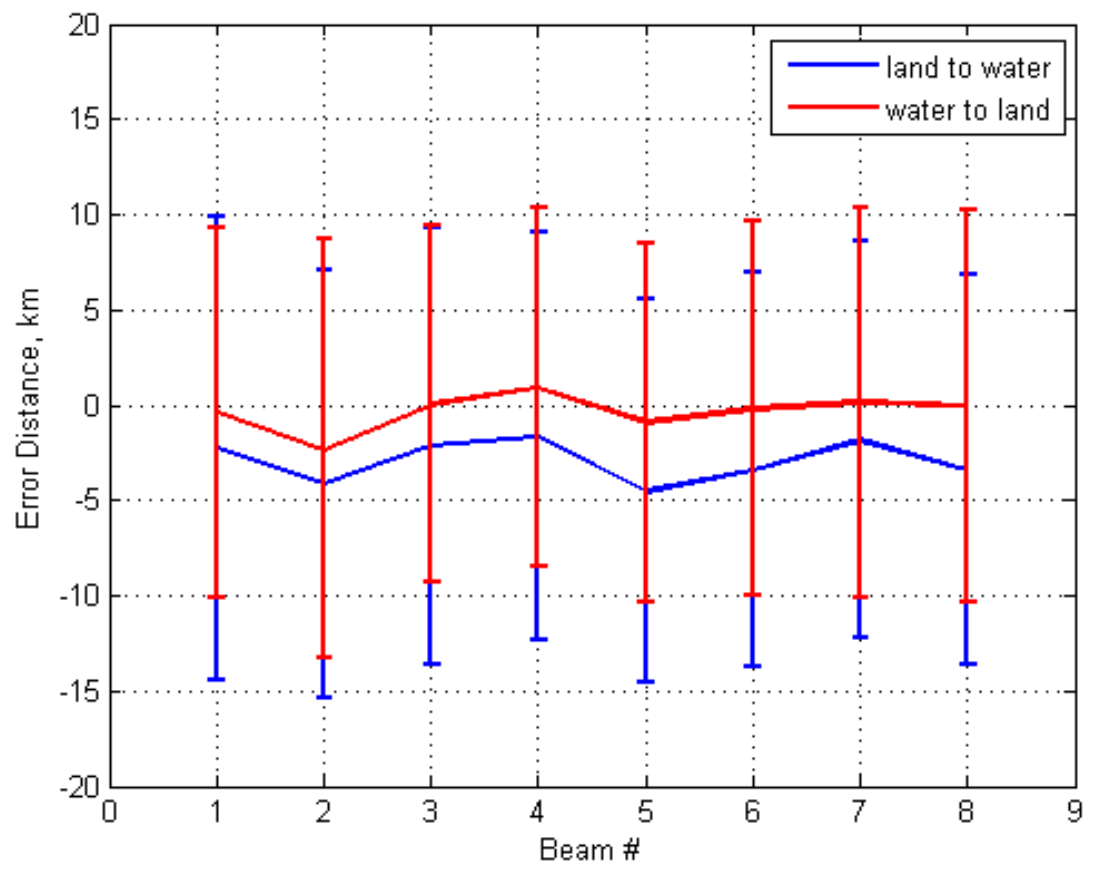

Figure 4-19 23H, ascending transition error

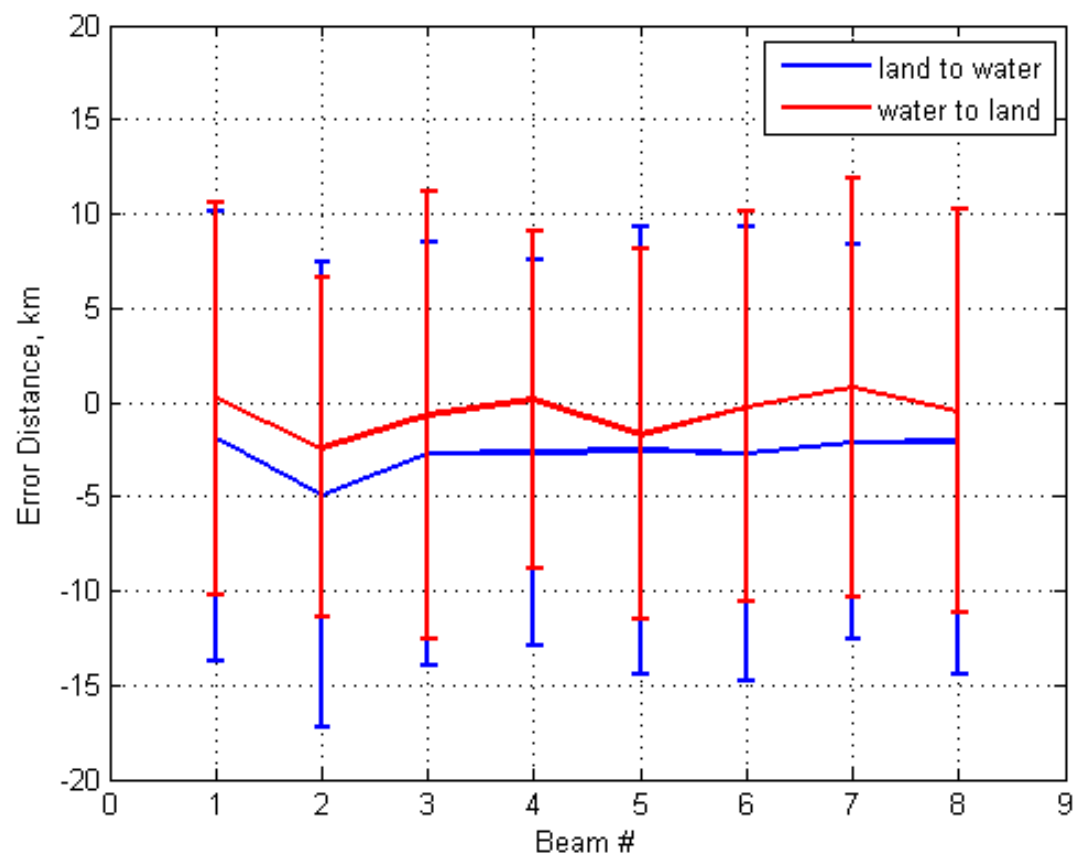

Figure 4-20 23H, descending transition error 


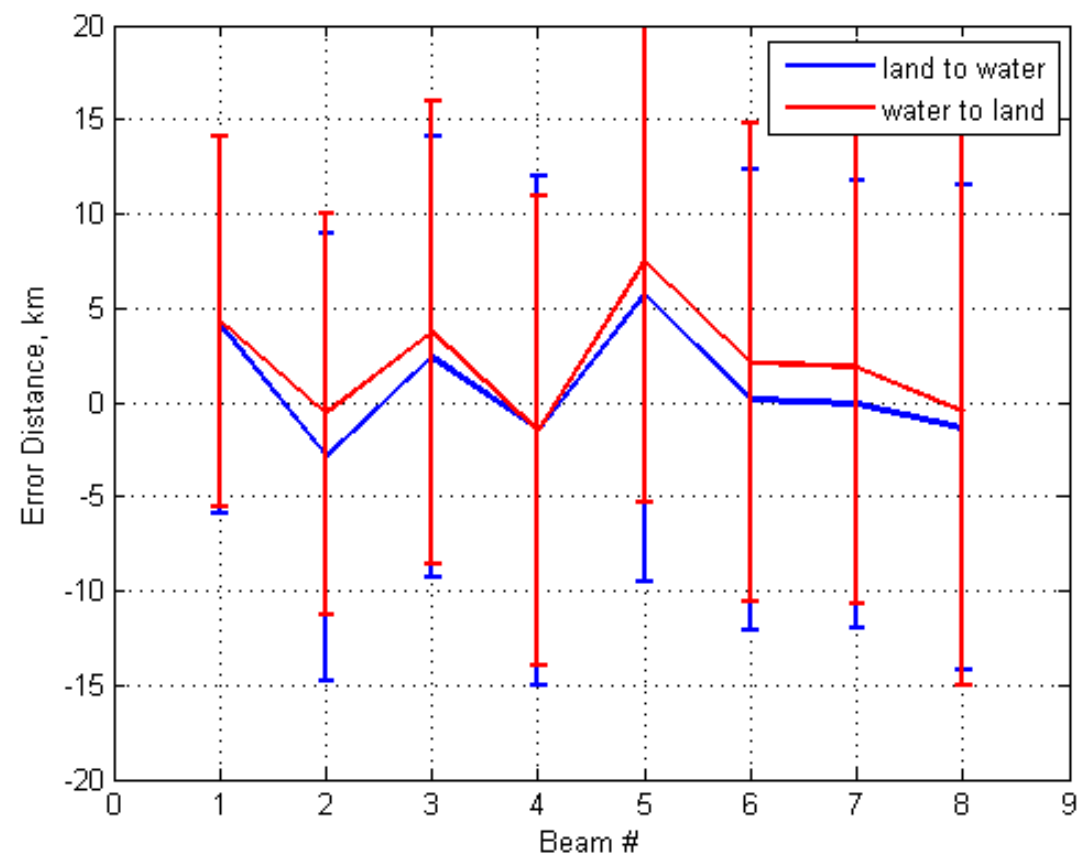

Figure 4-21 37V, ascending transition error

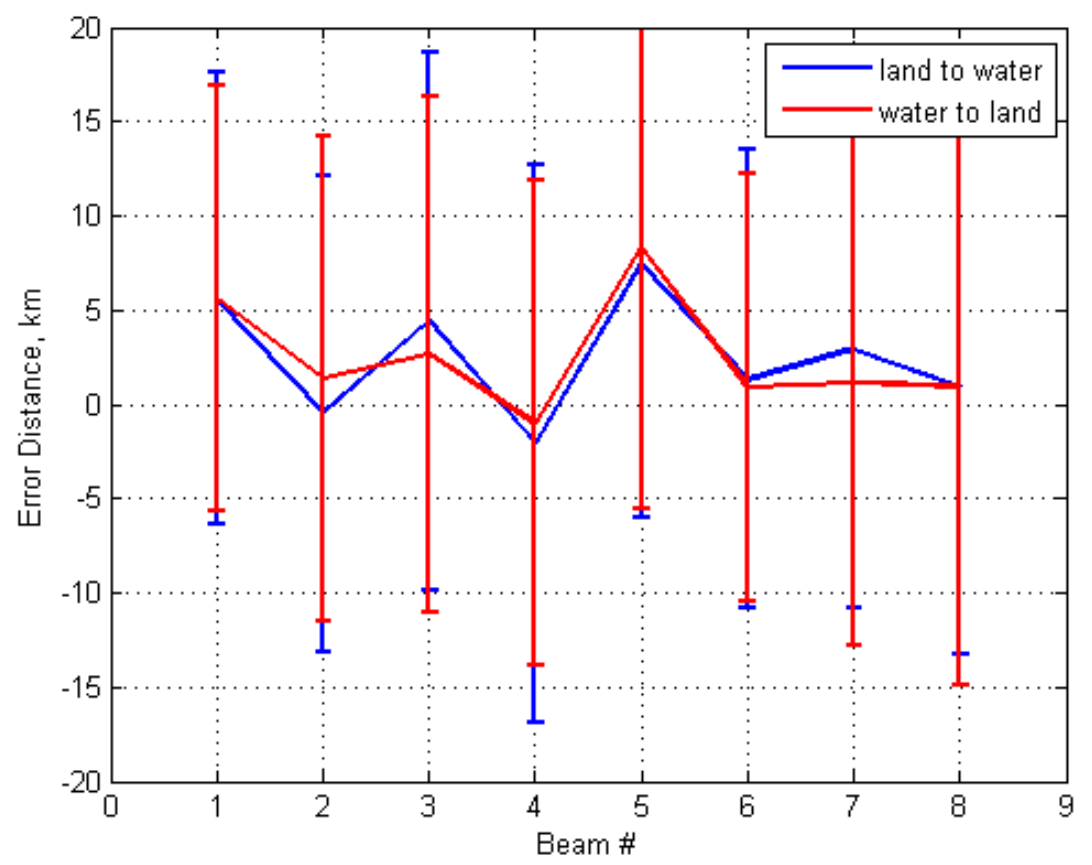

Figure 4-22 37V, descending transition error 


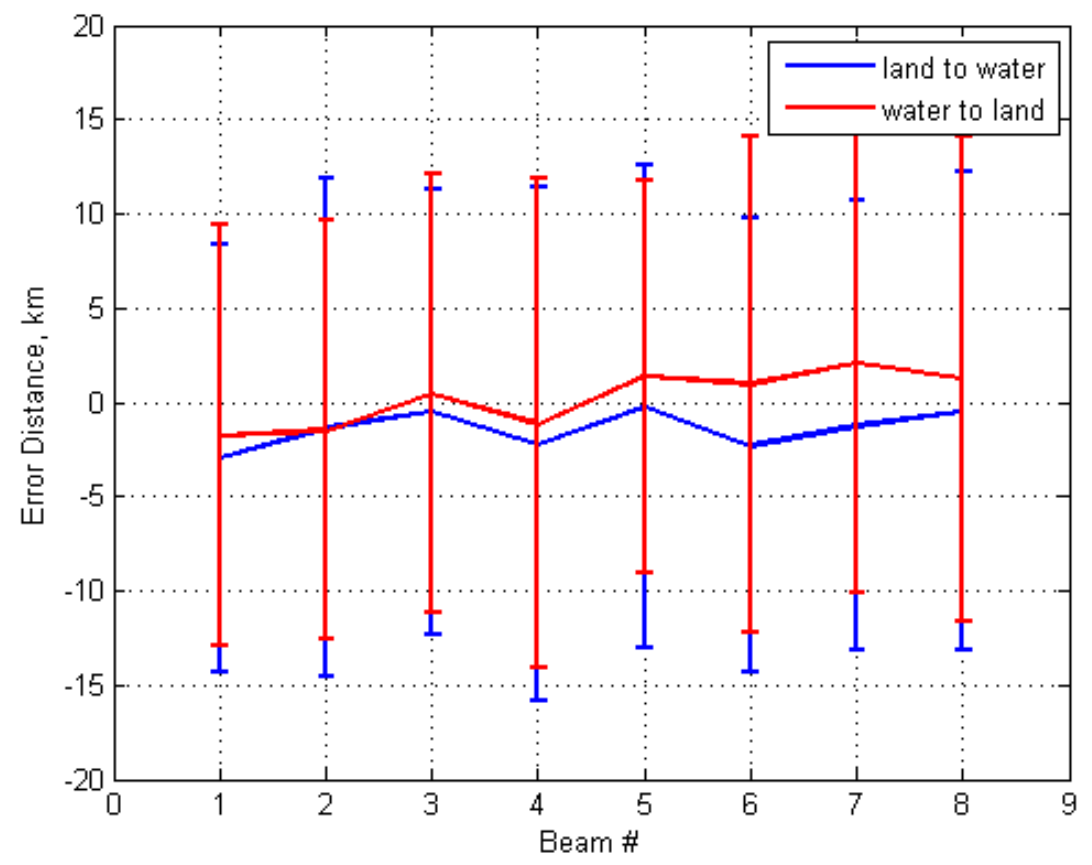

Figure 4-23 37H, ascending transition error

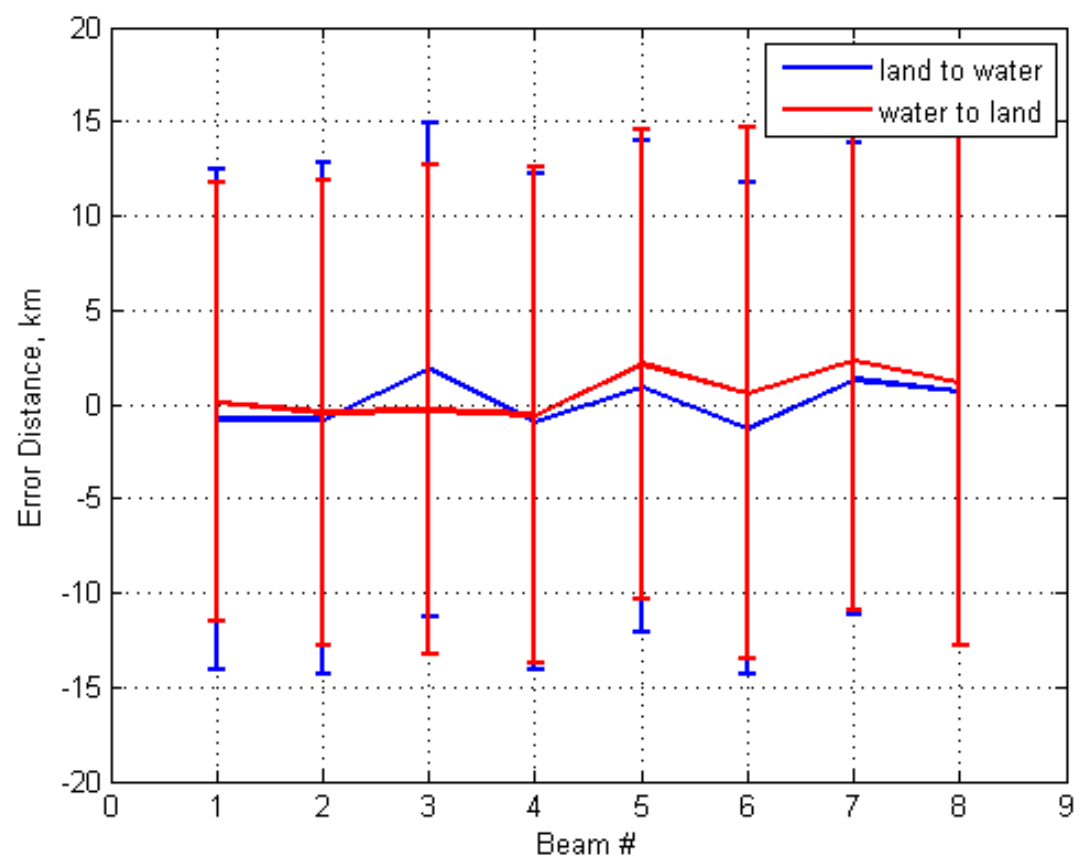

Figure 4-24 37H, descending transition error 
These plots show that there is not a consistent error in all three channels. In fact, in the 37V channel, the error charts lie right on top of one another. If the transition type was causing an error, it would be evident in all channels. This error analysis validated that the type of boundary does not make a significant contribution to the error distance calculation.

\subsubsection{Latitude Dependence}

Yaw is a spacecraft attitude error that can be discovered by comparing the error distance calculations at various latitudes. If a yaw error is not present, the error distance will be offset from 0 , but it will remain near constant at all latitudes. When an error is present, the error distance will vary with latitude. The boundaries were classified into three latitude bins: -40 to $10^{\circ},-10$ to $10^{\circ}$, and 10 to $40^{\circ}$ latitude. This did not distribute the number of points evenly, but that is due to the fact that there are more land/water boundaries in the northern hemisphere. There were still plenty of points in each bin since it has been determined that the even and odd beams can be combined together.

Figures 4-25 - 28 are comparing all three channels, even and odd beams at various latitudes. The colors represent each channel: blue is $23 \mathrm{H}$, red is $37 \mathrm{~V}$ and black is $37 \mathrm{H}$. In the charts, the odd beams have larger offsets, which is to be expected. In the $37 \mathrm{~V}$, odd beams produced much greater error distance values for all even beams. Another thing to note, is that the odd beams are affected more by latitude than the even beams. Even beams' error distances

remain around zero, while odd beams have a change of approximately $5 \mathrm{~km}$ from -25 to $25^{\circ}$ latitude in Fig. 4-26. This is contrary to what is expected. 


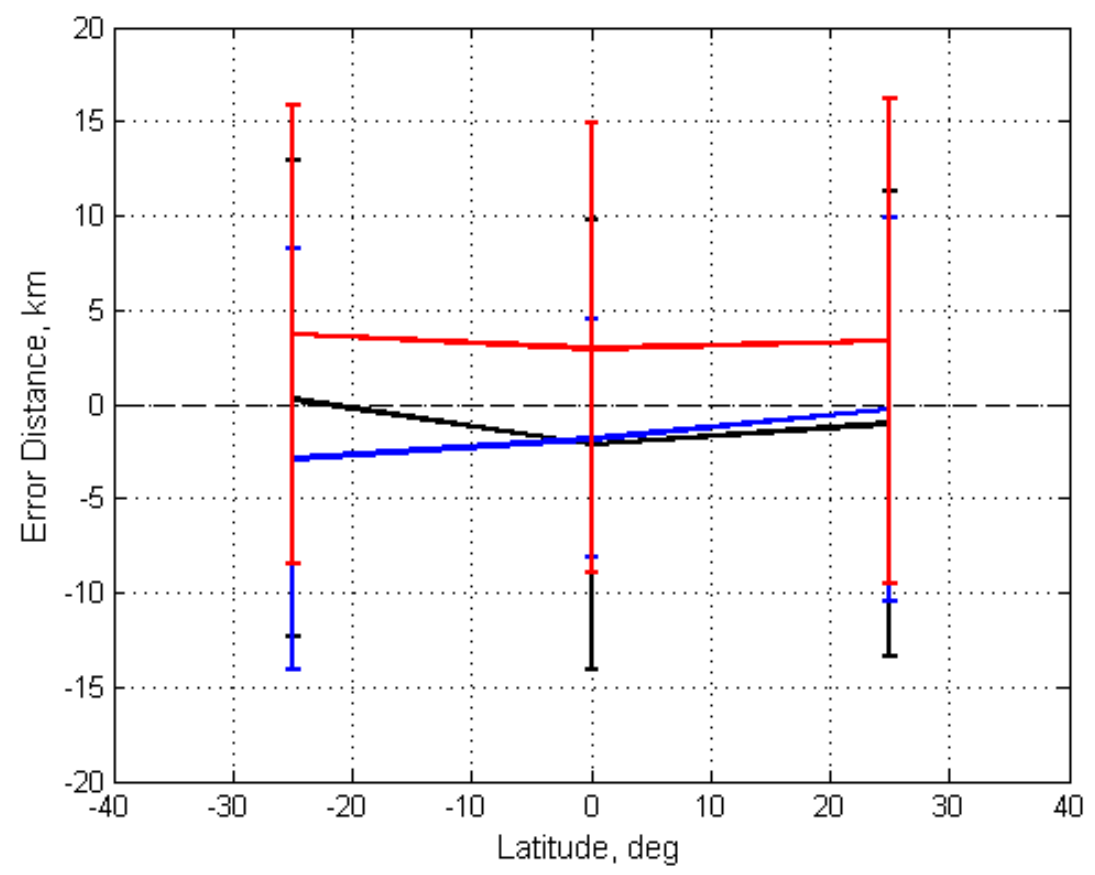

Figure 4-25 Ascending, odd beams

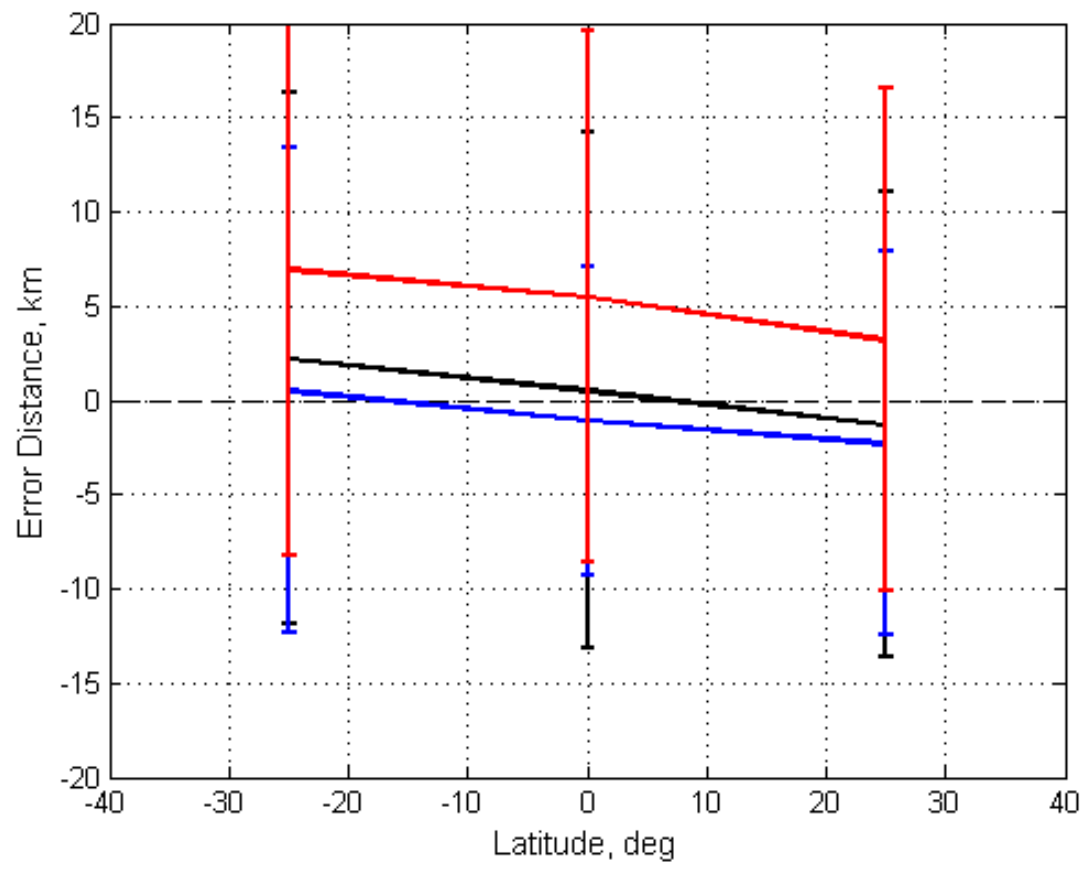

Figure 4-26 Descending odd beams 


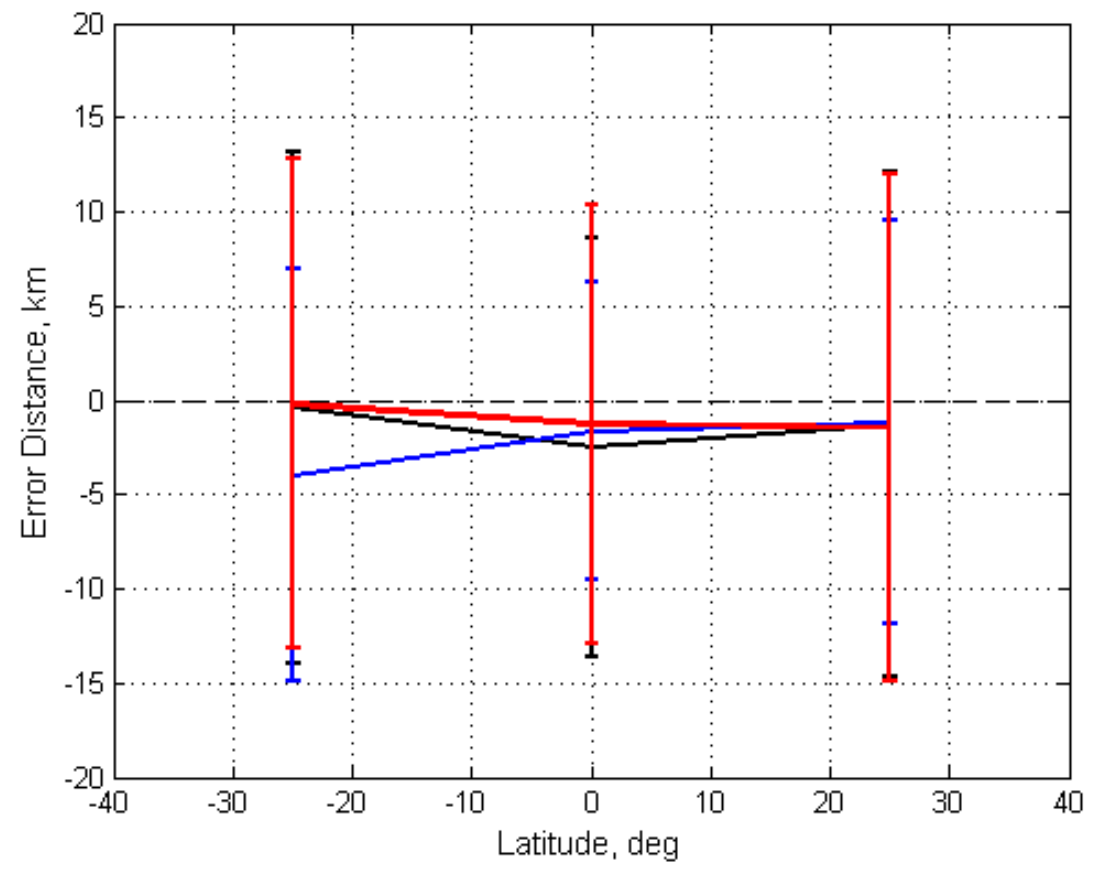

Figure 4-27 Ascending, even beams

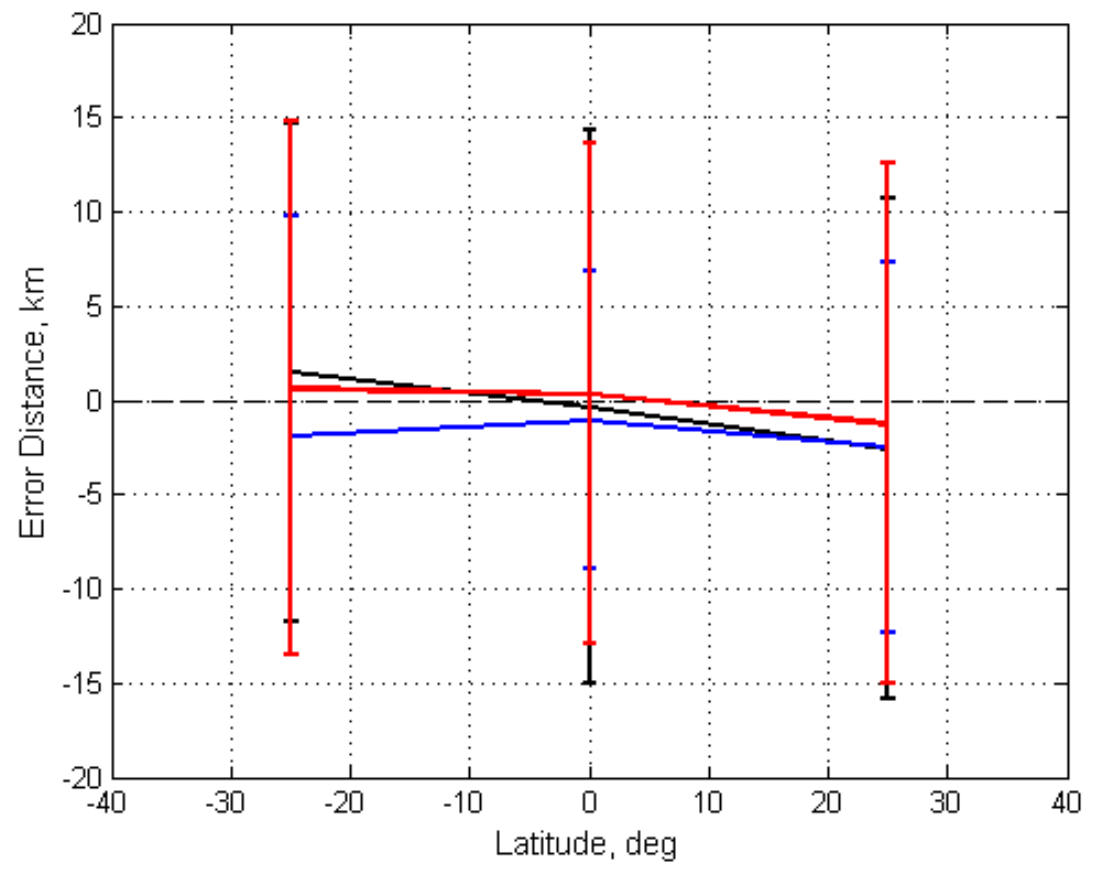

Figure 4-28 Descending, even beams 


\subsubsection{Angle of Intersection}

Characterizing a boundary by its angle of intersection between the satellite trajectory and the coastline is a very useful tool for attitude analysis. This effect is demonstrated in Fig. 4-29. In this image, the satellite motion is to the northwest (ascending pass). Two scenarios are shown: the satellite trajectory is perpendicular to the coast, and the trajectory is parallel to the coast. In the first scenario, pitch can be determined. A positive pitch would cause the beam footprint to shift forward along the path causing the sensor to observe the boundary before it is expected. When near perpendicular boundaries are analyzed, the calculated error distance is exactly caused by a pitch error of the spacecraft or sensor.

Ideally, roll error can be calculated using the same method. However, if the satellite is flying near parallel to a boundary, the Tb slope will be minimal. This intersection method cannot be used by itself to calculate roll, because parallel crossings would not produce a boundary that passes the Tb slope threshold.

Initial results of this testing indicate that the difference in error due to angle of intersection is minimal. Figure 4-30 is a histogram of the angles of intersection for the land/water boundaries of all even beams in the $23 \mathrm{H}$ channel. Notice that there are not many boundaries with intersection angles smaller than 30 degrees or larger than 110 degrees. Again, this is due to the near-parallel effect of $\mathrm{Tb}$ slope. Those angles do not produce boundaries with Tb slopes greater than the threshold criteria. Figure 4-31 is the errorbar chart for the data contained in the histogram. There is very little variation in mean and standard deviation error distance as the angle of intersection changes. 


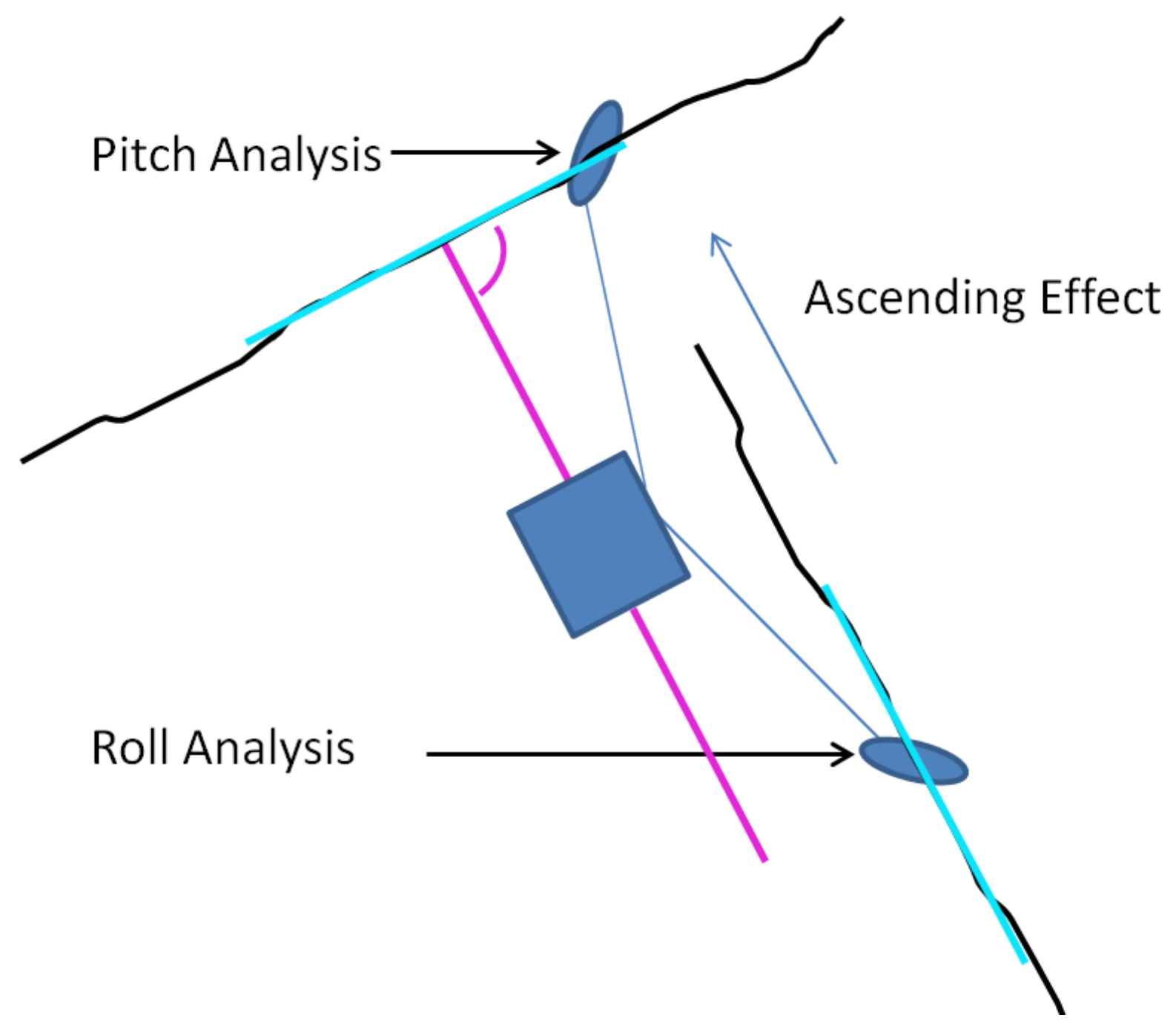

Figure 4-29 Angle of intersection used to determine attitude errors: perpendicular and parallel. 


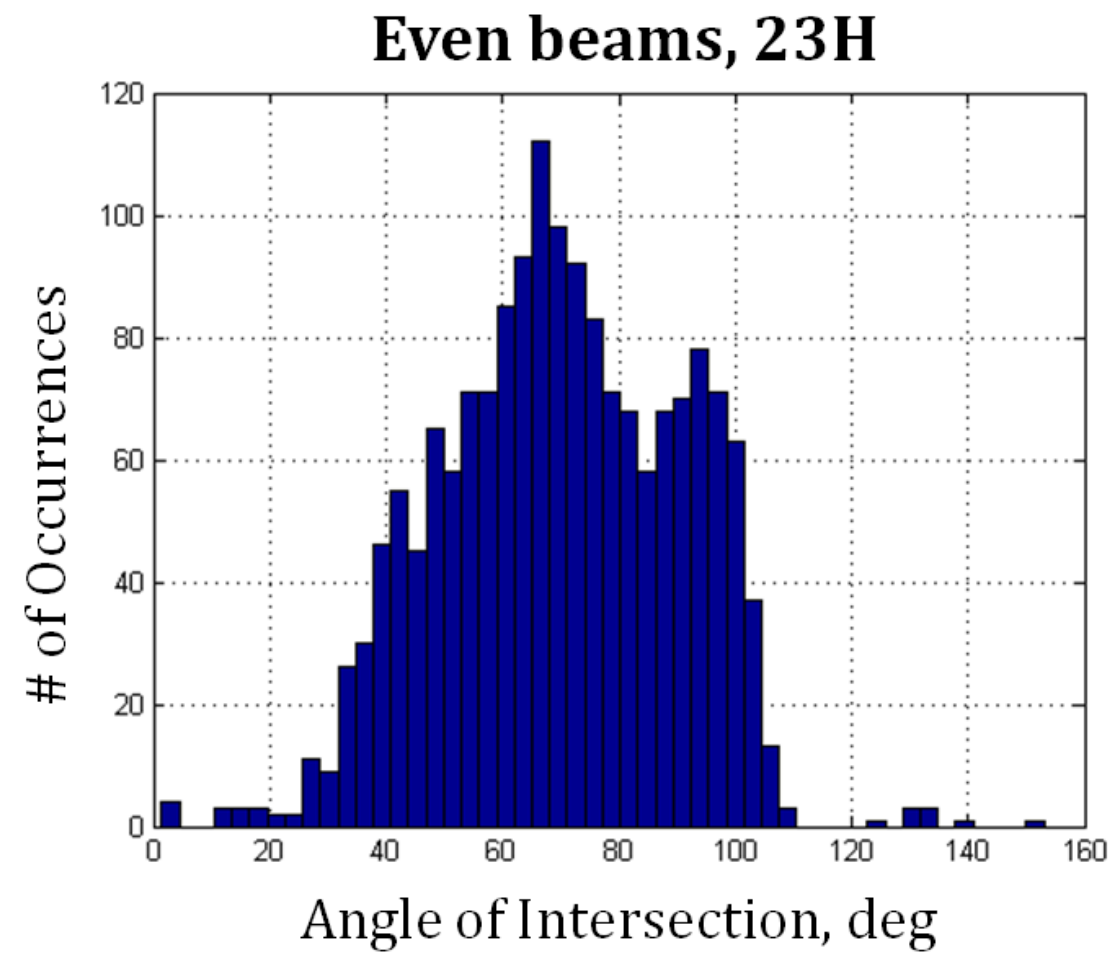

Figure 4-30 Histogram of MWR and coastline angle of intersection for even beams, $23 \mathrm{H}$

Even beams, 23H

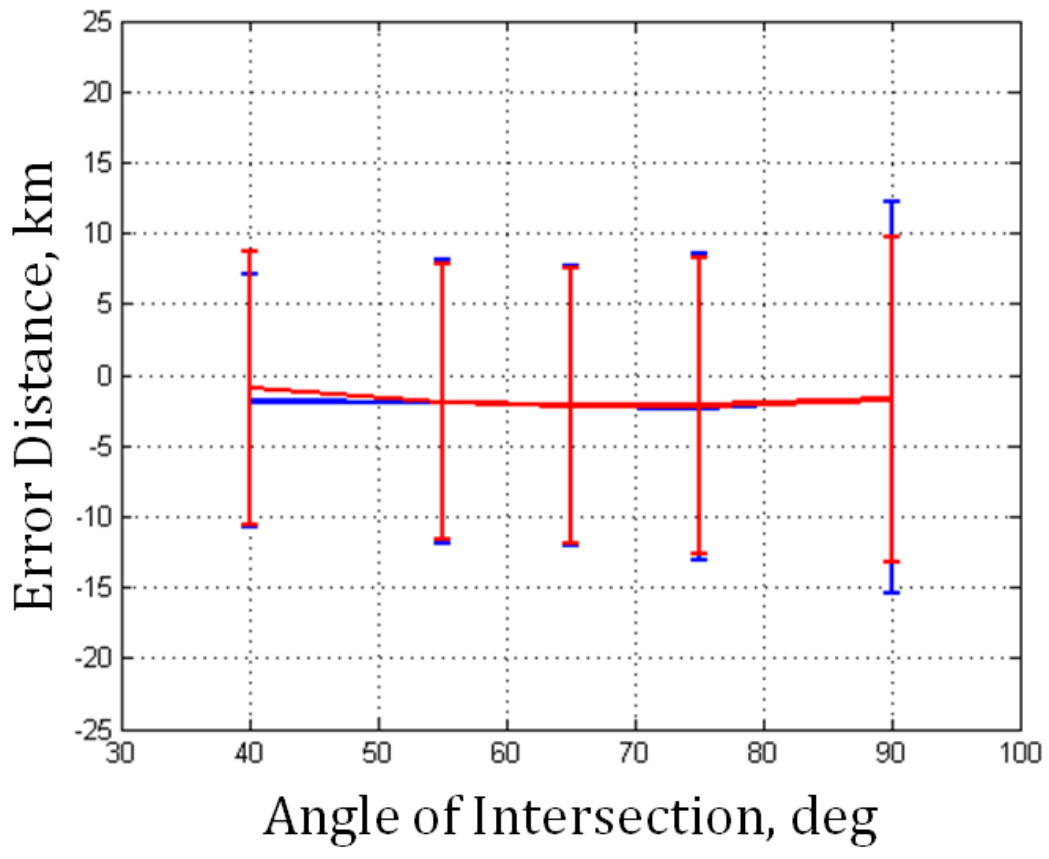

Figure 4-31 Mean and standard deviation error distance by angle of intersection 


\section{CHAPTER 5: CONCLUSIONS AND FUTURE WORK}

\section{$\underline{5.1 \text { Summary }}$}

The geolocation assessment algorithm was implemented successfully providing a detailed description of mispointing error for the Microwave Radiometer on-board Aquarius/SAC-D. All eight beams of the $23.8 \mathrm{GHz}$ horizontal polarization, $36.5 \mathrm{GHz}$ vertical and horizontal polarization channels of MWR have been analyzed by using several different comparison methods.

Initially, an error comparison was conducted separately for all beams in each channel. There showed to be a similar error distance for even and odd beams which was beneficial for further analysis. It is mostly a sanity check that the even and odd beams’ error distances match. The antenna beam pattern would be the main cause for error between those beams. However, it is important that they remain separate since varying incidence angles are affected differently by pitch error.

Then, the error distances due to the type of crossing were compared. Again, this was a sanity check because there should not be a systematic difference between land to water and water to land transitions. This type of error was not observed; therefore, the data sets that we are using contain 'good' boundaries where the transition was spread out over many kilometers. This signifies that the threshold we set for each channel is correct.

An analysis of the latitude dependence was also conducted. Changes in error distance by latitude can indicate that the satellite is not properly yawed at certain locations. Yaw steering implementation cannot be specifically analyzed by this method, because it must take into account 
the forward and aft beam comparison. This is simply a brute force comparison of error distance as a function of latitude.

Finally, and most importantly, angle of intersection analysis was conducted. By separating the boundaries by angle of intersection, we are able to determine which errors are associated with satellite attitude. Boundaries with an angle of intersection of $90^{\circ}$ can reveal pitch errors and angles of $0^{\circ}$ or $180^{\circ}$ can reveal roll errors, as mentioned in Chapter 4.

\section{$\underline{5.2 \text { Conclusions }}$}

This geolocation assessment is a very good technique for assessing satellite attitude errors. It obtains numerous MWR sensor observed boundaries with a high level of accuracy on a large scale. The robust data set, which provided a large number of crossings, was ideal because it allowed multiple separation methods to analyze the data.

Overall, the error distance calculations were small. The largest mean error observed ( $~ 8$ $\mathrm{km}$ ) is much less than the length of one MWR IFOV. It is also less than the $13 \mathrm{~km}$ MWR sampling distance. The standard deviation of most errors is approximately $10 \mathrm{~km}$, which indicates that the majority (>85\%) of the sensor observed boundaries occur less than $15 \mathrm{~km}$ from the true coastline.

Many errors can occur with this geolocation algorithm, as described in Chapter 3, but actions have been taken to account for these errors. For example, using a low resolution coastal map can cause errors rather large errors when calculating the error distance from the observed boundary to the true boundary ( $>5 \mathrm{~km}$ in some cases). Values like this are unacceptable; the 'true' map should have very minimal errors associated with it. This error was mitigated by 
increasing the resolution to a one kilometer resolution map. Other errors such as the sampling distance quantization and boundary transition error have also been minimized.

\subsection{Future Work}

This work is on-going. Calculating the specific attitude errors has proven to be quite cumbersome. Roll and pitch error, in terms of distance on Earth's surface, have been determined with a fairly high accuracy. However, yaw error has been partially analyzed by comparing the error distance with latitude. A full assessment of yaw steering has not been completed. This analysis will need to be performed on a more individual boundary basis between forward and aft beams at all latitudes.

The next task is to determine if this error is consistent in all channels and with the Aquarius footprints. This will help to deduce where the error lies, mounting of MWR sensor, the entire satellite platform, etc. Finally, when the exact satellite attitude errors have been agreed upon, the analysis will be delivered to CONAE. 


\section{LIST OF REFERENCES}

[1] G. Lagerloef, F. R. Colomb, D. LeVine, et al., "The Aquarius/SAC-D Mission,” Oceanography, vol. 21, no. 1, pp. 68-81, Mar. 2008.

[2] S. Farrar, "Preliminary Analysis of the Aquarius/SAC-D Microwave Radiometer (MWR) Antenna Temperature,” presented at IEEE Southeast Conf.,Orlando, FL, 2012.

[3] Juan Cruz Gallo, Daniel Omar Rocca, Linwood Jones and Sayak Biswas, "MWR Calibration, Pre-launch and Post-launch", 6th Aquarius/SAC-D International Science Symposium, Jul. 1921, 2010, Seattle, WA

[4] F. Madero, “MWR Geolocation”, MWR Processor Geometric ATBD, pp. 51-54, Aug. 2011.

[5] Kepler's Laws of Planetary Motion. 2012. Wikipedia, the Free Encyclopedia. Mar. 2012. $<$ http://en.wikipedia.org/wiki/Kepler\%27s_laws_of_planetary_motion>

[6] W. Purdy, P. Gaiser, G. Poe, E. Uliana, T. Meissner, F. Wentz, “Geolocation and Pointing Accuracy Analysis for the WindSat Sensor,” IEEE Trans. Geosci. Remote Sens., vol. 44, no. 3, pp. 496-505, Mar. 2006.

[7] G.A. Poe and R. W. Conway, "A study of the geolocation errors of the Special Sensor Microwave/Imager (SSM/I),” IEEE Trans. Geosci. Remote Sens., vol. 28, no.5, pp. 791-799, Sep. 1990.

[8] S. K. Biswas, "Brightness Temperature Calibration of SAC-D/Aquarius Microwave Radiometer,” Ph.D. defense, Dept. Elect. Eng., Univ. of Central Florida, Orlando, FL, 2011.

[9] S. S. Khan, "Simulation of Brightness Temperatures for the Microwave Radiometer on the Aquarius/SAC-D Mission,” M.S. thesis, Dept. Elect. Eng., Univ. of Central Florida, Orlando, FL, 2009.

[10] F.T. Ulaby, R.K. Moore, A.K. Fung, “Radiometry,” in Microwave Remote Sensing, Norwood: Artech House, 1981, ch. 4, sec. 5.3, pp.204.

[11] J. Skora, "Medicion de Propiedades de Radiacion de Modelo Protoflight de Antena Multihaz para Instrumento Maria en Banda Ka,” MWR chamber data. Sept. 2008.

[12] Personal communications, Remote Sensing Systems, Jan. 2012. 